\title{
Searching for heavy Higgs in supersymmetric final states at the LHC
}

\author{
Amit Adhikary, ${ }^{a}$ Biplob Bhattacherjee, ${ }^{a}$ Rohini M. Godbole, ${ }^{a}$ Najimuddin Khan $^{a}$ \\ and Suchita Kulkarni ${ }^{b}$ \\ ${ }^{a}$ Centre for High Energy Physics, Indian Institute of Science, \\ Bengaluru - 560012, India \\ ${ }^{b}$ Institut für Hochenergiephysik, Österreichische Akademie der Wissenschaften, \\ Nikolsdorfer Gasse 18, 1050 Wien, Austria \\ E-mail: amitadhikary@iisc.ac.in, biplob@iisc.ac.in, rohini@iisc.ac.in, \\ najimuddink@iisc.ac.in, suchita.kulkarni@oeaw.ac.at
}

ABSTRACT: In this work, we analyse and demonstrate possible strategies to explore extended Higgs sector of the Minimal Supersymmetric Standard Model (MSSM). In particular we concentrate on heavy Higgs decays to electroweakinos. We analyse the Higgs to electroweakino decays in the allowed MSSM parameter space after taking into account $13 \mathrm{TeV}$ LHC searches for supersymmetric particles and phenomenological constraints such as flavour physics, Higgs measurements and dark matter constraints. We explore some novel aspects of these Higgs decays. The final states resulting from Higgs to electroweakino decays will have backgrounds arising from the Standard Model as well as direct electroweakino production at the LHC. We demonstrate explicit kinematical differences between Higgs to electroweakino decays and associated backgrounds. Furthermore, we demonstrate for a few specific example points, optimised analysis search strategies at the high luminosity LHC (HL-LHC) run. Finally, we comment on possible search strategies for heavy Higgs decays to exotic final states, where the lightest chargino is long lived and leads to a disappearing track at the LHC.

Keywords: Supersymmetry Phenomenology

ARXIV EPRINT: 2002.07137 


\section{Contents}

1 Introduction 1

2 Benchmark scenarios and their features 3

3 pMSSM random scan $\quad 9$

$\begin{array}{lll}3.1 & \text { Scan results } & 11\end{array}$

$\begin{array}{lll}3.2 & \text { Mono-Z event kinematics } & 14\end{array}$

$\begin{array}{lll}3.3 & \text { LLCP kinematics } & 17\end{array}$

4 Collider analysis $\quad 18$

4.1 The mono-Z final state 24

4.1.1 The $\ell \ell+\mathbb{E}_{T}$ channel $\quad 24$

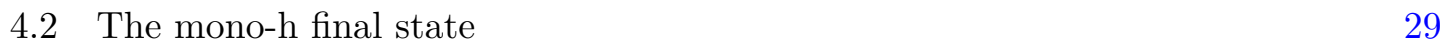

4.2.1 The $b \bar{b}+\mathbb{E}_{T}$ channel 30

4.2.2 The $\gamma \gamma+\mathbb{E}_{T}$ channel 34

$5 \quad$ Long lived charged particle (LLCP) $\quad 38$

5.1 Decay fraction of LLCP at various tracker ranges in the detector 39

5.2 Probing charged Higgs via LLCP signature 42

5.2.1 $\ell \ell+$ LLCP 43

$\begin{array}{lll}5.2 .2 & b \bar{b}+\mathrm{LLCP} & 44\end{array}$

$\begin{array}{lll}5.2 .3 & \gamma \gamma+\text { LLCP } & 45\end{array}$

6 Conclusion 46

$\begin{array}{ll}\text { A Random scan results } & 47\end{array}$

$\begin{array}{ll}\text { B Parton level kinematics } & 49\end{array}$

C Summarising the cross sections and generator level cuts for the SM $\begin{array}{ll}\text { backgrounds } & \mathbf{5 0}\end{array}$

\section{Introduction}

In the search for extensions of the Standard Model, the pursuit of extended Higgs sector remains an important avenue to determine whether the Standard Model (SM) Higgs is the only elementary scalar or is a part of family. Such heavy Higgs is being searched for at the LHC via its decays to the SM final states. The Minimal Supersymmetric Standard Model (MSSM) is an example where such an extended Higgs sector necessarily arises and contains two Higgs doublets [1-4]. The MSSM Higgs sector at tree level can be described completely by only two parameters, viz. the ratio of the vacuum expectation value (vev) of the two Higgs doublets, $\tan \beta$ and the pseudoscalar Higgs mass, $M_{A}$. There are five Higgses 
in this model as compared to only the one in the SM. These are two neutral scalar Higgses $(\mathrm{h}, \mathrm{H})$, one neutral pseudoscalar Higgs $(\mathrm{A})$ and two charged Higgses $\left(H^{ \pm}\right)$. The neutral heavy Higgs boson can be produced at the LHC via the gluon-gluon fusion ( $\mathrm{ggH})$ or the bottom quark annihilation (bbH). Production of heavy Higgs boson in association with a vector boson and via the vector boson fusion channels are suppressed due to alignment limit implied by observed properties of the SM Higgs. The relative strength of the $\mathrm{ggH}$ and $\mathrm{bbH}$ production modes is determined by $\tan \beta$. A similar consideration also determines the dominant decay mode of the heavy Higgs. Note that $W W / Z Z$ final states are suppressed due to alignment limit. At large $\tan \beta$, heavy Higgs coupling to down-type quark and leptons become important. Therefore, in this case, the $b \bar{b}$ fusion production rate $(b \bar{b} \rightarrow$ $H / A$ ) dominates over the gluon fusion mode and the branching ratio for $H / A \rightarrow \tau^{+} \tau^{-}$ becomes large. Thus the channel, $b \bar{b} \rightarrow H / A \rightarrow \tau^{+} \tau^{-}$probes low $M_{A}$ and high $\tan \beta$ parameter space [5]. These probes are further complemented with $g g \rightarrow H / A \rightarrow h h$ [6-8] and $g g \rightarrow H / A \rightarrow t \bar{t}[9,10]$ searches in the low $\tan \beta$ parameter space.

Despite negative results from these searches, a large part of heavy Higgs parameter space remains allowed, particularly in the intermediate $\tan \beta$ regime. This intermediate $\tan \beta$ regime is specially difficult to probe via the Standard Model (SM) final states as in this case the heavy Higgs branching ratio to SM final states is overtaken by that to supersymmetric final states, if kinematically allowed. Among supersymmetric particles, the most interesting sector is the electreoweakino sector of the MSSM. Due to comparatively weaker LHC limits, the heavy Higgs to decays into these final states are still possible. The MSSM electroweakino sector consists of four neutralinos and two charginos. The neutrali$\operatorname{nos}\left(\widetilde{\chi}_{1}^{0}, \widetilde{\chi}_{2}^{0}, \widetilde{\chi}_{3}^{0}, \widetilde{\chi}_{4}^{0}\right)$ are mixtures of the gauginos i.e. bino $\left(\tilde{B}^{0}\right)$, wino $\left(\tilde{W}^{0}\right)$ and higgsinos i.e. $\tilde{H}_{u}^{0}, \tilde{H}_{d}^{0}$. Similarly the charged components of the gaugino i.e. $\tilde{W}^{ \pm}$and higgsino i.e. $\tilde{H}_{u}^{+}, \tilde{H}_{d}^{-}$mix to form 2 chargino mass eigenstates $\left(\widetilde{\chi}_{1}^{ \pm}, \widetilde{\chi}_{2}^{ \pm}\right)$. We refer to all of them collectively as electroweakinos. The heavy Higgs can therefore decay into any combination of these four neutralinos or two charginos depending on phase space and couplings. This gives rise to multiple heavy Higgs decay modes. We collectively label such decays of heavy Higgs to supersymmetric (susy) final state as Higgs to susy decays throughout this work.

Complementary to the searches in the decays of heavy Higgs (see for example ref. [11]), multiple LHC searches for direct electroweakino production exist. These target electroweakino production via SM mediators i.e. $Z, W, \gamma$ and decays into SM final states in association with missing energy $\left(\mathbb{E}_{T}\right)$. Such production modes however require off-shell SM mediators as the collider searches have constrained the electroweakino masses above $100 \mathrm{GeV}$ over a large region of parameter space. Production of electroweakinos via heavy Higgs decays on the other hand can target on-shell heavy Higgs as a mediator, yielding distinct kinematics in the final states. This presents another opportunity to search for extended Higgs sector beyond the SM final states discussed before. To exploit the kinematic features and suggest further search strategies for heavy Higgs sector is the main aim of our work.

There have been several studies on the phenomenological aspects of heavy Higgs decays to electroweakinos [12-31]. Many of these analyses demonstrated the importance of mono-X (X $\left.=j, W^{ \pm}, h, Z, \gamma\right)$ final states while exploring heavy Higgs to susy decays, and 
demonstrated the LHC potential to do so. In particular, a recent study [27], demonstrates the reach of HL-LHC for heavy Higgs decays to susy particles in dilepton plus missing energy final state within the MSSM. This study uses the so called clustered transverse mass $m_{C T}$ variable for discrimination between the Higgs to susy signal and the SM backgrounds. It considers only ggH production mode and finally, it does not include direct production of susy backgrounds which also leads to mono-X final states. Going beyond these previous studies, in this work we add the backgrounds arising from direct susy production, consider ggH and bbH Higgs production modes separately and demonstrate the HL-LHC potential to probe Higgs to susy decays in multiple final states.

We employ a strategy similar to the one considered by a recent CMS search [32]. The principle difference between our studies and that of the search is that we target the ggH and bbH production modes separately. We furthermore study the effects of additional b-jets in signal distributions. We work in the framework of specific susy models, which are not considered in the CMS analysis. It will none-the-less be interesting to recast the CMS search to understand the reach for models considered in this work. This is beyond the scope of the work and we leave it for future studies.

With respect to signal over background optimisation, a particularly interesting situation arises while analysing Higgs decays to chargino. In general within the MSSM parameter space, the lightest chargino can be long lived [33, 34], particularly if it is wino-like. Should the heavy Higgs branching ratio to chargino be large, it can lead to heavy stable charged particles or disappearing tracks at the LHC, which have very little background from other sources in detector. In the final part of our work, we elaborate such possible decay modes and suggest a few strategies for searches.

The plan of the paper is as follows: we investigate a few benchmark scenarios and discuss Higgs to susy cross sections in section 2. In section 3, we discuss the numerical setup of our 19 dimensional MSSM parameter space scan and demonstrate the cross sections for mono-X final states, we also explore salient kinematical differences between signal and background distributions. From here onwards we specifically look at the impact of resonance mediated susy production on the event kinematics, we explicitly demonstrate the impact of presence of a resonance. In section 4 we propose for a few benchmark points, optimised set of cuts leading to several different significances. We furthermore present a benchmark study of LLP in section 5. Finally in section 6, we conclude.

\section{Benchmark scenarios and their features}

The relative hierarchy of the higgsino-gaugino mass parameters affect the gaugino-higgsino content of the electroweakino mass eigenstates. The heavy higgs decays to electroweakinos, if kinematically allowed, are enhanced if both the gaugino and higgsino content are sizable, as this maxmimises the couplings of the heavy Higgs with the electroweakinos. The same hierarchy also affects the direct production of electroweakinos due to its effects on their couplings to the SM particles as well as their masses. We comment on the relative importance of the two modes, one where electroweakinos are produced via heavy Higgs decays and the other direct electroweakino production. To illustrate possible Higgs to susy 
decay modes and resulting final state at the LHC, in this section we consider three different gaugino-higgsino mass hierarchies. These hierarchies are responsible for generating either bino-higgsino or wino-higgssino-like light electroweakinos. We also compare and contrast this with direct production of electroweakinos via SM mediators.

To illustrate this case by case, we select a few benchmark scenario in the MSSM parameter space and discuss some salient features of the Higgs and electroweakino sector. No experimental constraints are applied at this point for benchmark choices. We have however taken care to keep the lightest Higgs boson mass within $122-128 \mathrm{GeV}$ [35]. An investigation of allowed parameter space by including all experimental constraints will be discussed in section 3. Here, we use Suspect2 [36] to generate the mass spectrum of susy particles. The MSSM parameters are chosen as follows,

$$
\begin{aligned}
& M_{A}=1 \mathrm{TeV}, 4<\tan \beta<20, M_{3}=5 \mathrm{TeV}, A_{t}=-5 \mathrm{TeV}, \\
& A_{e, \mu, \tau, u, d, c, s, b}=0, M_{\tilde{e}_{L}, \tilde{\mu}_{L}, \tilde{\tau}_{L}, \tilde{e}_{R}, \tilde{\mu}_{R}, \tilde{\tau}_{R}}=5 \mathrm{TeV}, \\
& M_{\tilde{Q}_{1_{L}}, \tilde{Q}_{2_{L}}, \tilde{Q}_{3_{L}}}=5 \mathrm{TeV}, M_{\tilde{u}_{R}, \tilde{d}_{R}, \tilde{c}_{R}, \tilde{s}_{R}, \tilde{t}_{R}, \tilde{b}_{R}}=5 \mathrm{TeV} .
\end{aligned}
$$

Keeping these parameters fixed, we change the gaugino and higgsino mass parameters which results in different possible scenarios, as we discuss below. We would like to mention here that the large values of $A_{t}$ parameter may give rise to charge and colour breaking minima (see for example ref. [37]). Of course this is taken care of in the spectrum generator Suspect2.

Case-1. In this scenario, we fix the wino mass parameter $M_{2}$ at $1500 \mathrm{GeV}$ and vary the higgsino and bino masses. This results in a mixed bino-higgsino like scenario.

Case-1a. The higgsino and bino mass parameters are chosen as $\mu=450 \mathrm{GeV}$ and $M_{1}=$ $370 \mathrm{GeV}$. Therefore, the LSP $\left(\widetilde{\chi}_{1}^{0}\right)$ becomes bino-higgsino mixture (bino fraction $89.72 \%$, higgsino fraction $10.26 \%$ ), the 2 nd lightest neutralino $\left(\widetilde{\chi}_{2}^{0}\right)$ is higgsino-like (higgsino fraction $99.83 \%$ ) and the $\widetilde{\chi}_{3}^{0}$ is mixed state of bino and higgsino (bino fraction $10.16 \%$ and higgsino fraction $89.56 \%$ ). In figure 1 we plot the resulting branching ratios as a function of $\tan \beta$. For phase space reasons, the heavy Higgs decays to susy particle always involves one $\widetilde{\chi}_{1}^{0}$ while the other can be $\widetilde{\chi}_{2}^{0} / \widetilde{\chi}_{3}^{0}$. The $H \rightarrow \widetilde{\chi}_{1}^{0} \widetilde{\chi}_{2}^{0}$ and $A \rightarrow \widetilde{\chi}_{1}^{0} \widetilde{\chi}_{3}^{0}$ branching fractions can be as large as $\sim 19 \%$ and $\sim 13 \%$ respectively, depending on the value of $\tan \beta$. Owing to the large bino-fraction, the branching ratio for the $\widetilde{\chi}_{1}^{0}$ pair production can be at most $\sim 2 \%$ (5\%) from $\mathrm{H}(\mathrm{A})$ decay.

Direct production of such neutral electroweakinos is however suppressed as neutralino coupling to $\mathrm{Z}$ requires a purely higgsino like nature, the only available channel here being neutralino production via SM Higgs.

These electroweakinos further decay to $\widetilde{\chi}_{1}^{0}$ in association with a SM final state. The $\widetilde{\chi}_{2}^{0}$ and $\widetilde{\chi}_{3}^{0}$ decay via a $Z$ boson with a $100 \%$ branching ratio due to available phase space and coupling structure. This gives rise to mono- $Z+\mathbb{E}_{T}$ signature at the LHC.

Along with the decays of heavy Higgs to electroweakino final states, it is also interesting to note that the heavy Higgs itself can be produced in cascade decays of heavier electroweakinos. In the benchmark scenario considered, the heaviest neutralino, $\widetilde{\chi}_{4}^{0}$ being 

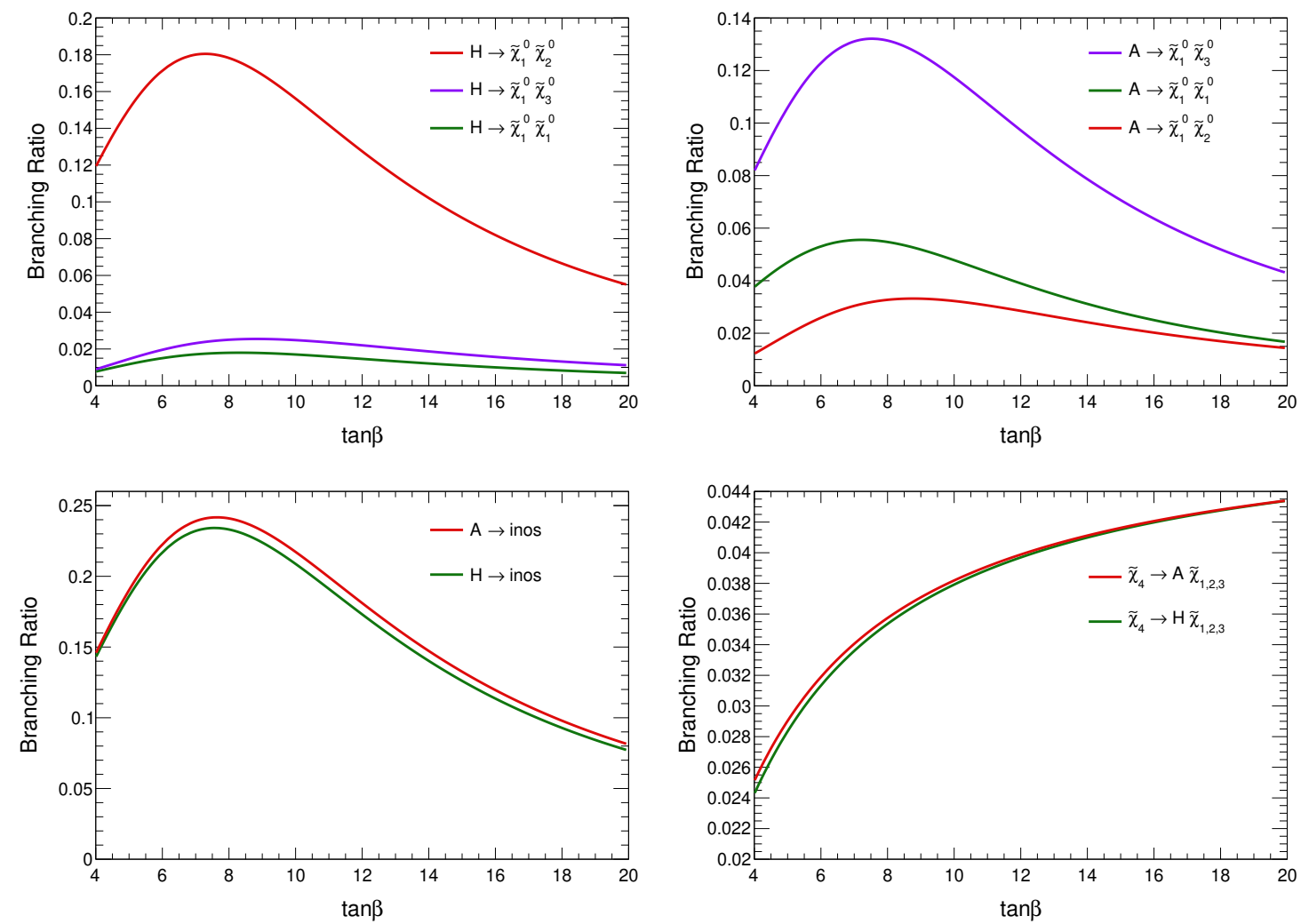

Figure 1. Dominant branching ratios for heavy CP-even and CP-odd Higgs decays to individual electroweakino modes (top panel), the total branching ratio for two heavy Higgs (bottom left), branching ratio of heaviest neutralino decays to heavy Higgs (bottom right) for the case-1a. The wino, bino, higgsino mass parameters $M_{2}, M_{1}, \mu$ are fixed at 1500,450 and $370 \mathrm{GeV}$ and $\tan \beta$ is varied.

heavier than the heavy Higgses, $(\mathrm{H} / \mathrm{A})$ can decay via $\widetilde{\chi}_{4}^{0} \rightarrow(H / A)+\left(\widetilde{\chi}_{1}^{0} / \widetilde{\chi}_{2,3}^{0}\right)$ final state. This branching ratio can reach up to $\sim 4 \%$ in this scenario. This process is important because if this branching is significant then this can contribute to the production of heavy Higgs. At the same time, current limits on heavy Higgs mass requires the progenator electroweakinos to be heavy thus limiting the reach of LHC for such processes. These processes might nonetheless be interesting at future $100 \mathrm{TeV}$ colliders. A detailed investigation is however beyond the scope of this work and we leave it for future considerations.

Case-1b. In this scenario, the higgsino mass parameter is fixed at $\mu=450 \mathrm{GeV}$ and the bino mass parameter is chosen to be $M_{1}=300 \mathrm{GeV}$. Hence like in the previous case, gaugino and higgsino composition are similar except $\widetilde{\chi}_{1}^{0}$ becomes bino-like (bino fraction $95.80 \%$ ). The main difference in this scenario as compared to the previous one is the difference between $M_{1}$ and $\mu$ mass parameter, which is more than the SM Higgs mass. We display the heavy Higgs to electroweakino branching ratios as a function of $\tan \beta$ in figure 2. The heavier neutralinos viz. $\widetilde{\chi}_{2}^{0}$ and $\widetilde{\chi}_{3}^{0}$ can also decay to the LSP via SM Higgs boson. Since $\widetilde{\chi}_{2}^{0}$ is higgsino-like, it dominantly decays to $\widetilde{\chi}_{2}^{0} \rightarrow \widetilde{\chi}_{1}^{0}+Z(\mathrm{BR} \sim 98.17 \%)$ which will give rise to the mono- $Z+\mathbb{E}_{T}$ final state, however since $\widetilde{\chi}_{3}^{0}$ is admixture of bino 

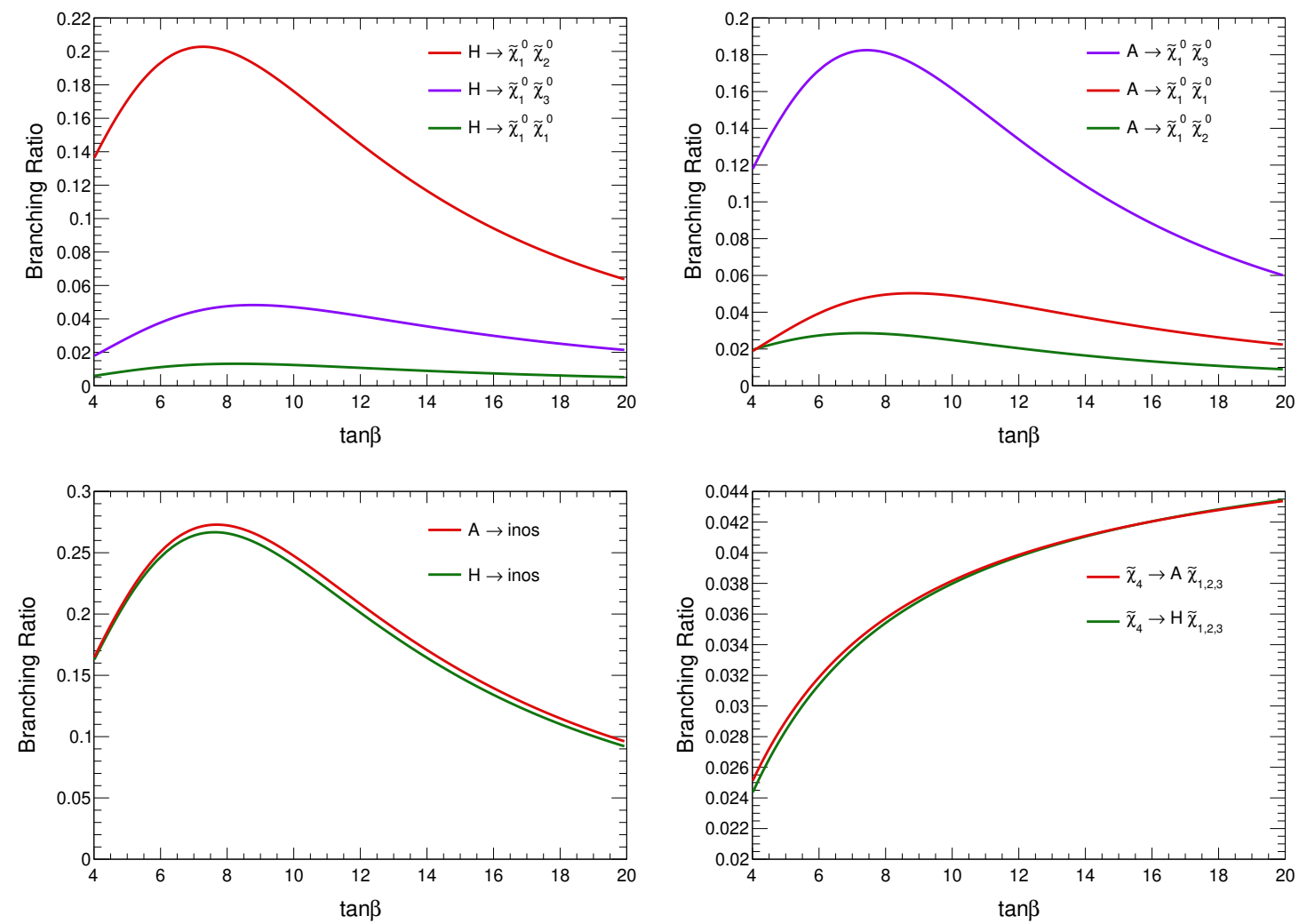

Figure 2. Dominant branching ratios for heavy CP-even and CP-odd Higgs decays to individual electroweakino modes (top panel), the total branching ratio for two heavy Higgs (bottom left), branching ratio of heaviest neutralino decays to heavy Higgs (bottom right) for the case- $1 \mathrm{~b}$. The wino, bino, higgsino mass parameters $M_{2}, M_{1}, \mu$ are fixed at 1500,300 and $450 \mathrm{GeV}$ and $\tan \beta$ is varied.

and higgsino state it dominantly decays to $\tilde{\chi}_{3}^{0} \rightarrow \tilde{\chi}_{1}^{0}+h(\mathrm{BR} \sim 93.98 \%)$. This can lead to a mono-h $+\mathbb{E}_{T}$ signature at the collider.

Case-1c. In the final variation of case-1, we change the bino mass parameter to even lower value, $M_{1}=100 \mathrm{GeV}$. Here we get a bino-like LSP (bino fraction $98.73 \%$ ) and two higgsino state, viz. $\widetilde{\chi}_{2}^{0}, \widetilde{\chi}_{3}^{0}$ (higgsino fraction $98.53 \%, 99.80 \%$ respectively). As the neutralinos in this case are pure states as compared to the cases discussed previously, direct production of pure higgsino state is now possible. The following decay processes can give rise to mono-X final state topologies, viz.

$$
\begin{array}{lll}
p p \rightarrow \widetilde{\chi}_{2}^{0} \widetilde{\chi}_{2}^{0}, & \tilde{\chi}_{2}^{0} \rightarrow \widetilde{\chi}_{1}^{0}+h \\
p p \rightarrow \widetilde{\chi}_{2}^{0} \widetilde{\chi}_{3}^{0}, & \tilde{\chi}_{2}^{0} \rightarrow \widetilde{\chi}_{1}^{0}+h, \quad \widetilde{\chi}_{3}^{0} \rightarrow \widetilde{\chi}_{1}^{0}+Z \\
p p \rightarrow \widetilde{\chi}_{3}^{0} \widetilde{\chi}_{3}^{0}, & \tilde{\chi}_{3}^{0} \rightarrow \widetilde{\chi}_{1}^{0}+Z .
\end{array}
$$

Here, the $\widetilde{\chi}_{2}^{0}, \widetilde{\chi}_{3}^{0}$ can decay to $\widetilde{\chi}_{1}^{0}$ with rate, $B R\left(\widetilde{\chi}_{2}^{0} \rightarrow \widetilde{\chi}_{1}^{0} h\right) \sim 85.93 \%$ and $B R\left(\widetilde{\chi}_{3}^{0} \rightarrow\right.$ $\left.\widetilde{\chi}_{1}^{0} Z\right) \sim 88.13 \%$. The branching ratios of heavy Higgs to electroweakinos are shown in 

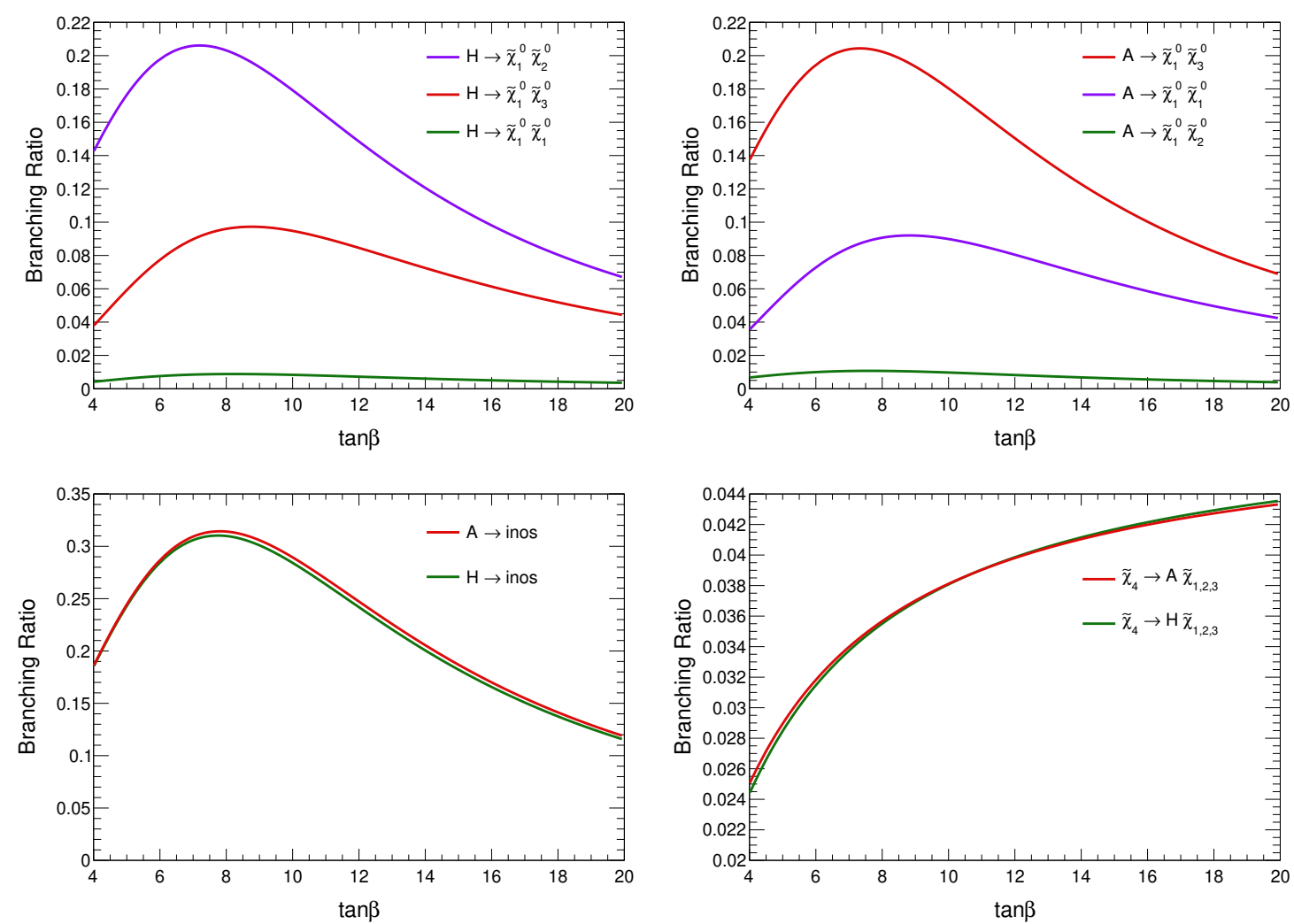

Figure 3. Dominant branching ratios for heavy CP-even and CP-odd Higgs decays to individual electroweakino modes (top panel), the total branching ratio for two heavy Higgs (bottom left), branching ratio of heaviest neutralino decays to heavy Higgs (bottom right) for the case-1c. The wino, bino, higgsino mass parameters $M_{2}, M_{1}, \mu$ are fixed at 1500,100 and $450 \mathrm{GeV}$ and $\tan \beta$ is varied.

figure 3. The generic features for this scenario remain the same as in cast-1b, however in this hierarchy, the $\widetilde{\chi}_{2}^{0}$ decays to $\mathrm{h}$ while the $\widetilde{\chi}_{3}^{0}$ decays to $\mathrm{Z}$ final state.

Case-2. As opposed to previous benchmark where we considered a mixed bino-higgsino benchmark, we now discuss the phenomenological properties of a mixed higgsino-gaugino scenario. This is acquired by considering near degenerate $M_{1}, M_{2}, \mu$. We select the mass parameters as $M_{1}=300 \mathrm{GeV}, \mu=350 \mathrm{GeV}$ and $M_{2}=400 \mathrm{GeV}$, hence resulting in electroweakinos which are mixed state of both the gaugino and higgsino components. As we fix heavy Higgs $(\mathrm{H} / \mathrm{A})$ mass at $1 \mathrm{TeV}$, it can decay to any neutralino/chargino pair. The dominant branching ratios are shown in figure 4 . The heavy Higgs dominantly decays to electroweakinos with maximum branching ratio of $80 \%$ depending on the $\tan \beta$ which is an advantage for searching for heavy Higgs via resonant electroweakino production. It should also be noted that because of the degenerate soft mass parameters, direct production of pure higgsino or gaugino state is highly suppressed. This scenario therefore shows the following unique final state properties. The $\widetilde{\chi}_{2}^{0}$ and $\widetilde{\chi}_{3}^{0}$ can decay to the LSP via 2-body decay as well as 3-body decay. The 3-body decays with appreciable branching ratio are 
the following.

$$
\begin{aligned}
& \widetilde{\chi}_{2}^{0}\left(\widetilde{\chi}_{3}^{0}\right) \rightarrow \widetilde{\chi}_{1}^{0}+\ell^{+}+\ell^{-}(B R \sim 6.15(9.84) \%) \\
& \widetilde{\chi}_{2}^{0}\left(\widetilde{\chi}_{3}^{0}\right) \rightarrow \widetilde{\chi}_{1}^{0}+q+\bar{q}(B R \sim 40.47(64.52) \%) \\
& \widetilde{\chi}_{2}^{0}\left(\widetilde{\chi}_{3}^{0}\right) \rightarrow \widetilde{\chi}_{1}^{0}+\nu+\bar{\nu}(B R \sim 12.36(19.71) \%)
\end{aligned}
$$

where $\ell=e, \mu, \tau ; q=u, d, s, c, b$ and $\nu=\nu_{e}, \nu_{\mu}, \nu_{\tau}$. The two body decay (via loop) includes,

$$
\widetilde{\chi}_{2}^{0}\left(\widetilde{\chi}_{3}^{0}\right) \rightarrow \widetilde{\chi}_{1}^{0}+\gamma\left(B R \sim 3.09\left(1.86 \times 10^{-2}\right) \%\right)
$$

These can give rise to different possible final state signatures at the collider, viz. (2/3/4)lepton $+\mathbb{E}_{T}, 2$-lepton + jets $+\mathbb{E}_{T}, 2$-lepton $+\gamma+\mathbb{E}_{T}, 2 \gamma+\mathbb{E}_{T}$ etc. The lightest chargino i.e. $\widetilde{\chi}_{1}^{ \pm}$also decays via 3 -body decay, viz.

$$
\begin{aligned}
& \tilde{\chi}_{1}^{ \pm} \rightarrow \widetilde{\chi}_{1}^{0}+q+\bar{q}^{\prime}(B R \sim 66.80 \%) \\
& \tilde{\chi}_{1}^{ \pm} \rightarrow \widetilde{\chi}_{1}^{0}+\ell+\nu(B R \sim 33.28 \%)
\end{aligned}
$$

where $q=u, c$ and $q^{\prime}=d, s$. So, we can get multi-lepton $+\mathbb{E}_{T}$, multi-jet $+\mathbb{E}_{T}$ and lepton + jets $+\mathbb{E}_{T}$ final state from chargino pair production. This scenario therefore demonstrates a rich structure of final states which can potentially be probed at the LHC.

Case-3. Instead of decoupled wino-like state as done in case-1, if we rather consider winolike LSP $\left(\widetilde{\chi}_{1}^{0}\right)$, the lightest chargino can have longer lifetime. Heavy Higgs decays to such long lived states give rise to charged tracks at the collider and present another interesting set of collider signatures. To obtain such a long lived chargino, we fix

$$
M_{1}=1000 \mathrm{GeV}, M_{2}=300 \mathrm{GeV}, \mu=500 \mathrm{GeV} \text {. }
$$

It should be noted that the chargino must contain some fraction of higgsino as the heavy Higgs only decays to an admixture of gaugino higgsino states. This necessitates considering low $\mu$ as well as $M_{2}$. We plot the resulting branching ratios in figure 6 . The branching ratio of heavy CP-even Higgs to pair of light charginos is about $6 \%$ while the branching ratio of CP-odd Higgs to chargino pair can be as large as $14 \%$ for this choice of parameters.

Computing the correct chargino lifetime for such analysis however non-trivial. From theoretical calculation [38-40], the wino-like and higgsino-like chargino can have a decay length $\sim$ a few $\mathrm{cm}$ and $\sim \mathrm{mm}-\mu \mathrm{m}$ respectively. In case of the wino-like chargino, the mass difference between the charged and neutral wino state at the tree level is suppressed by a factor of $\sim \frac{m_{W}^{4}}{\Lambda^{3}}$ where $\Lambda \sim \mu, M_{1},\left(\mu, M_{1} \gg m_{W}\right)$. Here the mass splitting arises from loop-corrections to make the chargino long-lived. On the other hand, the mass splitting between the higgsino-like chargino and neutralino arises at the tree level and the one-loop corrections are generally small. Predictions for lifetime from spectrum generator depend on whether such loop corrections are accounted for. Since the loop corrections to the chargino and neutralino mass matrix are absent in Suspect2, the mass difference between wino-like $\widetilde{\chi}_{1}^{ \pm}$and $\widetilde{\chi}_{1}^{0}$ is negligibly small and results in a large the chargino decay length, $\sim \mathrm{km}$ range (green line in left plot of figure 5). In the right plot of figure 5, we compare this mass 

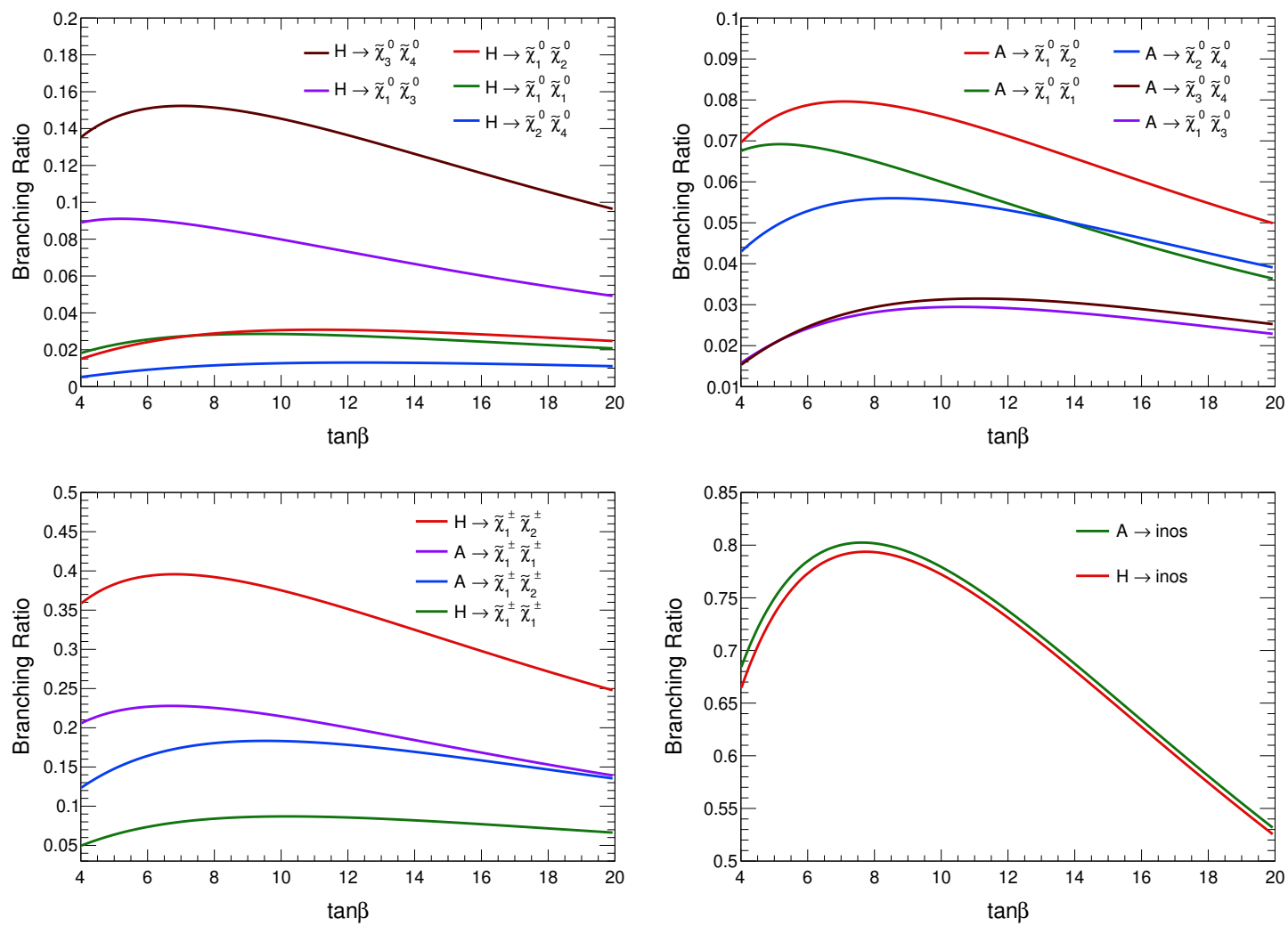

Figure 4. Dominant branching ratios for heavy CP-even and CP-odd Higgs decays to individual electroweakino modes (top panel), the total branching ratio for two heavy Higgs (bottom right), branching ratio of heavy Higgs decaying to charginos (bottom left) for the case-2. The wino, bino, higgsino mass parameters $M_{2}, M_{1}, \mu$ are fixed at 400,300 and $350 \mathrm{GeV}$ and $\tan \beta$ is varied.

splitting between the lightest chargino and neutralino, generated by Suspect2 with the actual one-loop result where the 1-loop data has been taken from the figure 3 of [38] for the case of $\mu=2 M_{2}$. The figure shows that loop correction can make a significant difference to the chargino decay length. However in this work, we have used Suspect2 which does not include these loop corrections.

\section{3 pMSSM random scan}

It has been previously shown that the heavy neutral Higgs boson in the MSSM has a significant branching ratio to susy final states [23, 26, 41, 42]. It is however important to analyse the phenomenologically viable parameter space and understand the signal cross sections of heavy Higgs to susy final states. In order to achieve this, a large scan within 19 dimensional pMSSM using random scan was performed. Table 1 details the ranges of the scan. The points resulting from the random scan were compatible with dark matter direct detection constraints [43], the flavour physics constraints [44, 45], LEP constraints [46] and Higgs signal strengths as well as searches for heavy Higgs at the colliders. These constraints were checked using micromegas [47], HiggsSignals, HiggsBounds [48-52]. It is important 

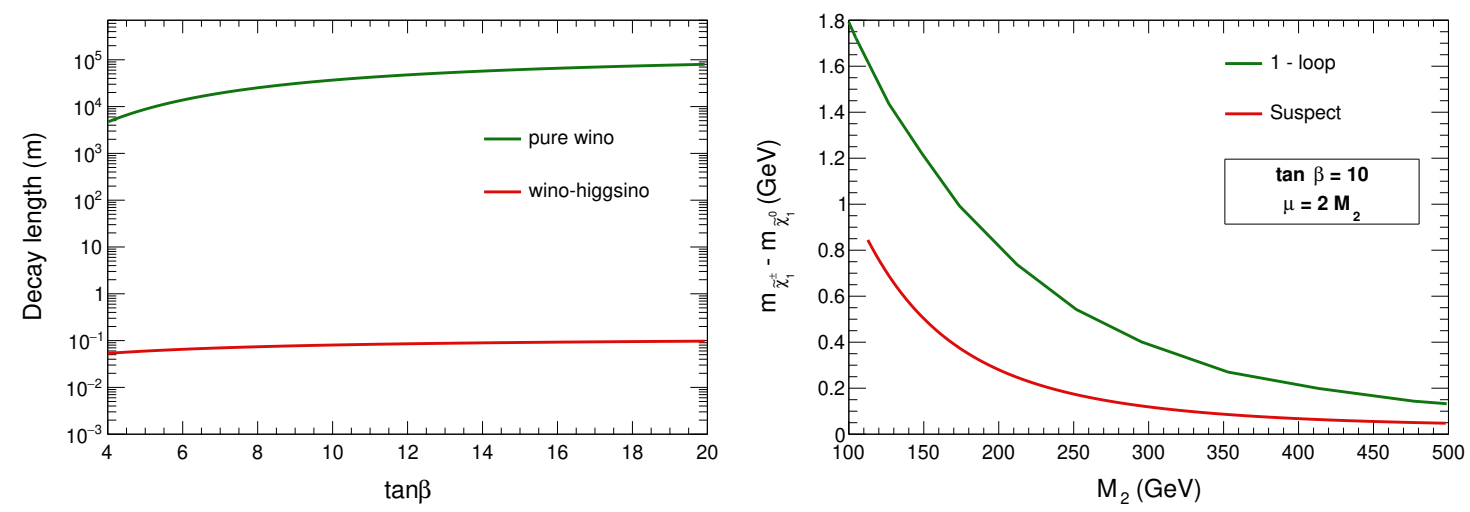

Figure 5. Decay length of a charged wino in pure wino state (green) and mixed state of winohiggsino (red) in Suspect2 (left), and comparison of mass splitting $\left(\Delta m_{\widetilde{\chi}_{1}^{ \pm}-\widetilde{\chi}_{1}^{0}}\right)$ between Suspect2 and 1-loop result [38] (right).

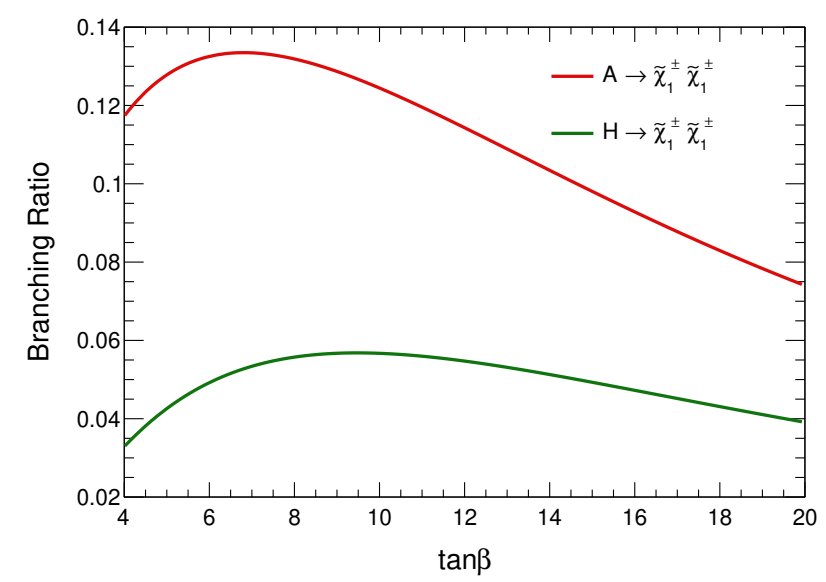

Figure 6. Branching ratio for the decay of heavy Higgs to lightest chargino pair as a function of $\tan \beta$ with fixed, bino, wino and higgsino parameters at $M_{1}=1000 \mathrm{GeV}, M_{2}=300 \mathrm{GeV}, \mu=$ $500 \mathrm{GeV}$.

to note that no constraints on the relic density of the dark matter were applied. In doing so, we allow for the possibility of a non-thermal history in the early Universe. The resulting points were then passed through SUSY-AI [53] and SModelS-1.2.2 [54-58] in order to test against the LHC 8 and $13 \mathrm{TeV}$ constraints. SModels contains up to $36 \mathrm{fb}^{-1}$ results from the susy searches at ATLAS [59-83] and CMS [84-122], therefore, the most recent updates of susy searches with higher luminosity have not been accounted for. For SModels, the production cross sections were computed with Pythia8 [123, 124], the branching ratios with SUSY-HIT [125], NLO corrections to the production cross sections were evaluated using NLL-FAST [126-132]. Finally, it should be noted that there is a basic difference in the way SUSY-AI and SModelS evaluates LHC constraints. SUSY-AI uses machine learning techniques to infer the viability of a MSSM parameter point based on the existing public results from ATLAS pMSSM analysis [133]. SModelS on the other hand uses simplified model technology to decompose the input spectra into the corresponding simplified model 


\begin{tabular}{|c|c||c|c|}
\hline Parameter & range & Parameter & range \\
\hline$M_{1}$ & {$[1,1000]$} & $M_{A}$ & {$[100,2000]$} \\
$M_{2}$ & {$[100,1000]$} & $\mu$ & {$[0,1000]$} \\
$M_{3}$ & {$[700,5000]$} & $\tan \beta$ & {$[1,60]$} \\
$m_{\tilde{e}_{R}, \tilde{\mu}_{R}}$ & 3000 & $m_{\tilde{e}_{L}, \tilde{\mu}_{L}}$ & 3000 \\
$m_{\tilde{\tau}_{R}}$ & {$[80,2000]$} & $m_{\tilde{\tau}_{L}}$ & {$[80,2000]$} \\
$m_{\tilde{q}_{L}}$ & {$[500,10000]$} & $m_{\tilde{q}_{1 L}, \tilde{q}_{2 L}}$ & 3000 \\
$m_{\tilde{t}_{R}}$ & {$[500,10000]$} & $m_{\tilde{b}_{R}}$ & {$[500,10000]$} \\
$A_{b}$ & {$[-2000,2000]$} & $A_{t}$ & {$[-10000,10000]$} \\
$A_{\tau}$ & {$[-2000,2000]$} & $A_{u, d, e}$ & 0 \\
$m_{\tilde{u}_{R}, \tilde{d}_{R}, \tilde{c}_{R}, \tilde{s}_{R}}$ & 3000 & & \\
\hline
\end{tabular}

Table 1. The ranges of MSSM parameters searched by random scan. The mass scales are in $\mathrm{GeV}$ units.

\begin{tabular}{|c|c|}
\hline Constraint name & Range \\
\hline$B \rightarrow X_{s} \gamma$ & {$[2.583,4.057] \times 10^{-4}$} \\
$B_{s} \rightarrow \mu \mu$ & {$[1.2912,4.8974] \times 10^{-9}$} \\
\hline
\end{tabular}

Table 2. Flavour physics constraints used in our random scan. We consider $10 \%$ uncertainty around central value as theoretical error which is added in quadrature with the experimental error to get the total error. This range is obtained by including two times this total error $(2 \sigma)$.

topologies. It compares the theory cross sections resulting from decomposition procedure with the corresponding experimental results. While SUSY-AI is more robust than SModelS in this aspect, SModelS contains a more comprehensive and updated database of results compared to SUSY-AI. Drawing outright comparison between the two codes is therefore non-trivial.

For direct detection, we utilised the latest results form the XENON1T collaboration [43], while the theory parameter space was appropriately rescaled by $\zeta$ where $\zeta$ is defined by $\zeta=\Omega h_{\text {central }}^{2} / \Omega h_{\text {theory }}^{2}$, where $\Omega h_{\text {central }}^{2}=0.1189$ [134]. Here, we use a parametric form of the direct detection cross section similar to [23]. We would like to mention here that we also do mcmc scan in the MSSM parameter space which give similar results.

\subsection{Scan results}

The results of our scan in particular show that $\tan \beta \gtrsim 20$ is ruled out for a heavy Higgs mass $m_{A}<1 \mathrm{TeV}$. On the other hand, all values of $5<\tan \beta<60$ are allowed for $m_{A} \gtrsim$ $1.65 \mathrm{TeV}$. We will not further discuss the features of the parameter space, however this exercise demonstrates that particularly in the electroweak sector a large parameter space remains unconstrained by the current experimental searches (see appendix A for details).

With this allowed parameter space as a base for our further studies, we concentrate on mono-X signatures at HL-LHC. As has been demonstrated before [26, 41], the heavy Higgs decays to susy particles mostly lead to mono-X final states after accounting for 
phenomenological constraints, hence we expect these signatures to be the most promising ones in the search for heavy Higgs to susy decays. More concretely, we calculate the yield at HL-LHC configuration for the following processes,

$$
\begin{array}{rlrl}
g g & \rightarrow H / A & \rightarrow \widetilde{\chi}_{1}^{0} \widetilde{\chi}_{2,3}^{0}, & \tilde{\chi}_{2,3}^{0} \rightarrow \tilde{\chi}_{1}^{0}(h / Z), \\
b \bar{b} \rightarrow H / A \rightarrow \widetilde{\chi}_{1}^{0} \tilde{\chi}_{2,3}^{0}, & \tilde{\chi}_{2,3}^{0} \rightarrow \widetilde{\chi}_{1}^{0}(h / Z) .
\end{array}
$$

In figure 7 , we show event yield at $3 \mathrm{ab}^{-1}$ for the mono- $\mathrm{Z}$ and mono-h final states arising from aforementioned channels as a function of $m_{A}$ and $\tan \beta$. We compute the heavy Higgs production cross section using SusHi [135]. Furthermore, we divide event yields in two categories corresponding to ggH and bbH Higgs production processes. The production cross section is in general higher for the $g g$ initiated Higgs prodction in the low $m_{A}$ and low $\tan \beta$ region, therefore leads to higher event yield. Since heavy Higgs coupling to the down type quarks is proportional to $\tan \beta$, the event yield in case of $b \bar{b}$ initiated production increases for larger $\tan \beta$ values. In general the event yield reaches up to $10^{5}$ events in ggH mode for Higgs masses less than $1 \mathrm{TeV}$, for bbH mode even larger masses can yield substantial $\left(\sim 10^{4}\right)$ number of events. This motivates development of separate dedicated search strategies for the $\mathrm{ggH}$ and $\mathrm{bbH}$ modes, where for the bbH mode the presence of additional b-jets could be exploited to gain sensitivity as compared to the ggH mode.

The above discussed processes correspond to prompt decays of neutralinos. In correspondence to the case- 3 in section 2 , we also compute the production cross section for heavy Higgs decays to long-lived $\widetilde{\chi}_{1}^{ \pm}$at the HL-LHC. For this, we consider the process,

$$
g g / b \bar{b} \rightarrow(H / A) \rightarrow \widetilde{\chi}_{1}^{ \pm} \widetilde{\chi}_{2}^{\mp}, \widetilde{\chi}_{2}^{\mp} \rightarrow W^{\mp} \widetilde{\chi}_{1}^{0} .
$$

We show the chargino production cross section times $\operatorname{Br}\left(\widetilde{\chi}_{2}^{\mp} \rightarrow W^{\mp} \widetilde{\chi}_{1}^{0}\right)$ in the plane of $m_{\widetilde{\chi}_{2}^{ \pm}}$vs $\left(m_{\widetilde{\chi}_{1}^{ \pm}}-m_{\widetilde{\chi}_{1}^{0}}\right)$ (top left), $m_{\widetilde{\chi}_{1}^{ \pm}}$vs decay length (top right) and $m_{H}$ vs $\tan \beta$ (bottom panel) in figure 8. Of particular importance here is the impact of two loop corrections on the chargino-neutralino mass splitting and associated change in the chargino decay length. We have used Suspect2 to compute the MSSM mass spectrum, this version does not include loop corrections to chargino masses. For small chargino-neutralino mass difference these corrections are particularly important. Therefore, while the qualitative features of this final state are robust, the quantitative estimates in particular for the chargino lifetimes are subject to change and have not been accounted for within this work. ${ }^{1}$ figure 8 demonstrates the chargino-neutralino mass difference (top left), the chargino decay length (top right), and associated cross section where the heavier chargino decays to a $\mathrm{W}$ boson (bottom panel). It can be seen that in general the cross sections for these processes are large and given the rather low background for searches involving disappearing track and heavy stable charged particles, such final states present an interesting avenue for heavy Higgs searches.

Apart from decays of heavy Higgs to susy final states, it is also possible to produce heavy Higgs from decays of susy particles. We alluded to this possibility in section 2 while

\footnotetext{
${ }^{1}$ We have also checked our scan results using the most recent version of Suspect3-beta [136] which accounts for full one-loop and dominant two-loop radiative corrections to the masses of electroweakinos. We get similar results as with Suspect2. However, the version Suspect3-beta is under development. Therefore, we do not use this in our analysis.
} 

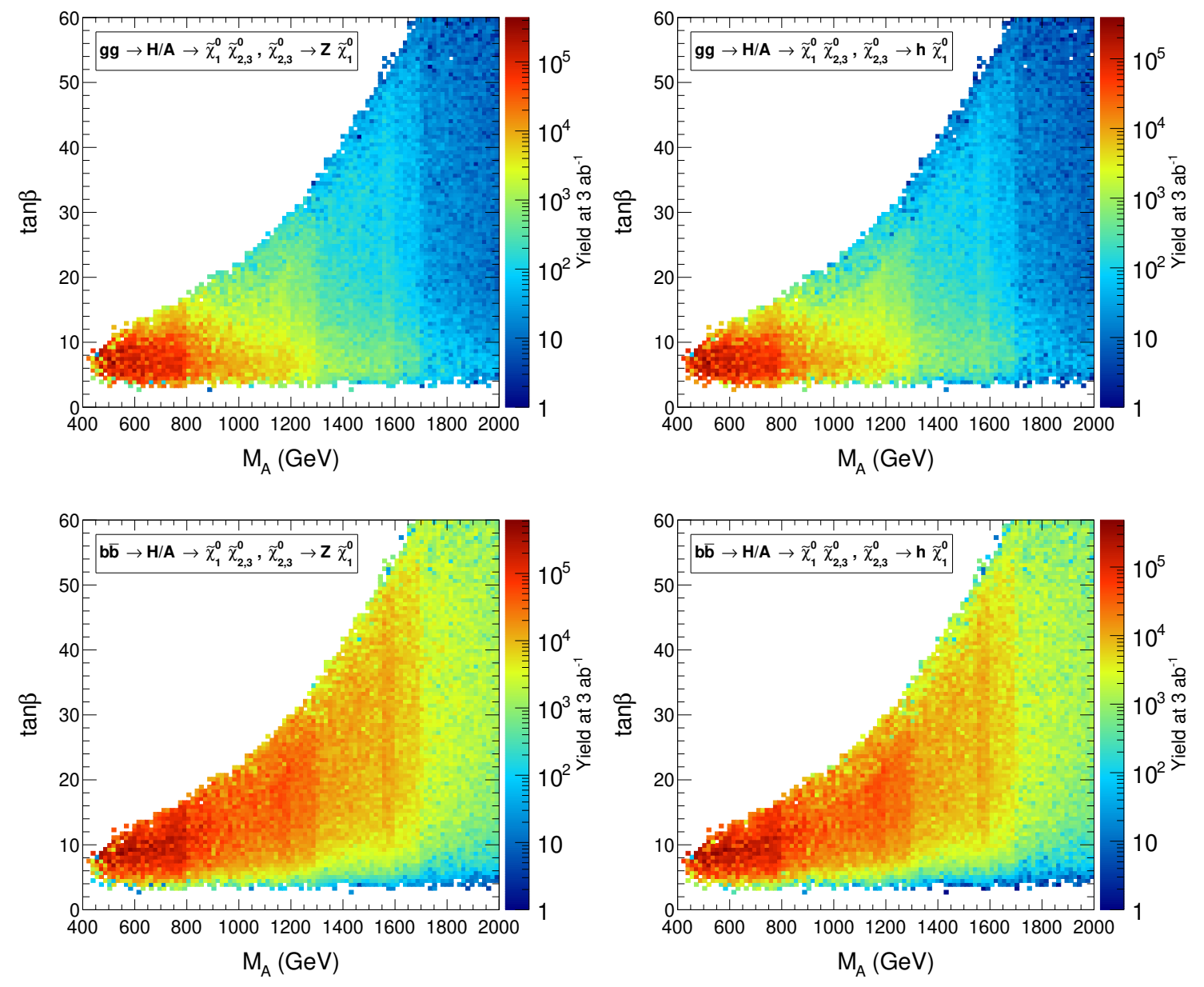

Figure 7. Event yield at $3 \mathrm{ab}^{-1}$ of integrated luminosity for all points passing phenomenological constraints for gluon fusion process leading to mono-Z/h final state (top left, top right panel) while the $\mathrm{bbH}$ production mode leading to mono- $\mathrm{Z} / \mathrm{h}$ final state (bottom left, right panel). The plots demonstrate that bbH production in general leads to high event yields for moderate $\tan \beta$ due to enhanced Yukawa couplings.

discussing several benchmark scenarios for heavy Higgs to electroweakino final states. It is also possible that heavy Higgs emerges from cascade decays of susy particles apart from electroweakinos. We explicitly demonstrate the branching ratios for $\widetilde{t_{2}} \rightarrow \widetilde{t_{1}} H$, $\widetilde{t_{2}} \rightarrow \widetilde{b_{1}} H^{+}, \widetilde{b_{2}} \rightarrow \widetilde{b_{1}} H$ and $\widetilde{b_{2}} \rightarrow \widetilde{t_{1}} H^{-}$. We calculate these branching ratios with Susyhit for the parameter space regions which satisfy all of the previously discussed experimental constraints. In figure 9 , we show the branching ratios for above mentioned decay modes. ${ }^{2}$ For convenience we highlight points where heavy stop/sbottom masses are less than $2 \mathrm{TeV}$, while plotting all points which have a non-zero branching ratio to heavy Higgs (grey). The decay rate can reach up to $8-10 \%$, however this generally requires a stop/sbottom heavier than $2 \mathrm{TeV}$, thus limiting the reach of LHC for such processes. A detailed investigation of

\footnotetext{
${ }^{2}$ For this plot we do not sum up CP-even and CP-odd Higgs in final state, in case that is done, the branching ratios will be almost twice as large for these processes.
} 

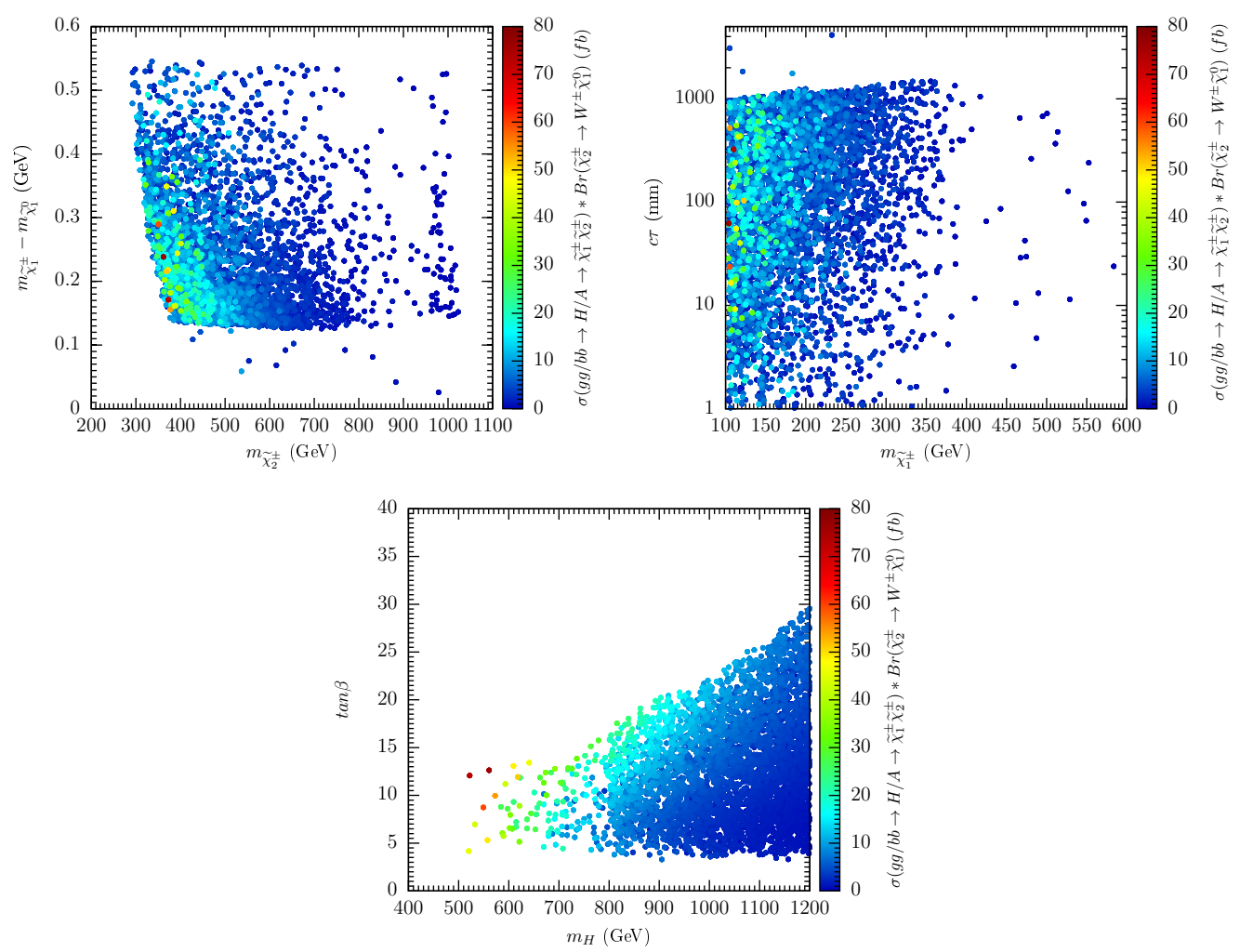

Figure 8. The event yield for $\widetilde{\chi}_{1}^{ \pm}$production from heavy Higgs in $m_{\widetilde{\chi}_{2}^{ \pm}}-\left(m_{\widetilde{\chi}_{1}^{ \pm}}-m_{\widetilde{\chi}_{1}^{0}}\right)$, decay length- $m_{\widetilde{\chi}_{1}^{ \pm}}$and $m_{H}-\tan \beta$ plane for all points passing phenomenological constraints. The chargino-neutralino mass difference does not include loop corrections discussed in the text. Event yields as a function of heavier chargino and chargino-neutralino mass difference (top left), as a function of lightest chargino mass and lifetime (top right) and as a function of heavy Higgs mass and $\tan \beta$ are displayed.

this analysis is beyond the scope of this study however we stress the need of characterising this parameter space further and understand potential for heavy Higgs final states at the LHC. These present also an additional opportunity for exploring heavy Higgs and susy sectors at the $100 \mathrm{TeV}$ collider.

\subsection{Mono-Z event kinematics}

Having motivated an in-depth analysis of mono-X final states in the previous subsection, we now turn our attention to understanding the salient kinematical features for these final states emerging via the decay of heavy Higgs. We will rely on these kinematic features for analysis optimization in the following sections. We expect the kinematics to be different for electroweakino resulting from decays of heavy Higgs against the ones produced via SM mediators. The reason being the heavy Higgs is produced on-shell and hence should leave an imprint of resonant production on the final state, the SM mediators responsible for direct electroweakino production on the other hand are always off-shell. In order to understand potential differences between the Standard Model (SM) mediated and the Higgs mediated 

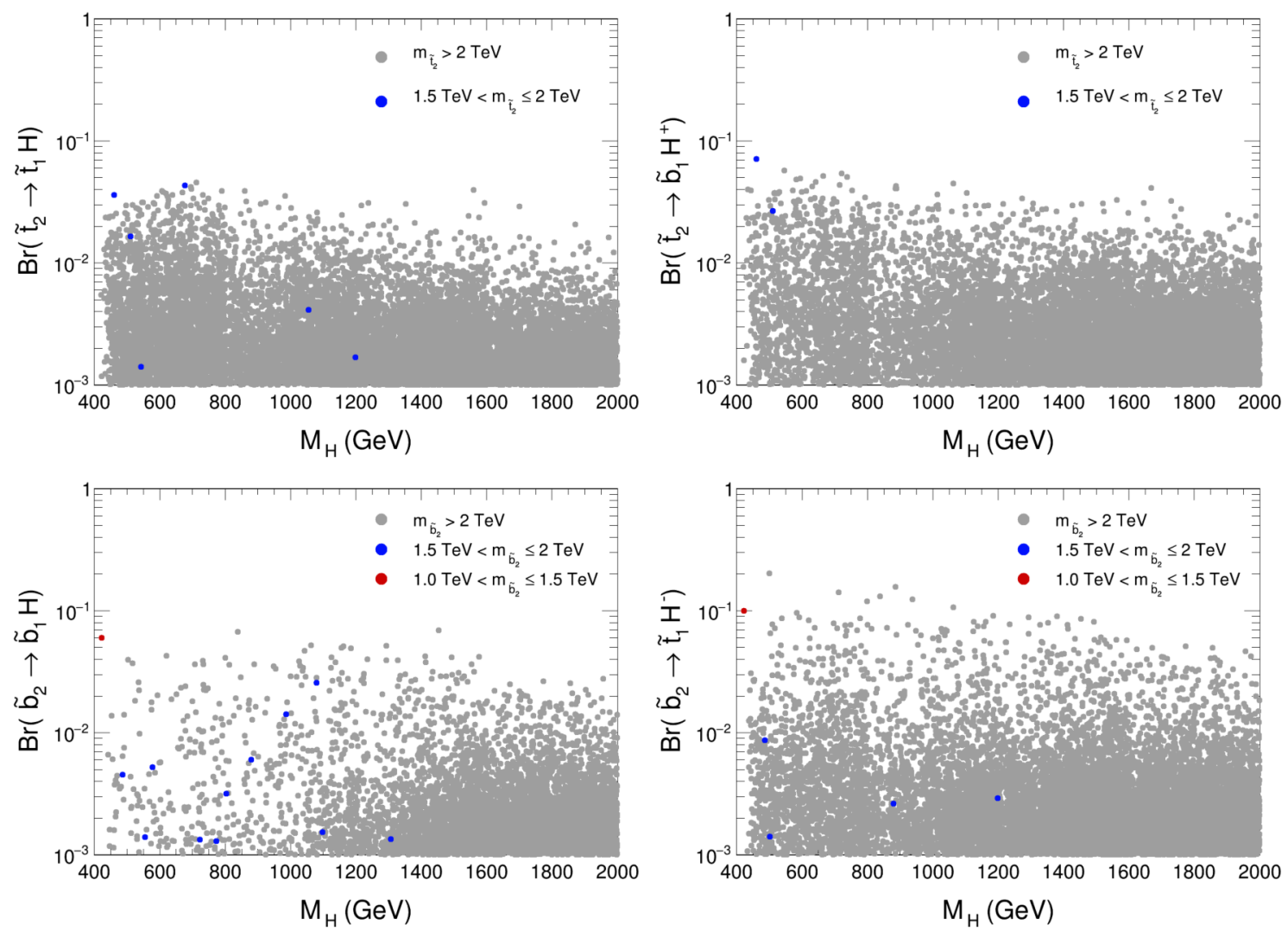

Figure 9. Branching ratios of heavy Higgs production from heavy squarks for all points passing phenomenological constraints. Points in grey are all points for which we find non-zero branching ratio to heavy Higgs final state, we highlight in blue points for which heavy stop/sbottom mass is less than $2 \mathrm{TeV}$.
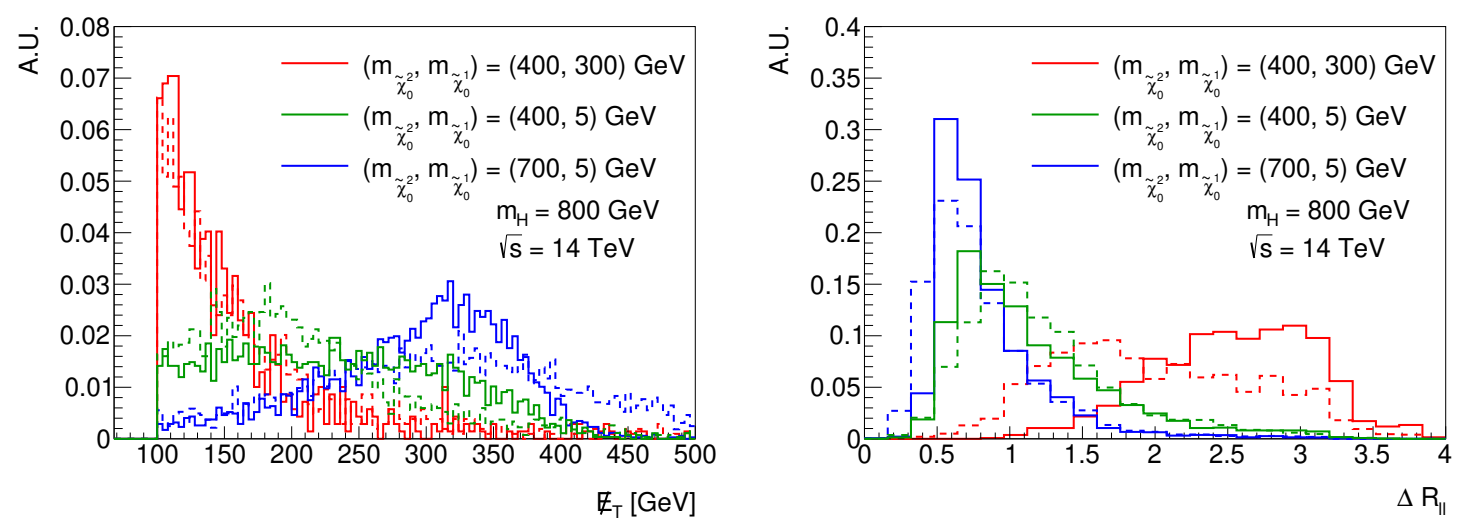

Figure 10. Normalised distributions for missing energy of the events (left) and $\Delta R$ between two leptons (right) with preselections as defined in the text for three different benchmark points. The dashed lines represent production via SM mediated processes and the solid lines represent production via heavy Higgs channel. 

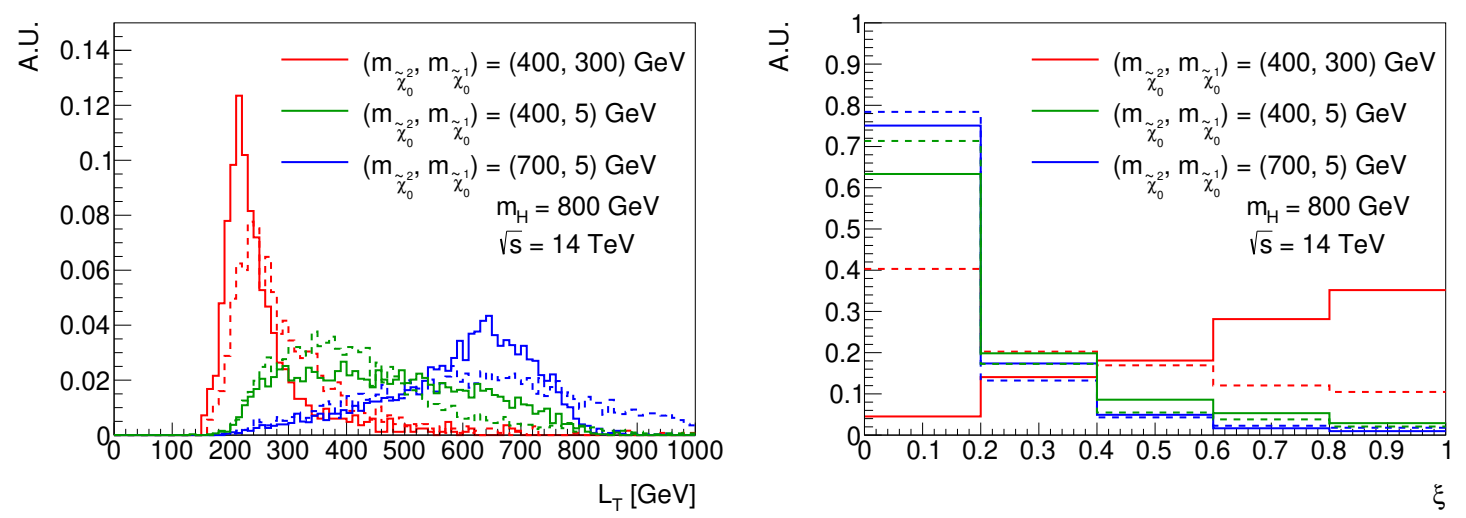

Figure 11. Normalised distributions for $L_{T}$ of the events with preselections as defined in the text (left) and $\xi$ (right) for three different benchmark points. The dashed lines represent production via SM mediated processes and the solid lines represent production via heavy Higgs channel.

production of the electorweakinos, in this section, we present several kinematic features for a few benchmark scenarios. We choose a fixed heavy Higgs mass at $800 \mathrm{GeV}$, and vary the masses of $\widetilde{\chi}_{2}^{0}$ and $\widetilde{\chi}_{1}^{0}$. We simulate the Higgs boson production, subsequent decays and hadronise the events using Pythia8. We consider only gluon fusion channel. The heavy Higgs is decayed to $\widetilde{\chi}_{2}^{0}+\widetilde{\chi}_{1}^{0}$ using $100 \%$ branching ratio. We further simulate decays of $\widetilde{\chi}_{2}^{0}$ to $\mathrm{Z}+\widetilde{\chi}_{1}^{0}$ also using $100 \%$ branching ratios, furthermore, $\mathrm{Z}$ is decayed only to electron and muon final states. The events are passed through Delphes [137] using default ATLAS card and the resulting final state in dilepton $+\mathbb{E}_{T}$ is analysed. For all the distributions below, we choose events with exactly two leptons, less than equal to 2 jets in the final state with $p_{T}\left(j_{1}\right)>30 \mathrm{GeV}$, and $\mathbb{E}_{T}>100 \mathrm{GeV}$.

In figure 10, we plot the missing energy (left) and the $\Delta R_{\ell \ell}{ }^{3}$ between two leptons (right) at reconstructed level. The dashed lines represent direct production of $\widetilde{\chi}_{2}^{0}, \widetilde{\chi}_{1}^{0}$ while the solid lines represent the production of same particles except via the heavy Higgs boson whose mass is fixed at $800 \mathrm{GeV}$. Three different benchmark points are chosen such that the heavy Higgs always decays on shell to $\widetilde{\chi}_{2}^{0}, \widetilde{\chi}_{1}^{0}$. In addition care has been taken to choose different masses to represent a range of different boosts received by final state leptons. The combination $\left(m_{\widetilde{\chi}_{2}^{0}}, m_{\widetilde{\chi}_{1}^{0}}\right)=(400,300) \mathrm{GeV}$ corresponds to the maximal allowed $\left(\widetilde{\chi}_{2}^{0}, \widetilde{\chi}_{1}^{0}\right)$ masses such that the decays of Higgs boson to electroweakinos and electroweakino to $\mathrm{Z}$ are on shell. ${ }^{4}$ The missing energy distribution for direct electroweakino production or production via heavy Higgs is very similar. This is because the $\mathrm{Z}$ in the final state is almost produced at rest, this is reflected in the $\Delta R_{\ell \ell}$ distribution, which peaks for maximum values. When the mass difference between $\widetilde{\chi}_{2}^{0}, \widetilde{\chi}_{1}^{0}$ is increased drastically, for $\left(\widetilde{\chi}_{2}^{0}, \widetilde{\chi}_{1}^{0}\right)=$ $(400,5)$, we find a very different situation. Here, the missing energy is generally harder,

\footnotetext{
${ }^{3}$ The distance between two particles $a$ and $b$ in the $\eta-\phi$ plane, $\Delta R_{a b}$ is defined as $\Delta R_{a b}=\sqrt{\Delta \eta_{a b}^{2}+\Delta \phi_{a b}^{2}}$ where $\Delta \eta_{a b}$ is the distance in the pseudorapidity plane and $\Delta \phi_{a b}$ is the azimuthal angle separation between the particles $a$ and $b$.

${ }^{4}$ It is possible that the electroweakinos can decay via off shell $\mathrm{Z}$ boson, however we do not consider this possibility and associated kinematics in this work.
} 
the corresponding $\Delta R_{\ell \ell}$ distribution shows a more collimated pair of leptons compared to the susy counterpart. Finally, we change the situation completely and consider the largest $\widetilde{\chi}_{2}^{0}$ mass allowed for on shell Higgs, which leads to combination $\left(\widetilde{\chi}_{2}^{0}, \widetilde{\chi}_{1}^{0}\right)=(700,5)$. In this case, the MET generated by the SM mediated process is somewhat harder than the corresponding Higgs mediated process. We thus see an interesting complementarity between kinematic distributions generated by SM mediated and Higgs mediated processes. Also, we present a discussion of the difference between parton level and detector level kinematic feature of $\mathbb{E}_{T}$ in appendix B.

Finally, to conclude this discussion, we demonstrate in figure 11 two more kinematic variables which are derived using the basic measurable quantities in the dilepton plus missing energy final state events. The two quantities are $L_{T}$ which is the scalar sum of lepton $p_{T}$ and missing energy of the event and the $\xi$ defined by $\xi=\left|p_{T, \ell \ell}-\mathbb{E}_{T}\right| / p_{T, \ell \ell}[26,32]$ where $p_{T, \ell \ell}$ is the transverse momentum of the dilepton system. While $L_{T}$ should give us an indication of the presence of any resonance, the variable $\xi$ is an indicator of the momentum imbalance in the system. For the $L_{T}$ distribution, $\left(\widetilde{\chi}_{2}^{0}, \widetilde{\chi}_{1}^{0}\right)=(400,300)$ the $L_{T}$ is soft, it peaks around $200-300 \mathrm{GeV}$, with a long tail, the distributions for the Higgs and SM mediated processes are similar. For the other two points which correspond to large mass difference between $\widetilde{\chi}_{2}^{0}$ and $\widetilde{\chi}_{1}^{0}$, the $L_{T}$ distribution exhibits a clear end point for Higgs mediated process over the SM mediated process. Finally, the $\xi$ distribution shows an interesting dependence on the mass difference, for the benchmarks with high mass difference, the distribution peaks for low values, while for small mass difference it peaks for high values. For $\left(m_{\widetilde{\chi}_{2}^{0}}, m_{\widetilde{\chi}_{1}^{0}}\right)=(400,300) \mathrm{GeV}$, the leptons are produced with low $p_{T}$ and therefore the final state $\mathbb{E}_{T}$ is generated from the jets. Correspondingly, the $\xi$ distribution peaks near 1 as opposed to other two benchmark points.

\subsection{LLCP kinematics}

As discussed in section 3.1, heavy Higgs decays to long lived charged particles (LLCP) can result in a substantial signal at the HL-LHC. In order to illustrate the salient kinematical differences between LLCP produced via decays of the heavy Higgs and via Drell-Yan process at the LHC, in this section we take a few benchmark points and analyse their kinematics. We use a similar setup as for the mono- $\mathrm{Z}$ final state in constructing the simplified model. Concretely, we generate the process $p p \rightarrow H \rightarrow \widetilde{\chi}_{1}^{ \pm} \widetilde{\chi}_{2}^{ \pm}, \widetilde{\chi}_{2}^{ \pm} \rightarrow W^{ \pm} \widetilde{\chi}_{1}^{0}, \widetilde{\chi}_{1}^{ \pm} \rightarrow \widetilde{\chi}_{1}^{0} W^{*}$. The W boson has been decayed inclusively. For the Drell-Yan production of charginos, we simulate pair production of lightest chargino. ${ }^{5}$ We generate signal and hadronize with Pythia8, we perform no detector simulation and present the kinematics at generator level. The heavy Higgs mass has been fixed to $1.8 \mathrm{TeV}$, and the mass of heavy chargino $\left(\widetilde{\chi}_{2}^{ \pm}\right)$is fixed to $434 \mathrm{GeV}$. We then vary the mass of lighter chargino $\left(\widetilde{\chi}_{1}^{ \pm}\right)(122,222,322 \mathrm{GeV})$ and fix the chargino lifetime to $3 \mathrm{~mm}$. For a chargino to be long lived, the mass difference between chargino and LSP must be small, we therefore fix the mass difference between $\widetilde{\chi}_{1}^{ \pm}, \widetilde{\chi}_{1}^{0}$ to be $0.5 \mathrm{GeV}$.

\footnotetext{
${ }^{5}$ We neglect other production channels as $p p \rightarrow \widetilde{\chi}_{1}^{ \pm} \widetilde{\chi}_{1}^{ \pm}$has the highest production cross section.
} 

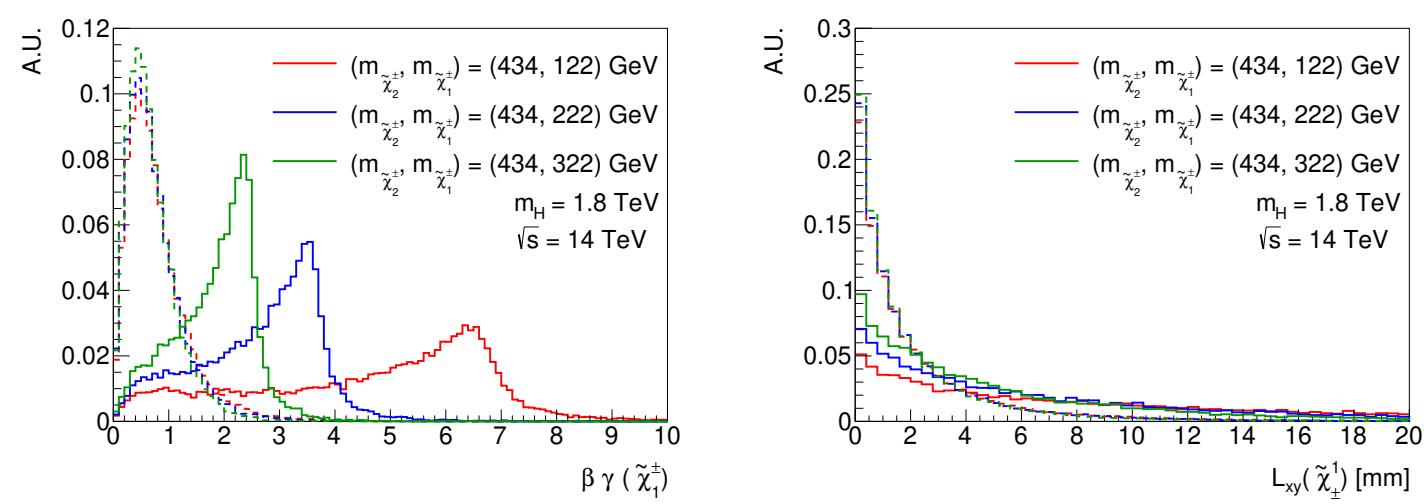

Figure 12. The boost received by lightest chargino (left) and the resulting transverse displacement distribution (right) when chargino is produced in Drell-Yan processes (dashed lines) vs. chargino produced via decays of heavy Higgs (solid lines). The mass of heavy Higgs and heavier chargino is fixed at $1.8 \mathrm{TeV}$ and $434 \mathrm{GeV}$ respectively, the mass of lighter chargino is varied, mass difference between $\widetilde{\chi}_{1}^{ \pm}, \widetilde{\chi}_{1}^{0}$ is fixed at $0.5 \mathrm{GeV}$.

Figure 12 (left) shows resulting chargino boost distributions without any cuts. It is clear that the boost received by the lightest chargino depends on the mass hierarchy between heavy Higgs, and chargino. ${ }^{6}$ The lightest chargino with a mass of $\sim 100 \mathrm{GeV}$ produced via decay of heavy Higgs (solid red line) is maximally boosted given the large mass difference between heavy Higgs and the chargino. This boost gets smaller and smaller as the mass of the chargino increases to $\sim 200 \mathrm{GeV}$ (solid blue) and $\sim 300 \mathrm{GeV}$ (solid green). For the chargino pair production via the Drell-Yan process (dashed lines), the boost is much smaller compared to the heavy Higgs decays case and the three masses show no significant differences. Such varied boost distribution results in different transverse decay lengths of chargino as depicted in figure 12 (right). It demonstrates that the chargino arising from heavy Higgs decays traverse longer distances through the detector.

\section{Collider analysis}

As shown in the previous section, the mono-X signatures arising from heavy Higgs decays can be of an interest at the LHC. In this section, we will mainly focus on $p p \rightarrow H / A \rightarrow \widetilde{\chi}_{1}^{0}+$ $\left(\widetilde{\chi}_{2,3}^{0}\right),\left(\widetilde{\chi}_{2,3}^{0}\right) \rightarrow \widetilde{\chi}_{1}^{0}+(Z / h)$ which leads to mono- $Z$ and mono- $h$ final state. Furthermore, the $\mathrm{Z}$ and $\mathrm{h}$ bosons can decay to several different SM final states. Among them, we choose 3 possible decay modes for our analysis, mainly in terms of cleanliness and/or larger branching ratio viz. (a) $Z \rightarrow \ell \ell$, (b) $h \rightarrow b \bar{b}$ and (c) $h \rightarrow \gamma \gamma$ (figure 137), which leads to $\ell \ell+\mathbb{E}_{T}, b \bar{b}+\mathbb{E}_{T}$ and $\gamma \gamma+\mathbb{E}_{T}$ final states respectively. In order to uncover such a signal at colliders, it needs to be discriminated against not just the SM backgrounds but electroweakino production via SM mediators which leads to the same finals states, potentially with different kinematics. To this end, we consider two kinds of background processes viz. (i) the usual SM background production e.g. $p p \rightarrow V V, t \bar{t}, V h, t \bar{t} h, t \bar{t} V$

\footnotetext{
${ }^{6}$ In principle it also depends on the mass of the heavy chargino, however it has been fixed for kinematic studies.

${ }^{7}$ JaxoDraw [138] has been used to generate all the Feynman diagrams in this paper.
} 


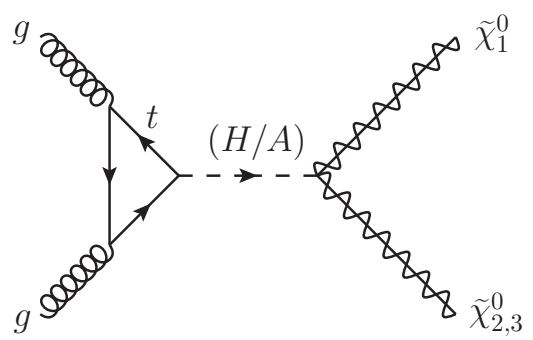

(a)

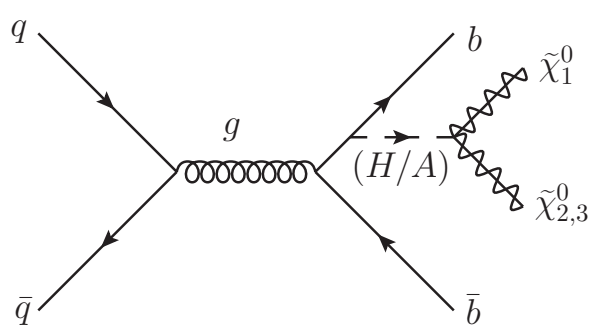

$(c)$

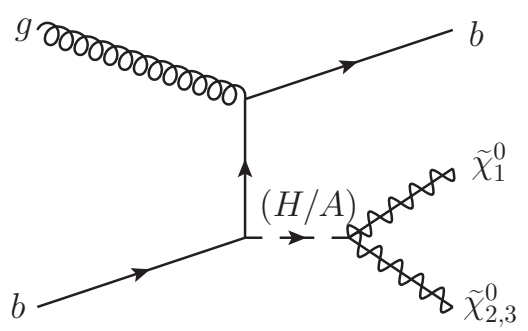

$(e)$

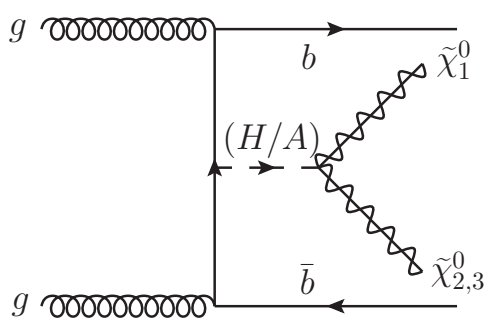

(b)

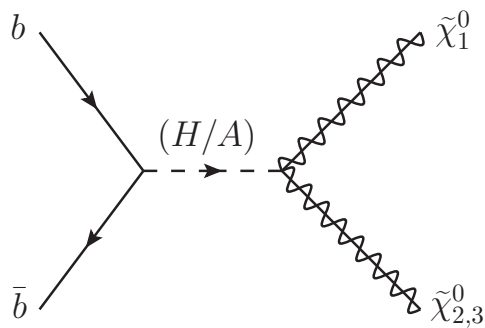

$(d)$

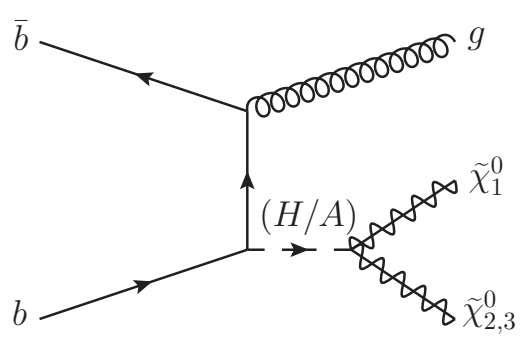

$(f)$

Figure 13. The Feynman diagram of signal processes from (a) $g g$ fusion process; $4 \mathrm{~F}$ (b) $g g \rightarrow b \bar{b} H$ and (c) $q \bar{q} \rightarrow b \bar{b} H$ production; $5 \mathrm{~F}$ (d) $b \bar{b} \rightarrow H$ (LO), (e) $g b \rightarrow b H$ and (f) $b \bar{b} \rightarrow g H$ production. The $\widetilde{\chi}_{2,3}^{0}$ then decays to $\widetilde{\chi}_{2,3}^{0} \rightarrow \widetilde{\chi}_{1}^{0}+(Z / h)$.

etc. where $V$ denotes $W^{ \pm}$and $Z$ boson, and, (ii) susy production via SM mediators, which leads to the same final state as the signal processes. The second kind of background i.e. susy production, mainly comes from the direct production of the electroweakino pairs via the $Z$ and $W$ bosons in the s-channel or via squarks in the t-channel e.g. $p p \rightarrow \widetilde{\chi}_{i}^{0} \widetilde{\chi}_{j}^{0}, \widetilde{\chi}_{i}^{0} \widetilde{\chi}_{k}^{ \pm}$ and $\tilde{\chi}_{k}^{ \pm} \widetilde{\chi}_{k}^{\mp}$, where $i, j=1,2,3,4$ and $k=1,2$ as depicted in figure 14 . We would like to mention here that these susy backgrounds have so far been not accounted for in the existing mono- $Z$ and mono- $h$ phenomenology arising from decays of heavy Higgs [26, 27].

We generate SM backgrounds with generator level cuts as specified in the appendix C, while the susy backgrounds are generated without any generator level cuts.

In order to demonstrate possible optimisation of analysis, we choose the following two benchmark points $(\mathrm{BPs})^{8}$ in the allowed MSSM parameter space, given in table 3 where we

\footnotetext{
${ }^{8}$ Since the LSP is lighter here, we check against CRESST-II [139] limits before choosing these benchmark points.
} 


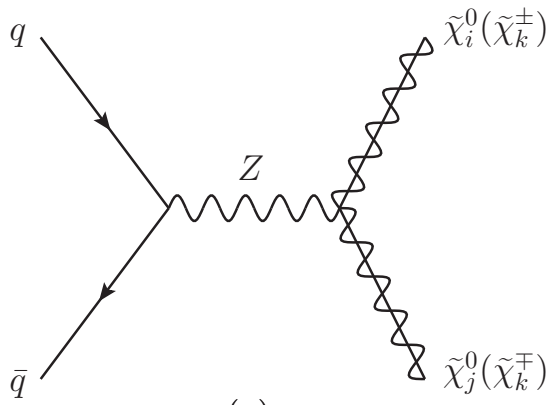

$(a)$

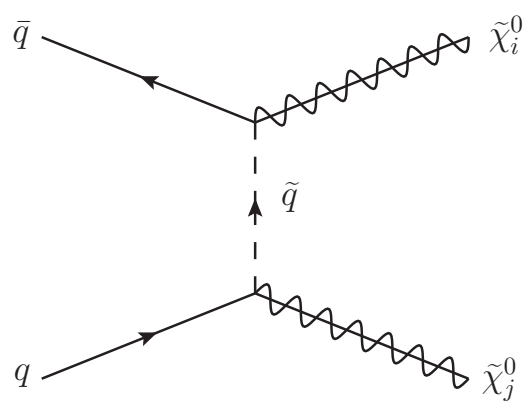

$(c)$

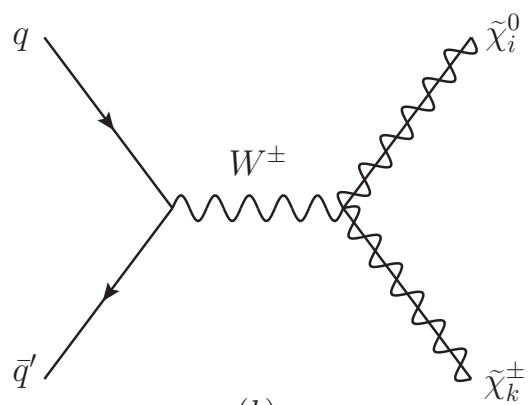

(b)

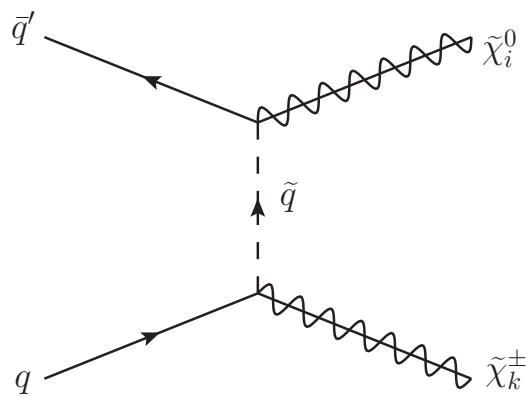

$(d)$

Figure 14. The Feynman diagram of susy background production e.g $p p \rightarrow \widetilde{\chi}_{i}^{0} \widetilde{\chi}_{j}^{0}\left(\widetilde{\chi}_{k}^{ \pm} \widetilde{\chi}_{k}^{\mp}\right)$ and $p p \rightarrow \widetilde{\chi}_{i}^{0} \widetilde{\chi}_{k}^{ \pm}$with $i, j=1,2,3,4$ and $k=1,2$, in the s-channel ((a) and (b) respectively) and in the t-channel ((c) and (d) respectively). Here, the s-channel is the dominant production mode because the squarks in the t-channel are heavier for our chosen benchmark points.

also mention the branching ratios and cross sections relevant for our mono- $Z$ and mono- $h$ analysis. The common soft parameters for both of the BPs are:

$$
\begin{aligned}
& M_{1}=5 \mathrm{GeV}, M_{2}=1.1 \mathrm{TeV}, \mu=243.2 \mathrm{GeV}, M_{3}=2 \mathrm{TeV}, \\
& M_{\tilde{Q}_{1_{L}}, \tilde{Q}_{2_{L}}}=M_{\tilde{u}_{R}, \tilde{d}_{R}, \tilde{c}_{R}, \tilde{s}_{R}}=M_{\tilde{e}_{L}, \tilde{\mu}_{L}, \tilde{e}_{R}, \tilde{\mu}_{R}}=3 \mathrm{TeV}, M_{\tilde{Q}_{3_{L}}}=4.9 \mathrm{TeV}, \\
& A_{t}=-3.7 \mathrm{TeV}, A_{b}=-1.1 \mathrm{TeV}, A_{\tau}=-1.5 \mathrm{TeV}, A_{e, \mu, u, d, c, s}=0, \\
& M_{\tilde{\tau}_{L}}=961.5 \mathrm{GeV}, M_{\tilde{\tau}_{R}}=1.1 \mathrm{TeV}, M_{\tilde{t}_{R}}=5.9 \mathrm{TeV}, M_{\tilde{b}_{R}}=2 \mathrm{TeV}
\end{aligned}
$$

These BPs are allowed by all the experimental constraints except the relic density constraint. The BPs are chosen such that the heavy Higgs bosons have a significant branching ratio into susy final states. Given the hierarchy of the bino, wino and higgsino mass parameters $\left(M_{1}<\mu<M_{2}\right)$, the LSP is primarily bino-like whereas the $\widetilde{\chi}_{2}^{0}$ and $\widetilde{\chi}_{3}^{0}$ contains mostly the neutral higgsino components. Also, the $\widetilde{\chi}_{1}^{ \pm}$contain the charged higgsino fields, while the heavy electroweakinos i.e. $\widetilde{\chi}_{4}^{0}$ and $\widetilde{\chi}_{2}^{ \pm}$, becomes wino-like due to large value of $M_{2}$ parameter.

In the following subsections, we perform an optimised analysis for our chosen benchmark points for mono- $Z$ and mono- $h$ search channels. Besides, we divide our analysis in two parts, b-veto category where there is no extra b-tagged jet along with the final state particles and b-tag category where we demand the presence of an additional b-jet. We 


\begin{tabular}{|c|c|c|c|c|c|}
\hline $\begin{array}{c}\text { Benchmark } \\
\text { Points }\end{array}$ & Parameters & Mass $(\mathrm{GeV})$ & $\begin{array}{l}\text { Branching } \\
\text { Ratio (\%) }\end{array}$ & Processes & Cross-section (fb) \\
\hline BP1 & $\begin{array}{c}M_{A}=650 \mathrm{GeV} \\
\tan \beta=10.8 \\
\text { eq. }(4.1)\end{array}$ & $\begin{array}{c}m_{\widetilde{\chi}_{1}^{0}}=3.23 \\
m_{\widetilde{\chi}_{2}^{0}}=251.17 \\
m_{\widetilde{\chi}_{3}^{0}}=255.55 \\
m_{\widetilde{\chi}_{1}^{ \pm}}=248.20 \\
m_{H}=650.31\end{array}$ & $\begin{array}{c}\operatorname{BR}\left(H(A) \rightarrow \widetilde{\chi}_{1}^{0} \widetilde{\chi}_{2}^{0}\right)=11.00(16.11) \\
\operatorname{BR}\left(H(A) \rightarrow \widetilde{\chi}_{1}^{0} \widetilde{\chi}_{3}^{0}\right)=15.25(9.46) \\
\operatorname{BR}\left(\widetilde{\chi}_{2}^{0} \rightarrow \widetilde{\chi}_{1}^{0} Z\right)=50.74 \\
\operatorname{BR}\left(\widetilde{\chi}_{2}^{0} \rightarrow \widetilde{\chi}_{1}^{0} h\right)=49.26 \\
\operatorname{BR}\left(\widetilde{\chi}_{3}^{0} \rightarrow \widetilde{\chi}_{1}^{0} Z\right)=70.84 \\
\operatorname{BR}\left(\widetilde{\chi}_{3}^{0} \rightarrow \widetilde{\chi}_{1}^{0} h\right)=29.16 \\
\operatorname{BR}\left(\widetilde{\chi}_{1}^{ \pm} \rightarrow \widetilde{\chi}_{1}^{0} W^{ \pm}\right)=100\end{array}$ & $\begin{array}{c}g g \rightarrow H(A) \\
b \bar{b} \rightarrow H(A) \\
p p \rightarrow b \bar{b} H(A) \\
4 F b \bar{b} H(\mathrm{NLO}) \\
p p \rightarrow \widetilde{\chi}_{1}^{0} \widetilde{\chi}_{2}^{0} \\
p p \rightarrow \widetilde{\chi}_{2}^{0} \widetilde{\chi}_{2}^{0} \\
p p \rightarrow \widetilde{\chi}_{1}^{ \pm} \widetilde{\chi}_{2}^{0} \\
p p \rightarrow \widetilde{\chi}_{1}^{0} \widetilde{\chi}_{3}^{0} \\
p p \rightarrow \widetilde{\chi}_{3}^{0} \widetilde{\chi}_{3}^{0} \\
p p \rightarrow \widetilde{\chi}_{1}^{ \pm} \widetilde{\chi}_{3}^{0} \\
p p \rightarrow \widetilde{\chi}_{1}^{ \pm} \widetilde{\chi}_{1}^{0} \\
p p \rightarrow \widetilde{\chi}_{2}^{0} \widetilde{\chi}_{3}^{0} \\
p p \rightarrow \widetilde{\chi}_{1}^{+} \widetilde{\chi}_{1}^{-}\end{array}$ & $\begin{array}{c}14.76(29.84) \\
111.37(111.86) \\
43.00(43.20) \\
79.30 \\
18.30 \\
4.71 \times 10^{-5} \\
217.90 \\
19.70 \\
9.82 \times 10^{-3} \\
210.20 \\
27.08 \\
107.00 \\
126.00\end{array}$ \\
\hline BP2 & $\begin{array}{c}M_{A}=750 \mathrm{GeV} \\
\tan \beta=12.1 \\
\text { eq. }(4.1)\end{array}$ & $\begin{array}{c}m_{\widetilde{\chi}_{1}^{0}}=3.39 \\
m_{\widetilde{\chi}_{2}^{0}}=251.18 \\
m_{\widetilde{\chi}_{3}^{0}}=255.69 \\
m_{\widetilde{\chi}_{1}^{ \pm}}=248.34 \\
m_{H}=750.22\end{array}$ & $\begin{array}{c}\operatorname{BR}\left(H(A) \rightarrow \widetilde{\chi}_{1}^{0} \widetilde{\chi}_{2}^{0}\right)=10.84(15.31) \\
\operatorname{BR}\left(H(A) \rightarrow \widetilde{\chi}_{1}^{0} \widetilde{\chi}_{3}^{0}\right)=14.26(9.43) \\
\operatorname{BR}\left(\widetilde{\chi}_{2}^{0} \rightarrow \widetilde{\chi}_{1}^{0} Z\right)=51.80 \\
\operatorname{BR}\left(\widetilde{\chi}_{2}^{0} \rightarrow \widetilde{\chi}_{1}^{0} h\right)=48.20 \\
\operatorname{BR}\left(\widetilde{\chi}_{3}^{0} \rightarrow \widetilde{\chi}_{1}^{0} Z\right)=70.00 \\
\operatorname{BR}\left(\widetilde{\chi}_{3}^{0} \rightarrow \widetilde{\chi}_{1}^{0} h\right)=30.00 \\
\operatorname{BR}\left(\widetilde{\chi}_{1}^{ \pm} \rightarrow \widetilde{\chi}_{1}^{0} W^{ \pm}\right)=100\end{array}$ & $\begin{array}{c}g g \rightarrow H(A) \\
b \bar{b} \rightarrow H(A) \\
p p \rightarrow b \bar{b} H(A) \\
4 F b \bar{b} H(\mathrm{NLO}) \\
p p \rightarrow \widetilde{\chi}_{1}^{0} \widetilde{\chi}_{2}^{0} \\
p p \rightarrow \widetilde{\chi}_{2}^{0} \widetilde{\chi}_{2}^{0} \\
p p \rightarrow \widetilde{\chi}_{1}^{ \pm} \widetilde{\chi}_{2}^{0} \\
p p \rightarrow \widetilde{\chi}_{1}^{0} \widetilde{\chi}_{3}^{0} \\
p p \rightarrow \widetilde{\chi}_{3}^{0} \widetilde{\chi}_{3}^{0} \\
p p \rightarrow \widetilde{\chi}_{1}^{ \pm} \widetilde{\chi}_{3}^{0} \\
p p \rightarrow \widetilde{\chi}_{1}^{ \pm} \widetilde{\chi}_{1}^{0} \\
p p \rightarrow \widetilde{\chi}_{2}^{0} \widetilde{\chi}_{3}^{0} \\
p p \rightarrow \widetilde{\chi}_{1}^{+} \widetilde{\chi}_{1}^{-}\end{array}$ & $\begin{array}{c}5.00(12.03) \\
70.21(70.41) \\
22.00(22.06) \\
47.45 \\
17.50 \\
4.72 \times 10^{-5} \\
217.90 \\
19.40 \\
9.85 \times 10^{-3} \\
209.10 \\
26.95 \\
107.00 \\
126.00\end{array}$ \\
\hline
\end{tabular}

Table 3. Two benchmark points to study the mono- $Z+\mathbb{E}_{T}$ and mono- $h+\mathbb{E}_{T}$ final state.

further analyse the b-tag analysis in two different ways. The first is via Santander matching of the $4 \mathrm{~F}$ and $5 \mathrm{~F} b \bar{b} H$ production and the second is by generating the NLO $4 F b \bar{b} H$ production. We describe these two procedures in the following.

In case of the $4 \mathrm{~F}$ and $5 \mathrm{~F}$ matching, we generate the heavy Higgs signal events i.e. $p p \rightarrow H \rightarrow \widetilde{\chi}_{1}^{0} \widetilde{\chi}_{2}^{0}$ and $p p \rightarrow H \rightarrow \widetilde{\chi}_{1}^{0} \widetilde{\chi}_{3}^{0}$ with $\widetilde{\chi}_{2,3}^{0}$ decaying via $\widetilde{\chi}_{2,3}^{0} \rightarrow \widetilde{\chi}_{1}^{0}+Z, Z \rightarrow \ell \ell$ and $\widetilde{\chi}_{2,3}^{0} \rightarrow \widetilde{\chi}_{1}^{0}+h,(h \rightarrow b \bar{b}$ and $h \rightarrow \gamma \gamma)$, in three different production modes separately, namely, the gluon-gluon fusion (diagram (a) in figure 13), bbH fusion process in $4 \mathrm{~F}$ scheme and the $5 \mathrm{~F}$ scheme in the remaining figures. It is important to carefully generate and match the cross sections in the 4 flavour $(4 \mathrm{~F})$ and five flavour $(5 \mathrm{~F})$ schemes. The reason is as follows. The cross section in the $4 \mathrm{~F}$ scheme at leading order (LO), receives contribution from two QCD processes, viz. gluon fusion production, $g g \rightarrow b \bar{b} H$ and quark anti-quark annihilation, $q \bar{q} \rightarrow b \bar{b} H$ (diagram (b) and (c) respectively in figure 13). The heavy Higgs production in both of the cases are accompanied by two $b$-quarks. However, in case of collinear splitting of a gluon into two bottom quarks, the logarithmic terms in the $4 \mathrm{~F}$ 
inclusive cross section becomes very large which has the form of $\sim \ln \left(\frac{\mu_{F}}{m_{b}}\right)$, where $\mu_{F}$ is the factorisation scale. Once these large logarithmic terms are absorbed in the parton distribution function (PDF) of the bottom quark, the theory remains perturbative. This is done by the re-summation of these terms at all orders in the perturbation theory which makes the basis of $5 \mathrm{~F}$ scheme. The heavy Higgs is produced in the $5 \mathrm{~F}$ scheme at LO mainly via the QCD process, $b \bar{b} \rightarrow H$ (diagram (d) in figure 13) with no extra parton in the final state. The processes where heavy Higgs is produced with a quark or gluon, become important when we demand an additional b-jets in the final state along with the heavy Higgs decay products, e.g, $g b \rightarrow b H$ and $b \bar{b} \rightarrow g H$ (diagram (e) and (f) in figure 13). Also, the process $g g \rightarrow b \bar{b} H$ is the LO process in the $4 \mathrm{~F}$ scheme which shows up in $5 \mathrm{~F}$ scheme at the NNLO order. The cross section in these two scheme does not match when calculated upto a fixed order because the perturbative expansion is different in the two schemes. The cross section in the $4 \mathrm{~F}$ scheme is known up to NLO accuracy in QCD [140-143]. On the other hand, the $5 \mathrm{~F}$ scheme calculation is available up to NNLO in QCD [144]. The processes $g b \rightarrow b H$ and $b \bar{b} \rightarrow g H$ has been derived up to NLO order in QCD [145] and the electroweak (EW) [146]. Matching the two schemes thus removes the potentially overlapping part of the cross section and accounts for genuine bottom PDF inside the proton.

The inclusive cross-section in the two schemes agrees very well with an appropriate choice of factorisation and renormalisation scale [147-149]. The cross-section in these two schemes are multiplied by their respective weight factor and added together to get the total inclusive cross-section of the $b \bar{b} H$ process. This is known as Santander matching [150]. The weight factors for such procedure depend logarithmically on the heavy Higgs mass $\left(m_{H}\right)$ and the bottom quark mass $\left(m_{b}\right) .{ }^{9}$ The matched cross-section is given by:

$$
\sigma^{\text {matched }}=\frac{\sigma^{4 \mathrm{FS}}+w \sigma^{5 \mathrm{FS}}}{1+w}
$$

where the $\sigma^{4 \mathrm{FS}}$ and $\sigma^{5 \mathrm{FS}}$ are the cross sections in the $4 \mathrm{~F}$ and $5 \mathrm{~F}$ scheme respectively, and the weight factor $w$, is defined as:

$$
w=\ln \frac{m_{H}}{m_{b}}-2 .
$$

We use MadGraph-2.6.5 [151] to generate the signal and SM, susy background events at tree level (LO). For showering and hadronisation of the signal and background events, we use Pythia-8 [124] with CTEQ611 PDF. We furthermore process the events through Delphes-3.4.1 [137] to take into account the detector effects. We use the default ATLAS card with updated b-tagging efficiency and mis-tagging efficiency of a light or c-jet as a b-jet as a function of the jet transverse momentum, $p_{T}$ [152]. Jets are reconstructed using FastJet-3.2.1 [153] with anti-kt algorithm with $\Delta R=0.4$. The NNLO cross-section for the signal production from gluon fusion and $b \bar{b} H$ process in $5 \mathrm{~F}$ scheme are calculated using SusHi-1.6.1 [135], which calculates Higgs cross sections in gluon fusion and bottom-quark

\footnotetext{
${ }^{9}$ The pole mass of the bottom quark, $m_{b}=4.78 \mathrm{GeV}$ is used which enters in the logarithmic terms during re-summation.
} 

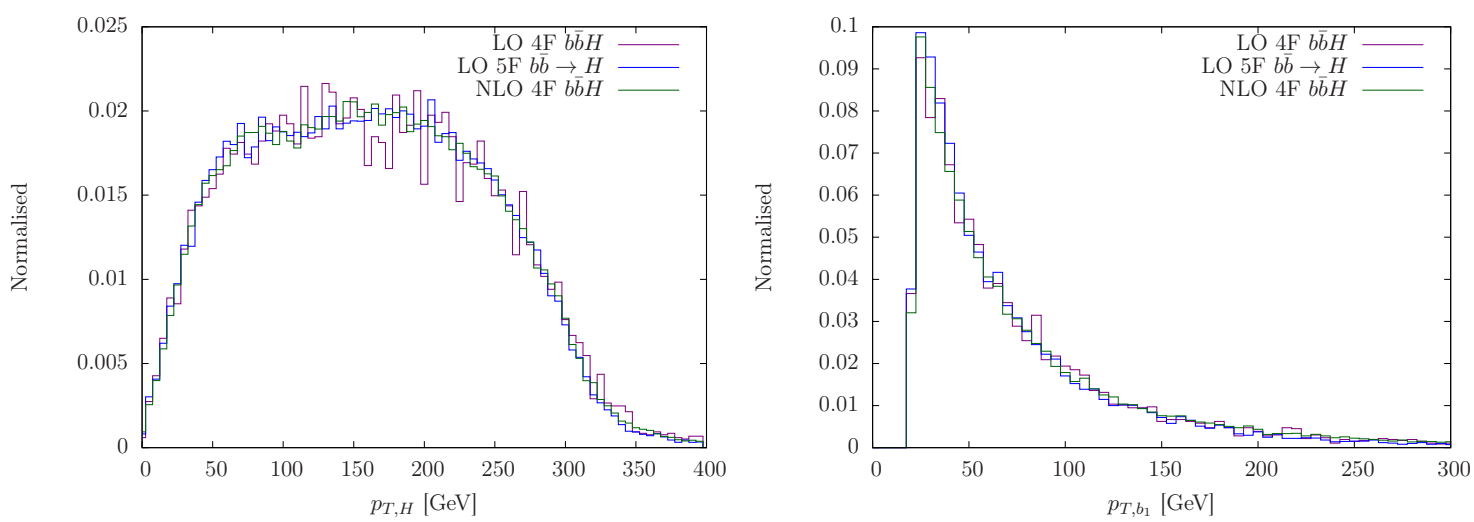

Figure 15. The normalised distributions of $p_{T, H}$ and $p_{T, b_{1}}$ for $m_{H}=650 \mathrm{GeV}$. The plots represent a comparison between $\mathrm{LO} 4 \mathrm{~F}, 5 \mathrm{~F} b \bar{b} H$ and NLO $4 \mathrm{~F} b \bar{b} H$ process.

annihilation at hadron colliders in the SM and various BSM models. It is important to note that SusHi does not generate events, but only predicts differential or integrated cross section upon user request. We thus use these cross sections for normalisation purposes only. Furthermore, as described above, we take care of matching between $4 \mathrm{~F}$ and $5 \mathrm{~F}$ scheme using Santander matching. For the $4 \mathrm{~F}$ scheme $b \bar{b} H$ process we use the cross section at LO from Madgraph. ${ }^{10}$ We use Prospino2 [154] to calculate the cross section of the susy backgrounds at NLO. In table 3, we show the production cross section and the branching ratio of the relevant susy final states. In principle, there are other susy processes viz. $p p \rightarrow \widetilde{\chi}_{1}^{ \pm} \widetilde{\chi}_{4}^{0}, \widetilde{\chi}_{1}^{ \pm} \widetilde{\chi}_{1}^{\mp}, \widetilde{\chi}_{2}^{ \pm} \widetilde{\chi}_{2}^{\mp}$, which can also contribute to the background, however owing to the negligible production cross section and branching ratios for these processes, we exclude them from our analysis.

In case of b-tag analysis, since we generate the $5 F b \bar{b} \rightarrow H$ process at tree level (LO), the extra b-jet comes from the parton shower which may not be in the hard regime. To simulate the kinematic effects of such hard b-tagged jet in the final state, we also perform an analogous analysis by generating the $4 F b \bar{b} H$ process at next-to-leading order (NLO) without merging the $4 \mathrm{~F}$ and $5 \mathrm{~F} b \bar{b} H$ production. We closely follow [155] to generate the NLO $4 F b \bar{b} H$ process for our benchmark points. In figure 15, we compare the kinematic distributions of Higgs boson and the hardest b-jet $p_{T}$ in the LO $4 \mathrm{~F}$ and $5 \mathrm{~F} b \bar{b} H$ process, and also the NLO $4 F b \bar{b} H$ production. These distributions do not change for $4 \mathrm{~F}$ and $5 \mathrm{~F}$ $b \bar{b} H$ process, and the NLO $4 F b \bar{b} H$ production, in the kinematic region which corresponds to our signal region. ${ }^{11}$

\footnotetext{
${ }^{10}$ The cross section in the $4 \mathrm{~F}$ scheme of $b \bar{b} H$ process is calculated in MSSM via Madgraph by using the SLHA file corresponding to the benchmark point as parameter card.

${ }^{11}$ To validate our signal generation, we have also cross-checked the kinematic distributions of $p_{T, h}$ and $p_{T, b_{1}}$ for the Standard Model (SM) $125 \mathrm{GeV}$ Higgs boson. The differences in kinematic distributions for b-jet $p_{T}$ obtained via $4 \mathrm{~F} \mathrm{LO}$ and $4 \mathrm{~F}$ NLO samples seem to decrease with the increasing mass of the Higgs boson.
} 


\subsection{The mono-Z final state}

In this section, we analyse the di-lepton $+\mathbb{E}_{T}$ channel coming from the mono-Z final state viz. $p p \rightarrow H \rightarrow \widetilde{\chi}_{1}^{0}+\widetilde{\chi}_{2,3}^{0} \rightarrow \widetilde{\chi}_{1}^{0}+\left(\widetilde{\chi}_{1}^{0}+Z, Z \rightarrow \ell \ell\right)$ in the context of the future HL-LHC run with center of mass energy, $\sqrt{s}=14 \mathrm{TeV}$ and an integrated luminosity of $3 \mathrm{ab}^{-1}$. Here, $\ell$ refers to electron, muon and tau.

\subsubsection{The $\ell \ell+E_{T}$ channel}

We have discussed at the beginning of the collider analysis that our proposed signal needs to be discriminated against the SM as well as susy background processes. We therefore generate the following six susy backgrounds depending upon the production cross-section as given in table 3 , viz. $p p \rightarrow \widetilde{\chi}_{1}^{0} \widetilde{\chi}_{2,3}^{0}, \widetilde{\chi}_{1}^{ \pm} \widetilde{\chi}_{2,3}^{0}, \quad \widetilde{\chi}_{1}^{ \pm} \widetilde{\chi}_{1}^{\mp}$ and $\widetilde{\chi}_{2}^{0} \widetilde{\chi}_{3}^{0}$. The $p p \rightarrow \widetilde{\chi}_{1}^{0} \widetilde{\chi}_{2,3}^{0}$ and $p p \rightarrow \widetilde{\chi}_{1}^{ \pm} \widetilde{\chi}_{2,3}^{0}$ backgrounds are generated with $\widetilde{\chi}_{2,3}^{0}$ decaying via $\mathrm{Z}$ boson where leptons contain $e, \mu$ and $\tau .{ }^{12}$ For the $p p \rightarrow \widetilde{\chi}_{1}^{ \pm} \widetilde{\chi}_{1}^{\mp}$, the $\widetilde{\chi}_{1}^{ \pm}$decays to $\mathrm{W}$ boson and LSP. The W boson further decays to lepton $(e, \mu$ and $\tau)$ and a neutrino. In case of $p p \rightarrow \widetilde{\chi}_{2}^{0} \widetilde{\chi}_{3}^{0}$, there are two $\mathrm{Z}$ bosons coming from the neutralinos, where one of them decays into leptons and the other decays into neutrinos which gives rise to di-lepton $+\mathbb{E}_{T}$ final state.

The dominant SM backgrounds contributing to this channel are $Z Z$ and $W Z$. We generate them up to additional 3 jets for the $Z Z$ background and 2 jets for the $W Z$ background, matched via MLM scheme [156]. This extra jet contains gluon, light quarks, c-quark and bottom quark. The next dominant backgrounds are $V V V$ where $V=W, Z$ boson and $t \bar{t} Z$. These backgrounds are generated with no extra jet in the final state. In addition, we simulate the $t \bar{t}$ background where the $W$ bosons coming from top-quark are decayed leptonically. There are other sub-dominant background processes like Drell-Yan production, $W W, t \bar{t} h$ and $t \bar{t} W$. In Drell-Yan production, the leptons are produced from a $\mathrm{Z}$ boson or an off-shell photon, i.e. $p p \rightarrow Z / \gamma^{*} \rightarrow \ell \ell$ where $\ell$ contains electron, muon and tau lepton. (For details see appendix C). We divide our analysis into b-veto and b-tag category in the following.

4.1.1.1 b-veto category. We select events containing exactly two isolated, ${ }^{13}$ same flavour and opposite sign leptons (electron or muon) with transverse momentum, $p_{T, \ell}>$ $20 \mathrm{GeV}$ and pseudorapidity, $\left|\eta_{\ell}\right|<2.47$ (2.5) for electron (muon). We require the invariant mass of dilepton system, $76<m_{\ell \ell}<106,{ }^{14}$ and the di-lepton system should be within the pseudorapidity range of $|\eta|<2.5$. We further veto events containing b-jets $\left(N_{b}=\right.$ Number of b-tagged jets in the final state) with $p_{T}>20 \mathrm{GeV},|\eta|<2.5$. To suppress backgrounds with high jet multiplicity further, we restrict the maximum number of light jets $\left(N_{j}\right)$ with $p_{T, j}>20 \mathrm{GeV},\left|\eta_{j}\right|<4.5$ in an event to be one. These correspond to basic trigger cuts. Next, we define more sophisticated variables over which we optimise our signal and background events.

\footnotetext{
${ }^{12}$ We include $\tau$ while simulating both signal and background processes.

${ }^{13}$ We define an isolated electron (muon) as a lepton candidate where the fraction of energy deposited within a cone of $\Delta R<0.5$ is less than $12 \%(25 \%)$ of the lepton $p_{T}$.

${ }^{14}$ This will reduce the contamination from the backgrounds where leptons come from different sources rather than $\mathrm{Z}$ boson in signal event.
} 
In case of the signal events, the $\widetilde{\chi}_{2,3}^{0}$ comes from the decay of heavy Higgs boson and hence has nontrivial transverse momentum, $p_{T}$. For the benchmarks considered here, the mass difference between $\widetilde{\chi}_{2,3}^{0}$ and the decayed particle, $\widetilde{\chi}_{1}^{0}$ is larger than $m_{Z}$. Therefore, the $Z$ boson from $\tilde{\chi}_{2,3}^{0}$ decay can be boosted, giving rise to collimated leptons with small $\Delta R_{\ell \ell}$. A similar feature appears for the susy backgrounds where $\widetilde{\chi}_{2,3}^{0}$ decays to $Z$ boson and $\widetilde{\chi}_{1}^{0}$, viz. $p p \rightarrow \widetilde{\chi}_{1}^{0} \widetilde{\chi}_{2,3}^{0}, \widetilde{\chi}_{1}^{ \pm} \widetilde{\chi}_{2,3}^{0}$ and $\widetilde{\chi}_{2}^{0} \widetilde{\chi}_{3}^{0}$. Contrary to this observation, for the SM backgrounds, the leptons in the final state are not boosted, viz. in diboson background the $\mathrm{Z}$ bosons are produced at rest. We also apply $\Delta \phi_{\ell \ell, E_{T}}>2.1$ for both the benchmark point, which is the azimuthal angle separation between the di-lepton system and the missing transverse energy as an additional discriminating variable. This variable peaks at around $\pi$ for the signal events where the source of missing energy and the di-lepton system are going backto-back, while it is distributed over the whole region for background events. Additionally, we use a large missing transverse energy cut of $\mathbb{E}_{T}>180$ (210) $\mathrm{GeV}$ for the 1st (2nd) benchmark point.

The final discriminating variable arises from genuine imbalance in the missing energy and visible system distribution created in different signal and background samples. We construct the following kinematic variable, viz. $\xi$. We define $\xi$ as,

$$
\xi=\frac{\left|p_{T, \ell \ell}-\mathbb{E}_{T}\right|}{p_{T, \ell \ell}},
$$

which is a measure of momentum imbalance in the system. For the signal and susy backgrounds, the di-lepton system is against $\mathbb{E}_{T}$, which leads to $\xi \sim 0$. For the SM backgrounds, the $\mathbb{E}_{T}$ is very small as compared to the transverse momentum of the two lepton system, giving rise to $\xi \sim 1$.

The discriminating power of $\mathbb{E}_{T}$ and $\Delta R_{\ell \ell}$ distributions for the resonant signal production and susy backgrounds was already seen in section 3.2. Normalised distributions for all four variables discussed above $\left(\mathbb{E}_{T}, \xi, \Delta R_{\ell \ell}, \Delta \phi_{\ell \ell, \mathbb{E}_{T}}\right)$ after basic trigger cuts are shown in figure 16. They show the differential distributions of signal and background processes with respect to the corresponding discriminating variable. It can be clearly seen that there are three distinct classes of distributions, one corresponding to SM processes (dashed blue, green, red lines), second corresponding to susy backgrounds (dashed dark green, yellow, black lines) and finally the signal distributions (solid red, purple lines). These three classes of processes have different features in corresponding variables and it shows that an optimised analysis will be capable of discriminating among the three.

Using the observations described before we do a cut-based analysis optimising for $\Delta R_{\ell \ell}$, $\Delta \phi_{\ell \ell, E_{T}}, \mathbb{E}_{T}$ and $\xi$ variables in favour of the signal events. We show the optimised cuts chosen for the two benchmark points in table 4 along with the applied fixed cuts. We would like to mention here that the cuts obtained after optimisation of signal significance for each kind of signal sample, viz. gluon-gluon fusion, $4 F, 5 F$ scheme and NLO $4 F b \bar{b} H$, are similar. In order to speed up the process of optimisation, we use only samples produced using $5 F$ scheme and use the same optimisation for gluon-gluon fusion, $4 F$ scheme and NLO $4 F b \bar{b} H$ production. As the kinematics of the final state is largely independent of the scheme used to produce the Higgs bosons, this approach is justified. In the following, 


\begin{tabular}{|c|c|}
\hline \multicolumn{2}{|c|}{ Selection cuts } \\
\hline BP 1 & BP 2 \\
\hline $\begin{array}{r}2 \ell, \Lambda \\
76.0<m \\
\left|\eta_{\ell \ell}\right| \\
N_{j} \\
\end{array}$ & $\begin{array}{l}=0 \\
\ell<106.0 \\
=2.5 \\
\leq 1\end{array}$ \\
\hline $\begin{array}{c}\Delta R_{\ell \ell}<1.3 \\
\Delta \phi_{\ell \ell, E_{T}}>2.1 \\
\mathscr{E}_{T}>180 \mathrm{GeV} \\
\xi<0.4\end{array}$ & $\begin{array}{c}\Delta R_{\ell \ell}<1.5 \\
\Delta \phi_{\ell \ell, E_{T}}>2.1 \\
\mathbb{E}_{T}>210 \mathrm{GeV} \\
\xi<0.3\end{array}$ \\
\hline
\end{tabular}

Table 4. The optimised selection cuts for the cut-based analysis in the b-veto category of $\ell \ell+$ $\mathbb{E}_{T}$ channel.
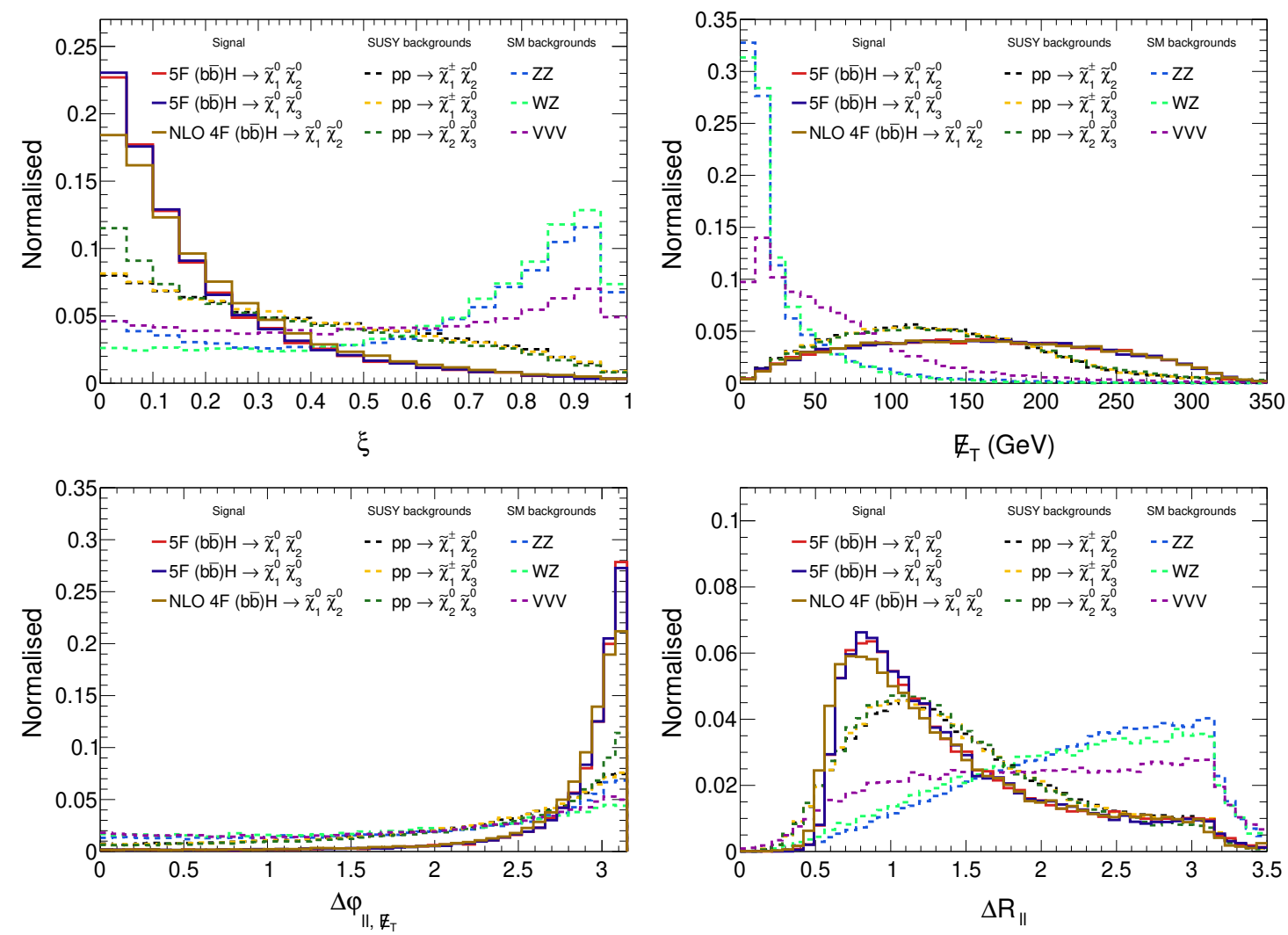

Figure 16. The normalised distributions of $\xi, \mathbb{E}_{T}$, (top panel) $\Delta \phi_{\ell \ell, \mathbb{E}_{T}}$ and $\Delta R_{\ell \ell}$ (bottom panel) for the b-veto category of $\ell \ell+\mathbb{E}_{T}$ final state, after the basic trigger cuts corresponding to BP-1 scenario. We show the signal distributions for $5 \mathrm{~F} b \bar{b} \rightarrow H$ in $\widetilde{\chi}_{1}^{0} \widetilde{\chi}_{2}^{0}$ and $\widetilde{\chi}_{1}^{0} \widetilde{\chi}_{3}^{0}$ final state, and NLO $4 \mathrm{~F}$ $b \bar{b} H$ production in $\widetilde{\chi}_{1}^{0} \widetilde{\chi}_{2}^{0}$ final state, in solid line. Also, we show the susy as well as SM backgrounds in dashed line. 
we present our analysis in two parts, first, the matched $4 \mathrm{~F}, 5 \mathrm{~F} b \bar{b} H$ scheme and second, NLO $4 F b \bar{b} H$ signal process. The final significance in case of matched $4 \mathrm{~F}, 5 \mathrm{~F} b \bar{b} H$ signal is computed by adding the correctly calculated matched $4 F+5 F$ scheme cross section with gluon-gluon fusion cross section. For NLO $4 F b \bar{b} H$ signal, we add the NLO $4 F b \bar{b} H$ cross section with the gluon fusion cross section to get the final signal significance. We show the cut flow table with $5 F b \bar{b} \rightarrow H$ signal production along with the dominant backgrounds in table 5 after applying all the cuts in sequence. This demonstrates relative importance of each cut in reducing the backgrounds. In table 6, we display the susy and SM background yields at the HL-LHC after the cut-based analysis. Here, we have checked that the sub-dominant backgrounds have negligible contribution and therefore neglect them in our final analysis. It is to be noted that the susy backgrounds contribute $\sim 10 \%$ to the total background events. The number of signal events for the gluon fusion production, matched $b \bar{b} H$ process and the NLO $4 F b \bar{b} H$ production at $\sqrt{s}=14 \mathrm{TeV}$ with $3 \mathrm{ab}^{-1}$ of integrated luminosity after the cut-based analysis are shown in table 7 and table 8 . Finally, we calculate the statistical significance as $S / \sqrt{B}$ where $\mathrm{S}$ is the signal yield which is defined as $\sigma\left(p p \rightarrow H \rightarrow \widetilde{\chi}_{1}^{0} \widetilde{\chi}_{2}^{0}\right) \times B R\left(\widetilde{\chi}_{2}^{0} \rightarrow \widetilde{\chi}_{1}^{0} Z\right) \times B R(Z \rightarrow \ell \ell) \times \mathcal{L} \times \epsilon$ for the process $p p \rightarrow H \rightarrow \widetilde{\chi}_{1}^{0} \widetilde{\chi}_{2}^{0}, \widetilde{\chi}_{2}^{0} \rightarrow \widetilde{\chi}_{1}^{0} Z$ where $\epsilon$ is the signal efficiency and $\mathcal{L}$ is the integrated luminosity. Similarly, $B$ represents the total background yield after the cut-based analysis. We quote the final signal significance in table 7 which is 6.57 for the first benchmark point and 4.66 for the second benchmark point in case of $4 \mathrm{~F}, 5 \mathrm{~F}$ matched $b \bar{b} H$ production. However, these significances drop upon adding the systematic uncertainty, ${ }^{15}$ viz. adding a $5 \%$ systematic, the $S / \sqrt{B}$ changes to 1.24 and 1.12 respectively. Therefore, these results are consistent with any extrapolation of the current LHC results. We also tabulate the signal yield from NLO $4 F b \bar{b} H$ process as well as the final signal significance in table 8 . The signal significance is lower as compared to the matched $4 \mathrm{~F}, 5 \mathrm{~F} b \bar{b} H$ analysis which results from the low production cross-section of the NLO $4 F b \bar{b} H$ process. We would like to mention that the final signal efficiencies after the cut-based analysis are similar from the combined $4 \mathrm{~F}, 5 \mathrm{~F} b \bar{b} H$ production and the NLO $4 F b \bar{b} H$ signal process. In case of benchmark point 1 , the final signal efficiency is 0.039 from the NLO $4 F b \bar{b} H$ and 0.038 for the matched $4 \mathrm{~F}, 5 \mathrm{~F}$ $b \bar{b} H$ production. The signal efficiencies are 0.036 and 0.04 from the NLO $4 F b \bar{b} H$ process and matched $4 \mathrm{~F}, 5 \mathrm{~F} b \bar{b} H$ production for benchmark point 2 respectively.

4.1.1.2 b-tag category The event selection in this case contains at least one b-tagged jet along with the two isolated leptons in the final state. We perform the cut-based analysis in a similar way as discussed in the previous section and show the selection cuts in table 9 . The signal and background yields after the cut-based analysis is tabulated in table 10 and table 11. The signal significance improves here over the b-veto analysis due to reduced background composition resulting from the extra b-jet requirement. The signal significance for the benchmark point 1 is $10.48 \sigma$ from matched $4 \mathrm{~F}, 5 \mathrm{~F} b \bar{b} H$ signal and $7.17 \sigma$ from the NLO $4 F b \bar{b} H$ production, without any systematic uncertainty. This large $10.48 \sigma$ and $7.17 \sigma$ significance reduces to $6.80 \sigma$ and $4.65 \sigma$ after consideration of $5 \%$ systematic uncertainty

\footnotetext{
${ }^{15}$ The signal significance formula changes with adding $x \%$ systematic uncertainty: $S / \sqrt{B+(0.01 \times x \times B)^{2}}$.
} 


\begin{tabular}{|c|c|c|c|c|c|c|c|c|c|c|}
\hline \multirow{3}{*}{ Cut flow } & \multicolumn{10}{|c|}{ Event yield with $3 \mathrm{ab}^{-1}$ of integrated luminosity } \\
\hline & \multirow{2}{*}{$\begin{array}{c}\text { Signal (BP 1) } \\
b \bar{b} \rightarrow H(5 F)\end{array}$} & \multicolumn{5}{|c|}{ susy Backgrounds } & \multicolumn{4}{|c|}{ SM Backgrounds } \\
\hline & & $\tilde{\chi}_{1}^{ \pm} \tilde{\chi}_{2}^{0}$ & $\tilde{\chi}_{1}^{ \pm} \widetilde{\chi}_{3}^{0}$ & $\tilde{\chi}_{2}^{0} \widetilde{\chi}_{3}^{0}$ & $\widetilde{\chi}_{1}^{0} \widetilde{\chi}_{2}^{0}$ & $\widetilde{\chi}_{1}^{0} \widetilde{\chi}_{3}^{0}$ & $Z Z$ & $W Z$ & $V V V$ & $t \bar{t} Z$ \\
\hline $2 \ell$ & 2496 & 76885 & 10433 & 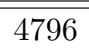 & 7743 & 1124 & 1103323 & 2067690 & 16251 & 12885 \\
\hline$m_{\ell \ell}$ & 2391 & 7036 & 9520 & 4586 & 711 & 1076 & 1084794 & 1952657 & 9626 & 9046 \\
\hline$\left|\eta_{\ell \ell}\right|$ & 2336 & 6829 & 9244 & 4464 & 683 & 1035 & 914415 & 1687769 & 8865 & 8728 \\
\hline$N_{j}$ & 1872 & 2154 & 2946 & 1555 & 570 & 863 & 401278 & 552658 & 1528 & 172 \\
\hline$\Delta R_{\ell \ell}$ & 1064 & 951 & 1332 & 721 & 176 & 280 & 29356 & 28790 & 286 & 48 \\
\hline$\Delta \phi_{\ell \ell, \mathbb{E}_{T}}$ & 1056 & 826 & 1151 & 667 & 172 & 274 & 23326 & 17223 & 234 & 34 \\
\hline $\mathbb{E}_{T}$ & 738 & 390 & 552 & 378 & 78 & 125 & 7620 & 2389 & 98 & 9.5 \\
\hline$\xi$ & 720 & 362 & 515 & 362 & 75 & 121 & 7181 & 2137 & 88 & 8.8 \\
\hline
\end{tabular}

Table 5. The cut-flow table for the benchmark point 1 in the b-veto category of $\ell \ell+\mathbb{E}_{T}$ mode with $5 \mathrm{~F}$ signal production and dominant backgrounds.

\begin{tabular}{|c|c|c|c|c|c|c|c|c|c|c|c|c|}
\hline \multirow[t]{3}{*}{ BPs } & \multicolumn{12}{|c|}{ Background yield at $3 \mathrm{ab}^{-1}$ after all cuts } \\
\hline & \multicolumn{6}{|c|}{ susy Backgrounds } & \multicolumn{5}{|c|}{ SM Backgrounds } & \multirow{3}{*}{$\begin{array}{c}\text { Total } \\
\text { Background }\end{array}$} \\
\hline & $\tilde{\chi}_{1}^{ \pm} \widetilde{\chi}_{2}^{0}$ & $\widetilde{\chi}_{1}^{ \pm} \widetilde{\chi}_{3}^{0}$ & $\widetilde{\chi}_{2}^{0} \widetilde{\chi}_{3}^{0}$ & $\widetilde{\chi}_{1}^{0} \widetilde{\chi}_{2}^{0}$ & $\widetilde{\chi}_{1}^{0} \widetilde{\chi}_{3}^{0}$ & $\tilde{\chi}_{1}^{ \pm} \widetilde{\chi}_{1}^{ \pm}$ & $Z Z$ & $W Z$ & $V V V$ & $t \bar{t} Z$ & $t \bar{t}$ & \\
\hline Order & \multicolumn{6}{|c|}{ NLO [154] } & $\mathrm{LO}$ & $\mathrm{LO}$ & LO & NLO [157] & NNLO [158] & \\
\hline BP 1 & 362 & 515 & 362 & 75 & 121 & 23 & 7181 & 2137 & 88 & 8.8 & $\sim 0$ & 10873 \\
\hline BP 2 & 227 & 305 & 254 & 48 & 73 & 6.8 & 4440 & 1131 & 58 & 5.1 & $\sim 0$ & 6548 \\
\hline
\end{tabular}

Table 6. The background yield at $14 \mathrm{TeV}$ with $3 \mathrm{ab}^{-1}$ of integrated luminosity after the cut-based analysis for the two benchmark points.

\begin{tabular}{|c|c|c|c|c|c|c|c|c|c|}
\hline \multicolumn{10}{|c|}{ Using LO $4 \mathrm{~F}$ and $5 \mathrm{~F} b \bar{b} H$ process } \\
\hline \multirow[t]{3}{*}{ BPs } & \multicolumn{6}{|c|}{ Signal rates at $3 \mathrm{ab}^{-1}$ after all cuts } & \multicolumn{3}{|c|}{ Significance calculation } \\
\hline & \multicolumn{3}{|c|}{$p p \rightarrow H / A \rightarrow \widetilde{\chi}_{1}^{0} \widetilde{\chi}_{2}^{0}$} & \multicolumn{3}{|c|}{$p p \rightarrow H / A \rightarrow \widetilde{\chi}_{1}^{0} \widetilde{\chi}_{3}^{0}$} & \multirow{3}{*}{$\begin{array}{c}\text { Total signal, } \\
\begin{array}{c}S=\frac{N_{4 F}+w N_{5 F}}{1+w} \\
+N_{g g F}\end{array}\end{array}$} & \multirow{3}{*}{$\begin{array}{c}\text { Total background, } \\
\text { B } \\
\text { (From table 6) }\end{array}$} & \multirow{3}{*}{$\begin{array}{c}\text { Significance, } \frac{S}{\sqrt{B}} \\
\text { without (with } 5 \% \text { ) } \\
\text { systematics }\end{array}$} \\
\hline & $\begin{array}{c}p p \rightarrow b \bar{b} H \\
(4 F)\end{array}$ & $\begin{array}{c}b \bar{b} \rightarrow H \\
(5 F)\end{array}$ & $\begin{array}{c}g g \rightarrow H \\
(g g F)\end{array}$ & $\begin{array}{c}p p \rightarrow b \bar{b} H \\
(4 F)\end{array}$ & $\begin{array}{c}b \bar{b} \rightarrow H \\
(5 F)\end{array}$ & $\begin{array}{c}g g \rightarrow H \\
(g g F)\end{array}$ & & & \\
\hline Order & LO & NNLC & {$[135]$} & LO & NNLO & {$[135]$} & & & \\
\hline BP 1 & 55 & 312 & 55 & 73 & \begin{tabular}{|l|l|l}
408 \\
\end{tabular} & 61 & 685 & 10873 & $6.57(1.24)$ \\
\hline BP 2 & 22 & 194 & 20 & 30 & 236 & 20 & 377 & 6548 & $4.66(1.12)$ \\
\hline
\end{tabular}

Table 7. The signal yield along with signal significance for the b-veto category of $\ell \ell+\not E_{T}$ final state. $N_{g g F}, N_{4 F}, N_{5 F}$ are the total event yield from the gluon fusion, $4 F, 5 F$ production processes respectively. The $N_{4 F}$ and $N_{5 F}$ are later added according to the Santander matching as described in the text.

respectively. There are two important points to be noted here. First, this reduction in significance demonstrates the importance of including systematic uncertainty. Second, demanding additional b-jet activity can improve the signal to background discrimination. Also, the final signal efficiencies in case of BP1 (BP2) after applying all the cuts are 0.022 (0.027) from NLO $4 F b \bar{b} H$ process and 0.016 (0.022) from the cross-section weighted sum of the matched $4 F$ and $5 F b \bar{b} H$ production. 


\begin{tabular}{|c|c|c|c|c|c|c|c|}
\hline \multicolumn{8}{|c|}{ Using NLO $4 \mathrm{~F} b \bar{b} H$ process } \\
\hline \multirow[t]{3}{*}{ BPs } & \multicolumn{4}{|c|}{ Signal rates at $3 \mathrm{ab}^{-1}$ after all cuts } & \multicolumn{3}{|c|}{ Significance calculation } \\
\hline & \multicolumn{2}{|c|}{$p p \rightarrow H / A \rightarrow \widetilde{\chi}_{1}^{0} \widetilde{\chi}_{2}^{0}$} & \multicolumn{2}{|c|}{$p p \rightarrow H / A \rightarrow \widetilde{\chi}_{1}^{0} \tilde{\chi}_{3}^{0}$} & \multirow{3}{*}{$\begin{array}{c}\text { Total signal, } \\
S= \\
N_{4 F}^{N L O}+N_{g g F}\end{array}$} & \multirow{3}{*}{$\begin{array}{c}\text { Total background, } \\
\text { B } \\
\text { (From table } 6 \text { ) }\end{array}$} & \multirow{3}{*}{$\begin{array}{c}\text { Significance, } \frac{S}{\sqrt{B}} \\
\text { without (with } 5 \% \text { ) } \\
\text { systematics }\end{array}$} \\
\hline & $\begin{array}{c}p p \rightarrow b \bar{b} H \\
(4 F)\end{array}$ & $\begin{array}{c}g g \rightarrow H \\
(g g F)\end{array}$ & $\begin{array}{c}p p \rightarrow b \bar{b} H \\
(4 F)\end{array}$ & $\begin{array}{c}g g \rightarrow H \\
(g g F)\end{array}$ & & & \\
\hline Order & NLO [159] & NNLO [135] & NLO [159] & NNLO [135] & & & \\
\hline BP 1 & 130 & 55 & 164 & 61 & 410 & 10873 & $3.93(0.74)$ \\
\hline BP 2 & 72 & 20 & 91 & 20 & 203 & 6548 & $2.51(0.6)$ \\
\hline
\end{tabular}

Table 8. The signal yield along with signal significance for the b-veto category of $\ell \ell+\mathbb{E}_{T}$ final state for NLO $4 F b \bar{b} H$ process. $N_{g g F}, N_{4 F}^{N L O}$ are the total event yield from the gluon fusion NLO $4 F b \bar{b} H$ production processes respectively.

\begin{tabular}{|r|c|}
\hline \multicolumn{2}{|c|}{ Selection cuts } \\
\hline BP 1 & BP 2 \\
\hline \multicolumn{2}{|c|}{$2 \ell, N_{b} \geq 1$} \\
$76.0<m_{\ell \ell}<106.0$ \\
$\left|\eta_{\ell \ell}\right|<2.5$ \\
$N_{j} \leq 1$ \\
\hline$\Delta R_{\ell \ell}<1.3$ & $\Delta R_{\ell \ell}<1.3$ \\
$\Delta \phi_{\ell \ell, E_{T}}>2.1$ & $\Delta \phi_{\ell \ell, \mathbb{E}_{T}}>2.3$ \\
$\mathbb{E}_{T}>160 \mathrm{GeV}$ & $\mathbb{E}_{T}>170 \mathrm{GeV}$ \\
$\xi<0.4$ & $\xi<0.8$ \\
\hline
\end{tabular}

Table 9. The optimised selection cuts for the cut-based analysis in the b-tag category of $\ell \ell+$ $\mathbb{E}_{T}$ channel.

\begin{tabular}{|c|c|c|c|c|c|c|c|c|c|c|c|c|}
\hline \multirow[t]{3}{*}{$\mathrm{BPs}$} & \multicolumn{12}{|c|}{ Background yield at $3 \mathrm{ab}^{-1}$ after all cuts } \\
\hline & \multicolumn{6}{|c|}{ susy Backgrounds } & \multicolumn{5}{|c|}{ SM Backgrounds } & \multirow{2}{*}{$\begin{array}{c}\text { Total } \\
\text { Background }\end{array}$} \\
\hline & $\widetilde{\chi}_{1}^{ \pm} \widetilde{\chi}_{2}^{0}$ & $\tilde{\chi}_{1}^{ \pm} \tilde{\chi}_{3}^{0}$ & $\tilde{\chi}_{2}^{0} \widetilde{\chi}_{3}^{0}$ & $\tilde{\chi}_{1}^{0} \widetilde{\chi}_{2}^{0}$ & $\tilde{\chi}_{1}^{0} \tilde{\chi}_{3}^{0}$ & $\tilde{\chi}_{1}^{ \pm} \tilde{\chi}_{1}^{ \pm}$ & $Z Z$ & $W Z$ & $V V V$ & $t \bar{t} Z$ & $t \bar{t}$ & \\
\hline BP 1 & 29.15 & 43.09 & 75.17 & 1.31 & 1.92 & 0.2 & 137.05 & 151.18 & 12.40 & 84.70 & 14.62 & 550.79 \\
\hline BP 2 & 37.28 & 43.23 & 80.16 & 1.46 & 1.89 & $\sim 0$ & 137.05 & 100.79 & 14.80 & 98.57 & 10.96 & 526.19 \\
\hline
\end{tabular}

Table 10. The background yield at $14 \mathrm{TeV}$ with $3 \mathrm{ab}^{-1}$ of integrated luminosity after the cut-based analysis for the two benchmark points in b-tag category.

\subsection{The mono-h final state}

As we have seen in table 3, the electroweakinos can decay to the SM Higgs with a substantial rate, and can potentially be probed via the mono-h final state. The signal processes are $p p \rightarrow H \rightarrow \widetilde{\chi}_{1}^{0}+\widetilde{\chi}_{2,3}^{0} \rightarrow \widetilde{\chi}_{1}^{0}+\left(\widetilde{\chi}_{1}^{0}+h\right)$. Here, we consider two possible decay modes of the SM Higgs for our analysis, viz. $h \rightarrow b \bar{b}$ and $h \rightarrow \gamma \gamma$, which gives rise to $b \bar{b}+\mathbb{E}_{T}$ and $\gamma \gamma+\mathbb{E}_{T}$ final state respectively. The $b \bar{b}+\mathbb{E}_{T}$ channel has substantial rate $(\operatorname{BR}(h \rightarrow b \bar{b}) \sim$ $0.58)$ but this channel is contaminated by huge QCD backgrounds. While the $\gamma \gamma+\mathbb{E}_{T}$ channel suffers from small production rate because of the very small decay rate of $h \rightarrow \gamma \gamma$ $\left(\mathrm{BR}(h \rightarrow \gamma \gamma) \sim 2.27 \times 10^{-3}\right)$ however has the advantage of being clean in terms of the background contamination. In the next two subsections, we do a cut-based analysis for these two channels. 


\begin{tabular}{|c|c|c|c|c|c|c|c|c|c|}
\hline \multirow[t]{3}{*}{ BPs } & \multicolumn{6}{|c|}{ Signal rates at $3 \mathrm{ab}^{-1}$ after all cuts } & \multicolumn{3}{|c|}{ Significance calculation } \\
\hline & \multicolumn{3}{|c|}{$p p \rightarrow H / A \rightarrow \widetilde{\chi}_{1}^{0} \widetilde{\chi}_{2}^{0}$} & \multicolumn{3}{|c|}{$p p \rightarrow H / A \rightarrow \widetilde{\chi}_{1}^{0} \widetilde{\chi}_{3}^{0}$} & \multirow{2}{*}{$\begin{array}{c}\text { Total signal, } \\
\text { S }\end{array}$} & \multirow{2}{*}{$\begin{array}{c}\text { Total background, } \\
\text { B } \\
\text { (From table } 10)\end{array}$} & \multirow{2}{*}{$\begin{array}{c}\text { Significance, } \frac{S}{\sqrt{B}} \\
\text { without (with } 5 \% \text { ) } \\
\text { systematics }\end{array}$} \\
\hline & $4 F$ & $5 F$ & $g g F$ & $4 F$ & $5 F$ & $g g F$ & & & \\
\hline $\mathrm{BP} 1$ & 37.51 & 128.15 & 1.75 & 44.23 & 168.93 & 2.27 & 246.06 & 550.79 & $10.48(6.80)$ \\
\hline BP 2 & 23.22 & 100.89 & 0.91 & 25.31 & 125.28 & 0.86 & 184.14 & 526.19 & $8.03(5.28)$ \\
\hline \multicolumn{10}{|c|}{ Using NLO $4 \mathrm{~F} b \bar{b} H$ process } \\
\hline BPs & \multicolumn{2}{|c|}{$4 F \mathrm{NLO}$} & $g g F$ & \multicolumn{2}{|c|}{$4 F \mathrm{NLO}$} & $g g F$ & $\begin{array}{c}\text { Total signal, } \\
S\end{array}$ & $\begin{array}{c}\text { Total background, } \\
\text { B }\end{array}$ & $\begin{array}{l}\frac{S}{\sqrt{B}} \text { without (with } \\
5 \% \text { ) systematics }\end{array}$ \\
\hline BP 1 & \multicolumn{2}{|c|}{72.43} & 1.75 & \multicolumn{2}{|c|}{91.79} & 2.27 & 168.24 & 550.79 & $7.17(4.65)$ \\
\hline $\mathrm{BP} 2$ & \multicolumn{2}{|c|}{54.2} & 0.91 & \multicolumn{2}{|c|}{67.13} & 0.86 & 123.1 & 526.19 & $5.37(3.53)$ \\
\hline
\end{tabular}

Table 11. The signal yield for two benchmark points along with signal significance for the b-tag category of $\ell \ell+\mathbb{E}_{T}$ final state.

\subsubsection{The $b \bar{b}+\boldsymbol{E}_{T}$ channel}

As with the previous optimisation procedure, there are two kinds of backgrounds to this channel, viz. the backgrounds arising from the SM processes and the susy backgrounds. In case of susy backgrounds, we generate samples associated with large production crosssection (table 3), viz. $p p \rightarrow \widetilde{\chi}_{1}^{0} \widetilde{\chi}_{2,3}^{0}, \tilde{\chi}_{1}^{ \pm} \tilde{\chi}_{2,3}^{0}$ and $\tilde{\chi}_{2}^{0} \tilde{\chi}_{3}^{0}$. Each of the $\tilde{\chi}_{2,3}^{0}$ can decay into a $\mathrm{Z}$ or $\mathrm{h}$ and $\tilde{\chi}_{1}^{ \pm}$decays into a $\mathrm{W}$ boson. Based on this, there could be 5 possible final state configurations, viz. $h h, Z h, Z Z, W h$ and $W Z$. We combine all of these decay configuration while generating this background.

In addition, we generate the dominant irreducible SM backgrounds, viz. $Z b \bar{b}$ and $t \bar{t}$. For the $Z b \bar{b}$, we decay the $Z$ boson to neutrinos to get a similar final state as the signal event. We separately generate the $t \bar{t}$ background in fully leptonic mode, in semi-leptonic mode, and in hadronic mode. We also generate the other subdominant SM backgrounds, i.e. $Z h, W h, t \bar{t} h, t \bar{t} Z$ and $t \bar{t} W$. The $Z h$ background is generated upon merging with two jets in the final state by employing the MLM merging scheme. We generate the $W h$ background by merging with one extra parton in the final state where the SM Higgs is decayed to pair of bottom quarks and the $W$ boson decays leptonically. We also generate the $t \bar{t}+X$ backgrounds where $X=h, Z, W$ with no extra jets in the final state. (See appendix $\mathrm{C}$ for details.)

4.2.1.1 b-veto category. The event selection for this analysis is governed by demanding that the event must have exactly two b-jets with $p_{T}>20 \mathrm{GeV}$ and $|\eta|<2.5$. We veto leptons $\left(N_{\ell}=\right.$ Number of leptons in the final state) with $p_{T}>20 \mathrm{GeV}$ and $|\eta|<2.47$ (2.5) (for $e(\mu)$ ) in the final state to reduce the contamination from $t \bar{t}, W h$ and $p p \rightarrow \widetilde{\chi}_{1}^{ \pm} \widetilde{\chi}_{2,3}^{0}$ backgrounds where the final state contains leptons. The light jets are required to satisfy the transverse momentum of $p_{T}>20 \mathrm{GeV}$ and pseudorapidity, $|\eta|<4.5$. Finally, we construct the kinematic variables to perform the cut-based analysis. The invariant mass of the bottom pair will peak around the SM Higgs mass and we require $90<m_{b b}<130 \mathrm{GeV}$ [160]. We further require at most one light jet in the final state. These cuts define the set of basic trigger cuts in this analysis. The separation in the $\eta-\phi$ plane between the two b-jets measured as $\Delta R_{b b}$ is small for the signal event since the b-jets coming from SM Higgs 


\begin{tabular}{|c|c|}
\hline \multicolumn{2}{|c|}{ Selection cuts } \\
\hline BP 1 & BP 2 \\
\hline 2 b-jet, $N_{\ell}=0$ \\
$90.0<m_{b b}<130.0$ \\
$N_{j} \leq 1$ \\
\hline $0.4<\Delta R_{b b}<1.4$ & $0.4<\Delta R_{b b}<1.3$ \\
$\Delta \phi_{b b, \mathbb{E}_{T}}>2.8$ & $\Delta \phi_{b b, \mathbb{E}_{T}}>2.6$ \\
$\mathbb{E}_{T}>180 \mathrm{GeV}$ & $\mathbb{E}_{T}>210 \mathrm{GeV}$ \\
\hline
\end{tabular}

Table 12. The selection cuts optimised in the $b \bar{b}+\mathbb{E}_{T}$ channel for the cut-based analysis.
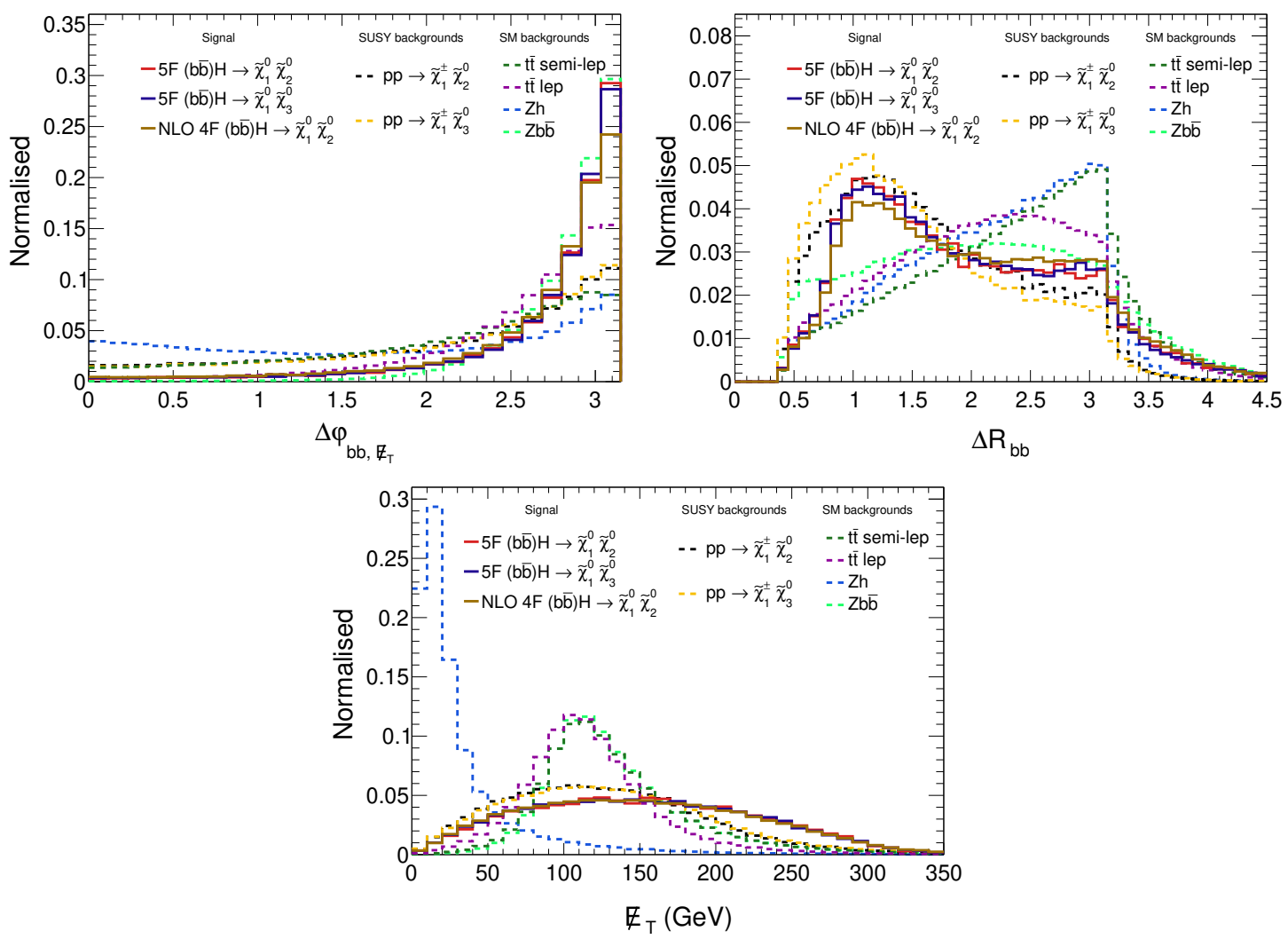

Figure 17. The normalised distributions of $\Delta \phi_{b b, \mathbb{E}_{T}}, \Delta R_{b b}$ (top panel) and $\mathbb{E}_{T}$ (bottom panel) for the $b \bar{b}+\mathbb{E}_{T}$ final state after the basic trigger cuts for the BP 1 scenario.

are boosted, while this is not the case for the SM backgrounds. The missing transverse energy, $\mathbb{E}_{T}$ is large for the signal event as compared to the backgrounds as discussed previously (see section 4.1). Also, we construct the azimuthal angle separation, between the missing transverse momentum, $\mathbb{E}_{T}$ and the two b-jet system, viz. $\Delta \phi_{b \bar{b}, \mathbb{E}_{T}}$. We show the normalised distributions of these kinematic variables after trigger cuts for the signal and the dominant backgrounds in figure 17 . It can be seen that $\mathbb{E}_{T}$ and $\Delta R_{b b}$ are very strong discriminating variables between the signal and background processes. 


\begin{tabular}{|c|c|c|c|c|c|c|c|c|c|c|}
\hline \multirow{3}{*}{ Cut flow } & \multicolumn{10}{|c|}{ Event yield with $3 \mathrm{ab}^{-1}$ of integrated luminosity } \\
\hline & \multirow{2}{*}{$\begin{array}{c}\text { Signal } \\
b \bar{b} \rightarrow H(5 F)\end{array}$} & \multicolumn{5}{|c|}{ susy Backgrounds } & \multicolumn{4}{|c|}{ SM Backgrounds } \\
\hline & & $\tilde{\chi}_{1}^{ \pm} \tilde{\chi}_{2}^{0}$ & $\widetilde{\chi}_{1}^{ \pm} \widetilde{\chi}_{3}^{0}$ & $\widetilde{\chi}_{2}^{0} \widetilde{\chi}_{3}^{0}$ & $\widetilde{\chi}_{1}^{0} \widetilde{\chi}_{2}^{0}$ & $\widetilde{\chi}_{1}^{0} \widetilde{\chi}_{3}^{0}$ & $t \bar{t}$ semi-lep & $t \bar{t}$ lep & $Z b \bar{b}$ & $Z h$ \\
\hline 2 b-jet & 13462 & 52229 & 38069 & 24940 & 4249 & 3440 & 20986447 & 6550973 & 1819565 & 401788 \\
\hline$m_{b b}$ & 6157 & 24030 & 14348 & 9650 & 2077 & 1335 & 4703884 & 1395299 & 330348 & 207187 \\
\hline$N_{j}$ & 4468 & 7433 & 4317 & 3808 & 1687 & 1081 & 438444 & 665118 & 233317 & 86475 \\
\hline$\Delta R_{b b}$ & 1861 & 2427 & 1542 & 1184 & 350 & 264 & 40719 & 41208 & 37335 & 7195 \\
\hline$\Delta \phi_{b b, \mathbb{E}_{T}}$ & 1728 & 1518 & 959 & 895 & 312 & 231 & 27123 & 24511 & 32073 & 4839 \\
\hline $\mathbb{E}_{T}$ & 1305 & 902 & 575 & 545 & 181 & 130 & 7229 & 1873 & 13758 & 2543 \\
\hline
\end{tabular}

Table 13. The cut-flow table for the benchmark point 1 in the $b \bar{b}+\mathbb{E}_{T}$ mode with $5 \mathrm{~F}$ signal production and dominant backgrounds.

\begin{tabular}{|c|c|c|c|c|c|c|c|c|c|c|c|c|c|c|c|}
\hline \multirow[t]{3}{*}{ BPs } & \multicolumn{15}{|c|}{ Background yield at $3 \mathrm{ab}^{-1}$ after all cuts } \\
\hline & \multicolumn{5}{|c|}{ susy Backgrounds } & \multicolumn{9}{|c|}{ SM Backgrounds } & \multirow{3}{*}{$\begin{array}{c}\text { Total } \\
\text { Background }\end{array}$} \\
\hline & $\tilde{\chi}_{1}^{ \pm} \widetilde{\chi}_{2}^{0}$ & $\tilde{\chi}_{1}^{ \pm} \widetilde{\chi}_{3}^{0}$ & $\tilde{\chi}_{2}^{0} \tilde{\chi}_{3}^{0}$ & \begin{tabular}{|l|l|}
$\tilde{\chi}_{1}^{0} \widetilde{\chi}_{2}^{0}$ \\
\end{tabular} & $\widetilde{\chi}_{1}^{0} \widetilde{\chi}_{3}^{0}$ & $t \bar{t}$ had & $t \bar{t}$ semi-lep & $t \bar{t}$ lep & $Z b \bar{b}$ & $Z h$ & $W h$ & $t \bar{t} h$ & $t \bar{t} Z$ & $t \bar{t} W$ & \\
\hline Order & \multicolumn{5}{|c|}{ NLO [154] } & \multicolumn{3}{|c|}{ NNLO [158] } & LO & \multicolumn{2}{|c|}{$\begin{array}{c}\text { NNLO (QCD)+ } \\
\text { NLO (EW) [161] } \\
\end{array}$} & NLO $[161]$ & NLO $[157]$ & NLO [162] & \\
\hline BP 1 & 902 & 575 & 545 & 181 & 130 & $\sim 0$ & 7229 & 1873 & 13758 & 2543 & 19 & 32 & 98 & 28 & 27913 \\
\hline BP 2 & 552 & 408 & 376 & 111 & 88 & $\sim 0$ & 3439 & 525 & 7768 & 1683 & 12 & 24 & 65 & 18 & 15069 \\
\hline
\end{tabular}

Table 14. The background yield at $14 \mathrm{TeV}$ with $3 \mathrm{ab}^{-1}$ of integrated luminosity after the cut-based analysis for the two benchmark points.

With the discriminating variables $\left(\Delta R_{b b}, \Delta \phi_{b b, \mathbb{E}_{T}}\right.$ and $\left.\mathbb{E}_{T}\right)$ explained above, we suggest a cut-based analysis optimising the signal over background. The final choice of cuts for these variables are listed in table 12. As explained in the previous section, we use on the $5 F$ scheme sample for performing the optimisation. Corresponding signal and dominant background yields after all the cuts in succession are displayed in table 13. The number of SM and susy background events corresponding to each benchmark points at the HL-LHC configuration are given in table 14. The final signal significance along with the signal yield is presented in table 15 . Here, the signal significance is slightly higher than the previous $\ell \ell+\mathbb{E}_{T}$ analysis (section 4.1.1.1). Since the $S / B$ ratio is very poor in this channel which reduces the significance drastically upon adding a systematic uncertainty, viz. for the 1st benchmark point $S / \sqrt{B}$ changes from 7.25 to 0.86 and from 4.7 to 0.56 by adding a $5 \%$ systematic, in case of matched $4 \mathrm{~F}, 5 \mathrm{~F} b \bar{b} H$ and NLO $4 F b \bar{b} H$ production respectively.

4.2.1.2 b-tag category. In this channel, we demand at least three b-tagged jets with $p_{T}>20 \mathrm{GeV}$ and $|\eta|<2.5$ in the final state. Similar to the previous b-veto analysis, we reconstruct the kinematic variables for our cut-based analysis. We would like to mention here that the $t \bar{t}$ semi-leptonic background has an increased overlapping distribution in $\Delta R_{b b}, \Delta \phi_{b b, \epsilon_{T}}$ variables with signal $\left(0.4<\Delta R_{b b}<1.4, \Delta \phi_{b b, \mathbb{E}_{T}}>2.4\right)$, making this background dominant, unlike in the b-veto case. We show a comparison of these distributions with the $t \bar{t}$ semi-leptonic background in the b-veto case in figure 18. It is particularly interesting to understand the origin of this increased overlap. As this category demands 3 b-tagged jets, the extra b-jet in the $t \bar{t}$ background in semi-leptonic mode arises when a 


\begin{tabular}{|c|c|c|c|c|c|c|c|c|c|}
\hline \multirow[t]{3}{*}{$\mathrm{BPs}$} & \multicolumn{6}{|c|}{ Signal rates at $3 \mathrm{ab}^{-1}$ after all cuts } & \multicolumn{3}{|c|}{ Significance calculation } \\
\hline & \multicolumn{3}{|c|}{$p p \rightarrow H / A \rightarrow \widetilde{\chi}_{1}^{0} \widetilde{\chi}_{2}^{0}$} & \multicolumn{3}{|c|}{$p p \rightarrow H / A \rightarrow \widetilde{\chi}_{1}^{0} \widetilde{\chi}_{3}^{0}$} & \multirow{2}{*}{$\begin{array}{c}\text { Total signal, } \\
\text { S }\end{array}$} & \multirow{2}{*}{$\begin{array}{c}\text { Total background, } \\
\text { B } \\
\text { (From table } 14 \text { ) }\end{array}$} & \multirow{2}{*}{$\begin{array}{c}\text { Significance, } \frac{S}{\sqrt{B}} \\
\text { without (with } 5 \% \text { ) } \\
\text { systematics }\end{array}$} \\
\hline & $4 F$ & $5 F$ & $g g F$ & $4 F$ & $5 F$ & $g g F$ & & & \\
\hline $\mathrm{BP} 1$ & 137 & 859 & 127 & 73 & 447 & 59 & 1211 & 27913 & $7.25(0.86)$ \\
\hline BP 2 & 74 & 534 & 52 & 41 & 294 & 25 & 729 & 15069 & $4(0.95)$ \\
\hline \multicolumn{10}{|c|}{ Using NLO $4 \mathrm{~F} b \bar{b} H$ process } \\
\hline BPs & \multicolumn{2}{|c|}{$4 F \mathrm{NLO}$} & $g g F$ & \multicolumn{2}{|c|}{$4 F \mathrm{NLO}$} & $g g F$ & $\begin{array}{l}\text { Total signal, } S= \\
N_{4 F}^{N L O}+N_{g g F}\end{array}$ & $\begin{array}{c}\text { Total background, } \\
\text { B } \\
\end{array}$ & $\begin{array}{l}\frac{S}{\sqrt{B}} \text { without (with } \\
5 \% \text { ) systematics }\end{array}$ \\
\hline $\mathrm{BP} 1$ & \multicolumn{2}{|c|}{386} & 127 & \multicolumn{2}{|c|}{212} & 59 & 784 & 27913 & $4.7(0.56)$ \\
\hline BP 2 & \multicolumn{2}{|c|}{233} & 52 & \multicolumn{2}{|c|}{127} & 25 & 437 & 15069 & $3.56(0.57)$ \\
\hline
\end{tabular}

Table 15. The signal yield for two benchmark points along with signal significance for the $b \bar{b}+\mathbb{E}_{T}$ final state.
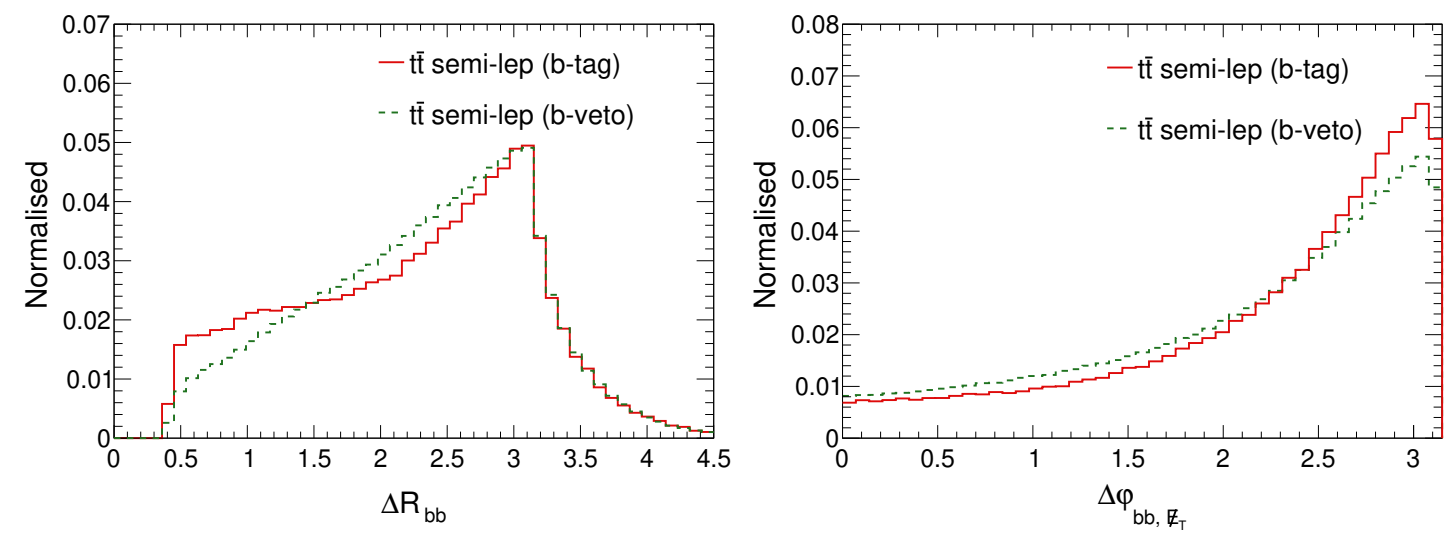

Figure 18. The comparison between the normalised distributions of $\Delta R_{b b}$ (left) and $\Delta \phi_{b b, \mathbb{E}_{T}}$ (right panel) for $t \bar{t}$ semi-leptonic background in the b-veto and b-tag category of $b \bar{b}+\mathbb{E}_{T}$ final state.

c-quark fakes as a b-jet in hadronic decays of $\mathrm{W}$ bosons originating from tops. This fake b-jet can have high transverse momentum and gets selected when reconstructing the two b-jet system. However, we note that there must be a light jet around this di-b-jet system, which originates from the $W$ boson decay for background processes. To catch this feature in our optimisation analysis, we first demand that events must have at most one light jet in the final state. If the events contain a light jet then the di-b-jet system is reconstructed with b-jets closest in the $\eta-\phi$ plane, i.e. we select jets for which $\Delta R_{b b}$ is minimum. Using this $b \bar{b}$ system, we compute the distance between this b-jet system and the light jet, $\Delta R_{b b, j}$, as shown in figure 19. This distribution is shifted towards low values of $\Delta R_{b b, j}$ for the $t \bar{t}$ semi-leptonic than the signal events. Therefore, we include this variable in our cut-based analysis and put a lower bound after optimisation. To construct the other variables, viz. $m_{b b}, \Delta R_{b b}$, and $\Delta \phi_{b b, E_{T}}$, we take the two hardest b-jets in the event as before. We show the optimised selection cuts in table 16 . We quote the background yields and final signal significance along with signal yields after the cut-based analysis in table 17 and table 18 . The tables demonstrate that the final significance is about 4 and 3 for both the benchmark points in case of matched $4 \mathrm{~F}, 5 \mathrm{~F} b \bar{b} H$ and NLO $4 F b \bar{b} H$ production respectively. With 


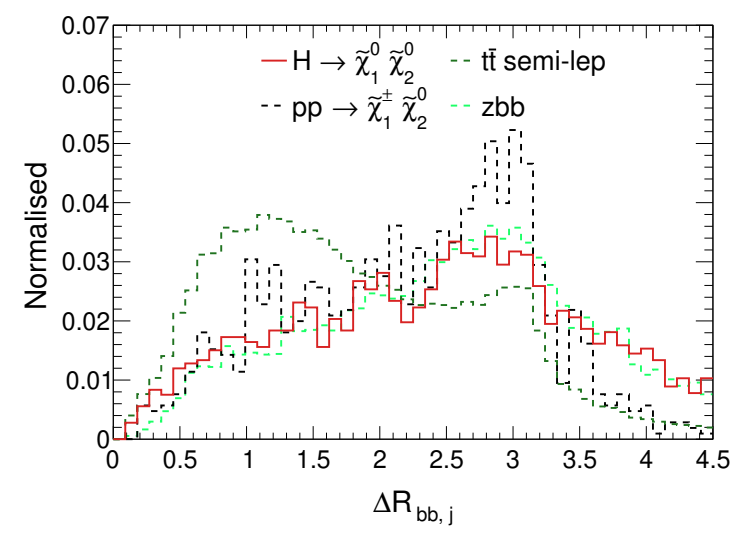

Figure 19. The normalised distributions of $\Delta R_{b b, j}$ for the $t \bar{t}$ semi-leptonic background along with other dominant backgrounds in the b-tag category of $b \bar{b}+\mathbb{E}_{T}$ final state for benchmark point 1 . Overlaid in solid is the distribution for the $5 F$ scheme signal process.

\begin{tabular}{|c|c|}
\hline \multicolumn{2}{|c|}{ Selection cuts } \\
\hline BP 1 & BP 2 \\
\hline \multicolumn{2}{|c|}{$N_{b} \geq 3, N_{\ell}=0$} \\
$N_{j} \leq 1$ \\
$90.0<m_{b b}<130.0$ \\
\hline $0.4<\Delta R_{b b}<1.5$ & $0.4<\Delta R_{b b}<1.4$ \\
$\Delta \phi_{b b, \mathbb{E}_{T}}>2.3$ & $\Delta \phi_{b b,\left.\right|_{T}}>2.2$ \\
$\mathbb{E}_{T}>180 \mathrm{GeV}$ & $\mathbb{E}_{T}>210 \mathrm{GeV}$ \\
$\Delta R_{b b, j}>2.2$ & $\Delta R_{b b, j}>1.7$ \\
\hline
\end{tabular}

Table 16. The selection cuts optimised for the b-tag $b \bar{b}+\mathbb{E}_{T}$ channel for the cut-based analysis.

\begin{tabular}{|c||c|c|c|c|c||c|c|c|c|c|c|c|c|c||c|}
\hline \multicolumn{1}{|c||}{ BPs } & \multicolumn{10}{c|}{ Background yield at $3 \mathrm{ab}^{-1}$ after all cuts } \\
\cline { 2 - 16 } & \multicolumn{10}{c|}{ susy Backgrounds } & \multicolumn{10}{c|}{ Total } \\
& $\tilde{\chi}_{1}^{ \pm} \widetilde{\chi}_{2}^{0}$ & $\widetilde{\chi}_{1}^{ \pm} \widetilde{\chi}_{3}^{0}$ & $\widetilde{\chi}_{2}^{0} \widetilde{\chi}_{3}^{0}$ & $\widetilde{\chi}_{1}^{0} \widetilde{\chi}_{2}^{0}$ & $\widetilde{\chi}_{1}^{0} \widetilde{\chi}_{3}^{0}$ & $t \bar{t}$ had & $t \bar{t}$ semi-lep & $t \bar{t}$ lep & $Z b \bar{b}$ & $Z h$ & $W h$ & $t \bar{t} h$ & $t \bar{t} Z$ & $t \bar{t} W$ & Background \\
\hline \hline BP 1 & 45.16 & 32.31 & 143.41 & 2.79 & 1.64 & $\sim 0$ & 2947.89 & 470.61 & 741.51 & 40.13 & 0.41 & 164.13 & 44.72 & 6.24 & 4640.95 \\
\hline BP 2 & 28.16 & 18.48 & 83.34 & 1.88 & 1.88 & $\sim 0$ & 1784.78 & 235.30 & 527.80 & 34.40 & 0.26 & 58.68 & 43.44 & 4.16 & 2822.56 \\
\hline
\end{tabular}

Table 17. The background yield at $14 \mathrm{TeV}$ with $3 \mathrm{ab}^{-1}$ of integrated luminosity after the cut-based analysis for the two benchmark points in the b-tag category.

respect to b-veto category, we obtain lesser significance for b-tag primarily because of a different background composition.

\subsubsection{The $\gamma \gamma+\boldsymbol{E}_{T}$ channel}

Finally, we turn our focus on the $\gamma \gamma+\mathbb{E}_{T}$ final state which is clean in terms of the background contamination with the disadvantage of having very low event yield as compared to the other search channels discussed earlier. We generate the following susy backgrounds, viz. $p p \rightarrow \widetilde{\chi}_{1}^{0} \widetilde{\chi}_{2,3}^{0}, \widetilde{\chi}_{1}^{ \pm} \widetilde{\chi}_{2,3}^{0}$ and $\widetilde{\chi}_{2}^{0} \widetilde{\chi}_{3}^{0}$. For all of these backgrounds, the $\widetilde{\chi}_{2,3}^{0}$ is decayed to SM Higgs boson and it further decays to a photon pair. The dominant SM backgrounds 


\begin{tabular}{|c|c|c|c|c|c|c|c|c|c|}
\hline \multirow[t]{3}{*}{ BPs } & \multicolumn{6}{|c|}{ Signal rates at $3 \mathrm{ab}^{-1}$ after all cuts } & \multicolumn{3}{|c|}{ Significance calculation } \\
\hline & \multicolumn{3}{|c|}{$p p \rightarrow H / A \rightarrow \widetilde{\chi}_{1}^{0} \widetilde{\chi}_{2}^{0}$} & \multicolumn{3}{|c|}{$p p \rightarrow H / A \rightarrow \widetilde{\chi}_{1}^{0} \widetilde{\chi}_{3}^{0}$} & \multirow{2}{*}{$\begin{array}{c}\text { Total signal, } \\
\mathrm{S}\end{array}$} & \multirow{2}{*}{$\begin{array}{c}\text { Total background, } \\
\text { B } \\
\text { (From table 17) }\end{array}$} & \multirow{2}{*}{$\begin{array}{l}\text { Significance, } \\
\frac{S}{\sqrt{B}}\end{array}$} \\
\hline & $4 F$ & $5 F$ & $g g F$ & $4 F$ & $5 F$ & $g g F$ & & & \\
\hline BP 1 & 55.64 & 206.50 & 4.00 & 33.05 & 115.00 & 1.96 & 267.96 & 4640.95 & $3.93(1.11)$ \\
\hline BP 2 & 34.41 & 158.05 & 2.03 & 18.67 & 82.06 & 0.71 & 196.74 & 2822.56 & $3.70(1.30)$ \\
\hline \multicolumn{10}{|c|}{ Using NLO $4 \mathrm{~F} b \bar{b} H$ process } \\
\hline BPs & \multicolumn{2}{|c|}{$4 F \mathrm{NLO}$} & $g g F$ & \multicolumn{2}{|c|}{$4 F \mathrm{NLO}$} & $g g F$ & $\begin{array}{c}\text { Total signal, } \\
\qquad S\end{array}$ & $\begin{array}{c}\text { Total background, } \\
\text { B }\end{array}$ & $\begin{array}{l}\frac{S}{\sqrt{B}} \text { without (with } \\
5 \% \text { ) systematics }\end{array}$ \\
\hline BP 1 & \multicolumn{2}{|c|}{133.01} & 4 & \multicolumn{2}{|c|}{66.94} & 1.96 & 205.91 & 4640.95 & $3.02(0.85)$ \\
\hline BP 2 & \multicolumn{2}{|c|}{89.27} & 2.03 & \multicolumn{2}{|c|}{46.0} & 0.71 & 138.01 & 2822.56 & $2.6(0.92)$ \\
\hline
\end{tabular}

Table 18. The signal yield along with signal significance for the b-tag $b \bar{b}+\mathbb{E}_{T}$ final state.

are $Z h, W h$ and $Z \gamma \gamma$. We generate the $Z h$ and $W h$ background upon merging with one additional jet in the final state where the SM Higgs decays to $\gamma \gamma$. For the $Z \gamma \gamma$, we decay the $Z$ boson into neutrinos and merge with one extra jet in the final state. Also, we generate $t \bar{t} h$ with $h \rightarrow \gamma \gamma$ which is a subdominant background to this final state. For details see appendix C.

4.2.2.1 b-veto category In this category, the selected event must contain exactly two photons with $p_{T}>30 \mathrm{GeV}$ and $|\eta|<2.5$ along with no b-jets satisfying $p_{T}>20 \mathrm{GeV}$ and $|\eta|<2.5$ in the final state. We veto events which contain leptons with $p_{T}>20 \mathrm{GeV}$ and $|\eta|<2.47$ (2.5) (for electron (muon)) in the final state to reduce the $p p \rightarrow \widetilde{\chi}_{1}^{ \pm} \widetilde{\chi}_{2,3}^{0}, Z h, W h$ and $t \bar{t} h$ backgrounds. Since photon has very clean signature with excellent mass resolution at the LHC, we restrict the di-photon invariant mass within $(122,128) \mathrm{GeV}$. The number of maximum light jets with $p_{T}>20 \mathrm{GeV}$ and $|\eta|<4.5$ are restricted to be one to reduce the backgrounds with multiple jets in the final state. Similar to the previous sections, we optimise the signal over backgrounds with missing energy, $\mathbb{E}_{T}$ and, the azimuthal angle separation between the di-photon system and $\mathbb{E}_{T}, \Delta \phi_{\gamma \gamma, \mathbb{E}_{T}}$. The normalised distribution of these variables for signal and dominant background events are shown in figure 20 .

The results of the optimised cuts for both of the benchmark points are displayed in table 19. The cut flow table for the benchmark point 1 is shown in table 20. We present the number of SM and susy background events at $\sqrt{s}=14 \mathrm{TeV}$ with $3 \mathrm{ab}^{-1}$ of integrated luminosity corresponding to each benchmark point in table 21. Also, we display the final signal significance in table 22 . The signal significance is very poor in this channel. However, the signal to background ratio, $S / B$ is large here which reduces the effect of adding systematic uncertainty on the final signal significance, viz. a $5 \%$ systematic changes the significance from 1.74 to 1.48 and 1.11 to 0.95 for the first benchmark point in case of matched $4 \mathrm{~F}, 5 \mathrm{~F} b \bar{b} H$ and NLO $4 F b \bar{b} H$ production respectively.

4.2.2.2 b-tag category Here, we select events with exactly two photon and atleast one extra b-jet in the final state meeting the basic cuts mentioned in the previous section 4.2.2.1. The event yield further reduces by demanding the extra b-jet. Next, we do a cut-based analysis with the kinematic variables, $\mathbb{E}_{T}$ and $\Delta \phi_{\gamma \gamma, \mathbb{E}_{T}}$ for the matched $4 F$ and $5 F b \bar{b} H$ 


\begin{tabular}{|c|c|}
\hline \multicolumn{2}{|c|}{ Selection cuts } \\
\hline BP 1 & \multicolumn{1}{|c|}{ BP 2} \\
\hline $2 \gamma, N_{\ell, b}=0$ \\
$122.0<m_{\gamma \gamma}<128.0$ \\
$N_{j} \leq 1$ \\
\hline $\mathscr{E}_{T}>150 \mathrm{GeV}$ & $\mathbb{E}_{T}>190 \mathrm{GeV}$ \\
$\Delta \phi_{\gamma \gamma, \mathbb{E}_{T}}>2.7$ & $\Delta \phi_{\gamma \gamma, \mathbb{E}_{T}}>2.5$ \\
\hline
\end{tabular}

Table 19. The selection cuts optimised in the $\gamma \gamma+\mathbb{E}_{T}$ channel for the cut-based analysis.
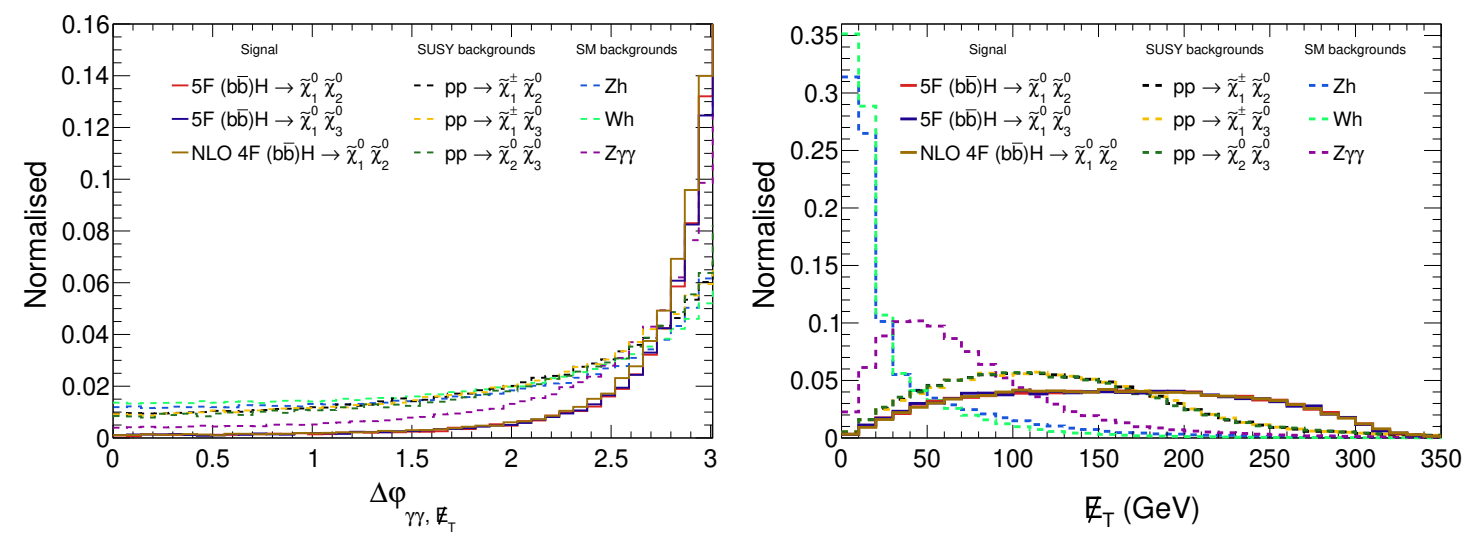

Figure 20. The normalised distributions of $\Delta \phi_{\gamma \gamma, \mathbb{E}_{T}}$ (left) and $\mathbb{E}_{T}$ (right) for the $\gamma \gamma+\mathbb{E}_{T}$ final state after the basic trigger cuts.

\begin{tabular}{|c|c|c|c|c|c|c|c|c|c|c|}
\hline \multirow{3}{*}{ Cut flow } & \multicolumn{10}{|c|}{ Event yield with $3 \mathrm{ab}^{-1}$ of integrated luminosity } \\
\hline & \multirow{2}{*}{$\begin{array}{c}\text { Signal } \\
b \bar{b} \rightarrow H(5 F)\end{array}$} & \multicolumn{5}{|c|}{ susy Backgrounds } & \multicolumn{4}{|c|}{ SM Backgrounds } \\
\hline & & $\widetilde{\chi}_{1}^{ \pm} \widetilde{\chi}_{2}^{0}$ & $\tilde{\chi}_{1}^{ \pm} \widetilde{\chi}_{3}^{0}$ & $\widetilde{\chi}_{2}^{0} \widetilde{\chi}_{3}^{0}$ & $\widetilde{\chi}_{1}^{0} \widetilde{\chi}_{2}^{0}$ & $\tilde{\chi}_{1}^{0} \widetilde{\chi}_{3}^{0}$ & $Z h$ & $W h$ & $Z \gamma \gamma$ & $t \bar{t} h$ \\
\hline $2 \gamma$ & 55.50 & 236.06 & 138.57 & 158.83 & 26.57 & 13.25 & 1782.53 & 2761.95 & 1663.45 & 175.10 \\
\hline$m_{\gamma \gamma}$ & 54.44 & 227.10 & 133.13 & 153.93 & 26.01 & 12.97 & 1751.64 & 2702.96 & 329.98 & 167.78 \\
\hline$N_{j}$ & 43.67 & 72.26 & 42.49 & 54.49 & 16.05 & 8.12 & 791.14 & 1122.31 & 259.10 & 2.53 \\
\hline $\mathbb{E}_{T}$ & 23.15 & 23.59 & 14.08 & 18.63 & 6.62 & 3.28 & 54.68 & 31.17 & 19.05 & 0.39 \\
\hline$\Delta \phi_{\gamma \gamma, \mathbb{E}_{T}}$ & 22.18 & 17.17 & 10.32 & 14.80 & 5.70 & 2.83 & 51.08 & 29.25 & 15.90 & 0.28 \\
\hline
\end{tabular}

Table 20. The cut-flow table for the benchmark point 1 in the $\gamma \gamma+\mathbb{E}_{T}$ mode with 5F signal production and backgrounds.

\begin{tabular}{|c|c|c|c|c|c|c|c|c|c|c|}
\hline \multirow[t]{3}{*}{ BPs } & \multicolumn{10}{|c|}{ Background yield at $3 \mathrm{ab}^{-1}$ after all cuts } \\
\hline & \multicolumn{5}{|c|}{ susy Backgrounds } & \multicolumn{4}{|c|}{ SM Backgrounds } & \multirow{3}{*}{$\begin{array}{c}\text { Total } \\
\text { Background, } \\
\text { B }\end{array}$} \\
\hline & $\tilde{\chi}_{1}^{ \pm} \widetilde{\chi}_{2}^{0}$ & $\tilde{\chi}_{1}^{ \pm} \widetilde{\chi}_{3}^{0}$ & $\widetilde{\chi}_{2}^{0} \widetilde{\chi}_{3}^{0}$ & $\widetilde{\chi}_{1}^{0} \widetilde{\chi}_{2}^{0}$ & $\tilde{\chi}_{1}^{0} \tilde{\chi}_{3}^{0}$ & $Z h$ & $W h$ & $Z \gamma \gamma$ & $t \bar{t} h$ & \\
\hline Order & \multicolumn{5}{|c|}{ NLO [154] } & \multicolumn{2}{|c|}{$\begin{array}{l}\text { NNLO (QCD)+ } \\
\text { NLO (EW) [161] }\end{array}$} & LO & NLO [161] & \\
\hline$\overline{B P} 1$ & 17.17 & 10.32 & 14.80 & 5.70 & 2.83 & (51.08 & 29.25 & 15.90 & 0.28 & 147.33 \\
\hline BP 2 & 10.90 & 6.23 & 9.35 & 5.22 & 2.57 & 30.02 & 14.82 & 7.83 & 0.15 & 87.09 \\
\hline
\end{tabular}

Table 21. The background yield at $14 \mathrm{TeV}$ with $3 \mathrm{ab}^{-1}$ of integrated luminosity after the cut-based analysis for the two benchmark points. 


\begin{tabular}{|c|c|c|c|c|c|c|c|c|c|}
\hline \multirow[t]{3}{*}{$\mathrm{BPs}$} & \multicolumn{6}{|c|}{ Signal rates at $3 \mathrm{ab}^{-1}$ after all cuts } & \multicolumn{3}{|c|}{ Significance calculation } \\
\hline & \multicolumn{3}{|c|}{$p p \rightarrow H / A \rightarrow \widetilde{\chi}_{1}^{0} \widetilde{\chi}_{2}^{0}$} & \multicolumn{3}{|c|}{$p p \rightarrow H / A \rightarrow \widetilde{\chi}_{1}^{0} \widetilde{\chi}_{3}^{0}$} & \multirow{2}{*}{$\begin{array}{c}\text { Total signal, } \\
\text { S }\end{array}$} & \multirow{2}{*}{$\begin{array}{c}\text { Total background, } \\
\text { B } \\
\text { (From table } 21)\end{array}$} & \multirow{2}{*}{$\begin{array}{c}\text { Significance, } \\
\frac{S}{\sqrt{B}} \text { without (with } \\
5 \% \text { ) systematics }\end{array}$} \\
\hline & $4 F$ & $5 F$ & $g g F$ & $4 F$ & $5 F$ & $g g F$ & & & \\
\hline BP 1 & 2.51 & 14.53 & 2.37 & 1.27 & 7.65 & 1.22 & 21.07 & 147.33 & $1.74(1.48)$ \\
\hline $\mathrm{BP} 2$ & 1.00 & 7.75 & 0.81 & 0.57 & 4.35 & 0.72 & 11.03 & 87.09 & $1.18(1.07)$ \\
\hline \multicolumn{10}{|c|}{ Using NLO $4 \mathrm{~F} b \bar{b} H$ process } \\
\hline $\mathrm{BPs}$ & \multicolumn{2}{|c|}{$4 F \mathrm{NLO}$} & $g g F$ & \multicolumn{2}{|c|}{$4 F \mathrm{NLO}$} & $g g F$ & $\begin{array}{c}\text { Total signal, } \\
S\end{array}$ & $\begin{array}{c}\text { Total background, } \\
\text { B }\end{array}$ & $\begin{array}{l}\frac{S}{\sqrt{B}} \text { without (with } \\
5 \% \text { ) systematics }\end{array}$ \\
\hline $\mathrm{BP} 1$ & \multicolumn{2}{|c|}{6.41} & 2.37 & \multicolumn{2}{|c|}{3.46} & 1.22 & 13.46 & 147.33 & $1.11(0.95)$ \\
\hline BP 2 & \multicolumn{2}{|c|}{3.34} & 0.81 & \multicolumn{2}{|c|}{1.77} & 0.72 & 6.64 & 87.09 & $0.71(0.64)$ \\
\hline
\end{tabular}

Table 22. The signal yield along with signal significance for the $\gamma \gamma+\mathbb{E}_{T}$ final state.

\begin{tabular}{|c|c|}
\hline \multicolumn{2}{|c|}{ Selection cuts } \\
\hline BP 1 & BP 2 \\
\hline \multicolumn{2}{|c|}{$\begin{array}{c}2 \gamma, N_{\ell}=0, N_{b} \geq 1 \\
122.0<m_{\gamma \gamma}<128.0 \\
N_{j} \leq 1\end{array}$} \\
\hline $\mathbb{E}_{T}>200 \mathrm{GeV}$ & $\mathbb{E}_{T}>230 \mathrm{GeV}$ \\
\hline$\Delta \phi_{\gamma \gamma, \mathbb{E}_{T}}>2.0$ & $\Delta \phi_{\gamma \gamma, \mathbb{E}_{T}}>2.1$ \\
\hline
\end{tabular}

Table 23. The selection cuts optimised in the b-tag $\gamma \gamma+\mathbb{E}_{T}$ channel for the cut-based analysis.

\begin{tabular}{|c|c|c|c|c|c|c|c|c|c|c|}
\hline \multirow[t]{3}{*}{ BPs } & \multicolumn{10}{|c|}{ Background yield at $3 \mathrm{ab}^{-1}$ after all cuts } \\
\hline & \multicolumn{5}{|c|}{ susy Backgrounds } & \multicolumn{4}{|c|}{ SM Backgrounds } & \multirow{2}{*}{$\begin{array}{c}\text { Total } \\
\text { Background, B }\end{array}$} \\
\hline & $\widetilde{\chi}_{1}^{ \pm} \widetilde{\chi}_{2}^{0}$ & $\widetilde{\chi}_{1}^{ \pm} \widetilde{\chi}_{3}^{0}$ & $\widetilde{\chi}_{2}^{0} \widetilde{\chi}_{3}^{0}$ & $\widetilde{\chi}_{1}^{0} \widetilde{\chi}_{2}^{0}$ & $\tilde{\chi}_{1}^{0} \widetilde{\chi}_{3}^{0}$ & $Z h$ & $W h$ & $Z \gamma \gamma$ & $t \bar{t} h$ & \\
\hline BP 1 & 0.63 & 0.37 & 2.29 & 0.13 & 0.06 & 0.33 & 0.33 & 0.24 & 1.36 & 5.74 \\
\hline BP 2 & 0.32 & 0.19 & 1.28 & 0.15 & 0.08 & 0.15 & 0.25 & 0.16 & 0.72 & 3.30 \\
\hline
\end{tabular}

Table 24. The background yield at $14 \mathrm{TeV}$ with $3 \mathrm{ab}^{-1}$ of integrated luminosity after the cut-based analysis for the two benchmark points in b-tag category.

process, and also with the signal process, NLO $4 F b \bar{b} H$. The selection cuts are presented in table 23 . We then present in table 25 the final signal significance calculated using the backgrounds given in table 24 .

Summary prompt final states. We end our discussion of Higgs to susy decays in prompt final state with a summary. We evaluate the prospect of discovering heavy Higgs in electroweakino decays at the HL-LHC. The signature for such decays are mono- $Z+\mathbb{E}_{T}$ and mono- $h+\mathbb{E}_{T}$ final state. The leptonic decay of $Z$ boson in mono- $Z+\mathbb{E}_{T}$ final state gives rise to $\ell \ell+\mathbb{E}_{T}$ channel. In case of mono- $h+\mathbb{E}_{T}$ final state, we consider the decay of the SM Higgs into $b b$ and $\gamma \gamma$ final state which gives rise to $b \bar{b}+\mathbb{E}_{T}$ and $\gamma \gamma+\mathbb{E}_{T}$ channel respectively. Further, we divide our analysis into b-veto and b-tag category to account for extra b-jet requirement in case of bottom pair fusion production mode of heavy Higgs. 


\begin{tabular}{|c|c|c|c|c|c|c|c|c|c|}
\hline \multirow[t]{3}{*}{ BPs } & \multicolumn{6}{|c|}{ Signal rates at $3 \mathrm{ab}^{-1}$ after all cuts } & \multicolumn{3}{|c|}{ Significance calculation } \\
\hline & \multicolumn{3}{|c|}{$p p \rightarrow H / A \rightarrow \widetilde{\chi}_{1}^{0} \widetilde{\chi}_{2}^{0}$} & \multicolumn{3}{|c|}{$p p \rightarrow H / A \rightarrow \widetilde{\chi}_{1}^{0} \widetilde{\chi}_{3}^{0}$} & \multirow{2}{*}{$\begin{array}{c}\text { Total signal, } \\
\text { S }\end{array}$} & \multirow{2}{*}{$\begin{array}{c}\text { Total background, } \\
\text { B } \\
\text { (From table } 24)\end{array}$} & \multirow{2}{*}{$\begin{array}{c}\text { Significance, } \\
\frac{S}{\sqrt{B}} \text { without (with } \\
5 \% \text { ) systematics }\end{array}$} \\
\hline & $4 F$ & $5 F$ & $g g F$ & $4 F$ & $5 F$ & $g g F$ & & & \\
\hline BP 1 & 1.11 & 3.86 & 0.068 & 0.58 & 2.10 & 0.034 & 4.97 & 5.74 & $2.07(2.07)$ \\
\hline BP 2 & 0.54 & 2.47 & 0.023 & 0.31 & 1.30 & 0.02 & 3.09 & 3.30 & $1.70(1.70)$ \\
\hline \multicolumn{10}{|c|}{ Using NLO $4 \mathrm{~F} b \bar{b} H$ process } \\
\hline BPs & \multicolumn{2}{|c|}{$4 F \mathrm{NLO}$} & $g g F$ & \multicolumn{2}{|c|}{$4 F \mathrm{NLO}$} & $g g F$ & $\begin{array}{c}\text { Total signal, } \\
S\end{array}$ & $\begin{array}{c}\text { Total background, } \\
\text { B }\end{array}$ & $\begin{array}{l}\frac{S}{\sqrt{B}} \text { without (with } \\
5 \% \text { ) systematics }\end{array}$ \\
\hline BP 1 & \multicolumn{2}{|c|}{2.52} & 0.068 & \multicolumn{2}{|c|}{1.33} & 0.034 & 3.95 & 5.74 & $1.65(1.64)$ \\
\hline BP 2 & \multicolumn{2}{|c|}{1.51} & 0.023 & \multicolumn{2}{|c|}{0.81} & 0.02 & 2.36 & 3.30 & $1.3(1.29)$ \\
\hline
\end{tabular}

Table 25. The signal yield along with signal significance for the b-tag $\gamma \gamma+\mathbb{E}_{T}$ final state.

The $\ell \ell+\mathbb{E}_{T}$ channel can give rise to promising signature at the collider with higher signal significance. The result improves up to $\sim 70 \%$ upon considering b-tag category. For the case of $b \bar{b}+\mathbb{E}_{T}$ channel the signal to background ratio is very poor, mainly because of large $Z b \bar{b}$ and $t \bar{t}$ background. To probe heavy Higgs in this channel one has to look for better ways to reduce these backgrounds. The $\gamma \gamma+\mathbb{E}_{T}$ channel suffers from smaller event rate in spite of being a clean final state. This channel might be very important search channel at higher energy colliders like HE-LHC or $100 \mathrm{TeV}$ collider. We would like to stress that our work for the first time, demonstrates the importance and the impact of susy backgrounds for Higgs to susy decays. The production cross section of susy backgrounds can be comparable or larger than the signal processes considered here. This depends on exact details of parameter space, most importantly, the composition of electroweakinos. The relative importance of signal and susy background process for few benchmark scenarios can be seen in table 3 . Apart from an overall increase in total background cross sections due to susy processes, we observe an increased overlap in the kinematic distributions e.g. $\Delta R_{b b}$ and $\mathbb{E}_{T}$ as shown figure 17. These features of susy backgrounds altogether can lead to an appreciable amount of contribution in the total background. Hence one must appropriately take into account these susy backgrounds into the analysis while searching for heavy Higgs decays in mono-X signatures at the collider.

\section{$5 \quad$ Long lived charged particle (LLCP)}

Along with the prompt final states discussed in last sections, it is also possible that heavy Higgs decays into long lived charginos (with path lengths of few centimetres in the detector). Such long lived chargino can then decay with a soft final state in the detector, leaving the so called disappearing track. In this section, we turn our focus on these scenarios. Recall that the kinematic feature of these type of final states were discussed in section 3.3. As we have discussed earlier, for a winolike LSP the mass gap between $\widetilde{\chi}_{1}^{0}$ and $\widetilde{\chi}_{1}^{ \pm}$is very small and the $\tilde{\chi}_{1}^{ \pm}$becomes long lived charged particle (LLCP). We discuss the features of these LLCP and prospects of observing them at collider in the following sections. It should be noted that the heavy neutral Higgs will decay to pair of charginos. In case heavy Higgs 


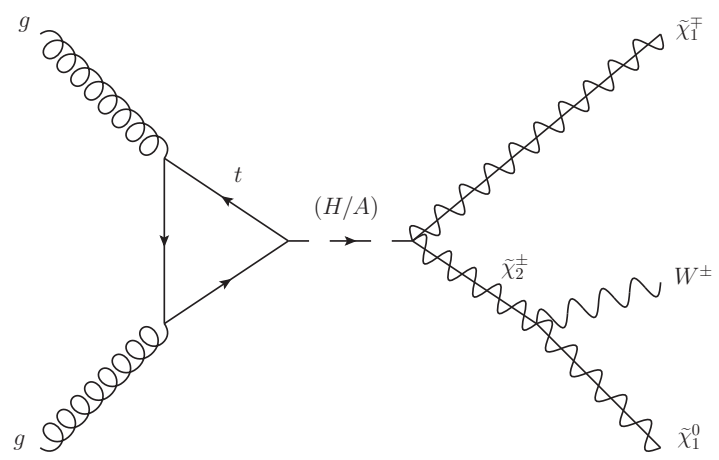

Figure 21. The Feynman diagram for the production of $\widetilde{\chi}_{1}^{\mp}$ from $p p \rightarrow H \rightarrow \widetilde{\chi}_{1}^{\mp} \widetilde{\chi}_{2}^{ \pm}, \widetilde{\chi}_{2}^{ \pm} \rightarrow$ $W^{ \pm} \widetilde{\chi}_{1}^{0}$ process.

decays to pair of light chargino, the existing disappearing track searches are applicable. However as shown previously in section 3.1, heavy Higgs can have a significant branching ratio to $\widetilde{\chi}_{1}^{ \pm} \widetilde{\chi}_{2}^{ \pm}$. The $\widetilde{\chi}_{2}^{ \pm}$decays promptly with visible final states (e.g. leptons, photons or jets). While the existing disappearing track searches $[163,164]$ are sensitive to presence of additional jets in the final state, they veto energetic leptons. In addition to LLCP production via neutral Higgs, we also review charged Higgs signature which can give rise to disappearing track. This search channel also suffers from same problem of generating additional hits inside tracker which are usually vetoed out in the existing search strategies.

Before we proceed, a comment about choice of benchmarks in this section is in order. In section 2, we discussed the impact of loop corrections on the predicted chargino decay length. In this work, we have not taken into account such loop corrections. However, the results being discussed depend on the mass hierarchy between the heavy Higgs and the chargino. Including the loop corrections will largely impact the chargino decay length while the mass hierarchy will largely be unaltered. Therefore, we consider that the choice of our benchmark points is justified irrespective of the missing loop corrections to chargino decay lengths.

\subsection{Decay fraction of LLCP at various tracker ranges in the detector}

Here, we take three benchmark points (BPs) with a LLCP $\widetilde{\chi}_{1}^{ \pm}$, which satisfy all the collider constraints as discussed in section 3. It should be noted that SModels database currently does not include disappearing track analyses results. These results may pose additional constraints on the parameter space which are not taken into account here. For illustrative purposes, we consider the $\tilde{\chi}_{1}^{ \pm}$decay lengths of $3 \mathrm{~mm}, 3 \mathrm{~cm}$ and $30 \mathrm{~cm}$ corresponding to the benchmarks in equation (5.1), (5.2) and (5.3) respectively. The LLCP is produced via the process, $p p \rightarrow H \rightarrow \tilde{\chi}_{1}^{\mp} \tilde{\chi}_{2}^{ \pm}, \widetilde{\chi}_{2}^{ \pm} \rightarrow W^{ \pm} \tilde{\chi}_{1}^{0}$ (figure 21). We trigger these events by applying cuts on the transverse momentum, $p_{T}$ and pseudorapidity, $\eta$, of $\mathrm{W}$ boson decay products. The analysis is divided into three parts depending on the trigger. The first one is on the lepton from the decay of $\mathrm{W}$ boson which must satisfy $p_{T, \ell}>30 \mathrm{GeV},\left|\eta_{\ell}\right|<2.5$. For the second trigger, the events must contain at least one jet with $p_{T, j}>200 \mathrm{GeV}$ and $\left|\eta_{j}\right|<2.5$. The events with at least two jets with $p_{T, j}>150 \mathrm{GeV}$ and $\left|\eta_{j}\right|<2.5$ are selected in the 


\begin{tabular}{|c|c|}
\hline Trigger & Cuts \\
\hline Trigger 1 & $p_{T, \ell}>30 \mathrm{GeV},\left|\eta_{\ell}\right|<2.5$ \\
\hline Trigger 2 & At least one jet with $p_{T}>200 \mathrm{GeV}$ and $|\eta|<2.5$ \\
\hline Trigger 3 & At least two jets with $p_{T}>150 \mathrm{GeV}$ and $|\eta|<2.5$ \\
\hline
\end{tabular}

Table 26. Summarizing trigger cuts for LLCP scenario. Along with these trigger cuts, additional constraints are applied on $\widetilde{\chi}_{1}^{ \pm}$which are $p_{T, \widetilde{\chi}_{1}^{ \pm}}>100 \mathrm{GeV}$ and $\left|\eta_{\widetilde{\chi}_{1}^{ \pm}}\right|<2.5$.

third trigger. We also demand that the $\widetilde{\chi}_{1}^{ \pm}$must be produced with $p_{T, \tilde{\chi}_{1}^{ \pm}}>100 \mathrm{GeV}$ within $|\eta|<2.5$ in all the trigger choices. These choices are summarised in table 26. The whole set-up and the analysis is done in the Pythia-6 framework. We expect use of Pythia8 for event generation to produce largely identical results because the kinematic distribution of observables are identical in Pythia-6 and Pythia-8 which makes the final result unaltered. The event yield of this process after applying these trigger cuts along with the production cross section and the decay branching ratios for different benchmark points are listed in table 27 at the HL-LHC with $3 \mathrm{ab}^{-1}$ of integrated luminosity. We also show the normalised distributions of the mean decay length, $\beta c \gamma \tau$, obtained after applying trigger cuts, in case of the three chosen BP's corresponding to different decay lengths in figure 22 .

$$
\begin{aligned}
& M_{A}=1.8 \mathrm{TeV}, \tan \beta=16.3, M_{1}=387 \mathrm{GeV}, M_{2}=124 \mathrm{GeV}, \mu=303 \mathrm{GeV}, \\
& M_{3}=4.7 \mathrm{TeV}, M_{\tilde{Q}_{1_{L}}, \tilde{Q}_{2_{L}}}=M_{\tilde{u}_{R}, \tilde{d}_{R}, \tilde{c}_{R}, \tilde{s}_{R}}=M_{\tilde{e}_{L}, \tilde{\mu}_{L}, \tilde{e}_{R}, \tilde{\mu}_{R}}=3 \mathrm{TeV}, M_{\tilde{Q}_{3_{L}}}=7.8 \mathrm{TeV}, \\
& A_{t}=-2.4 \mathrm{TeV}, A_{b}=0.5 \mathrm{TeV}, A_{\tau}=-1 \mathrm{TeV}, A_{e, \mu, u, d, c, s}=0, \\
& M_{\tilde{\tau}_{L}}=1.8 \mathrm{TeV}, M_{\tilde{\tau}_{R}}=2 \mathrm{TeV}, M_{\tilde{t}_{R}}=3.7 \mathrm{TeV}, M_{\tilde{b}_{R}}=2.9 \mathrm{TeV} \\
& M_{A}=1.6 \mathrm{TeV}, \tan \beta=43.7, M_{1}=913 \mathrm{GeV}, M_{2}=154 \mathrm{GeV}, \mu=347 \mathrm{GeV}, \\
& M_{3}=2.4 \mathrm{TeV}, M_{\tilde{Q}_{1_{L}}, \tilde{Q}_{2_{L}}}=M_{\tilde{u}_{R}, \tilde{d}_{R}, \tilde{c}_{R}, \tilde{s}_{R}}=M_{\tilde{e}_{L}, \tilde{\mu}_{L}, \tilde{e}_{R}, \tilde{\mu}_{R}}=3 \mathrm{TeV}, M_{\tilde{Q}_{3_{L}}}=7.3 \mathrm{TeV}, \\
& A_{t}=-6.2 \mathrm{TeV}, A_{b}=-86 \mathrm{GeV}, A_{\tau}=-1.6 \mathrm{TeV}, A_{e, \mu, u, d, c, s}=0, \\
& M_{\tilde{\tau}_{L}}=1 \mathrm{TeV}, M_{\tilde{\tau}_{R}}=1.9 \mathrm{TeV}, M_{\tilde{t}_{R}}=6.4 \mathrm{TeV}, M_{\tilde{b}_{R}}=8.2 \mathrm{TeV} \\
& M_{A}=1.5 \mathrm{TeV}, \tan \beta=5.8, M_{1}=618 \mathrm{GeV}, M_{2}=308 \mathrm{GeV}, \mu=627 \mathrm{GeV}, \\
& M_{3}=4.9 \mathrm{TeV}, M_{\tilde{Q}_{1_{L}}, \tilde{Q}_{2_{L}}}=M_{\tilde{u}_{R}, \tilde{d}_{R}, \tilde{c}_{R}, \tilde{s}_{R}}=M_{\tilde{e}_{L}, \tilde{\mu}_{L}, \tilde{e}_{R}, \tilde{\mu}_{R}}=3 \mathrm{TeV}, M_{\tilde{Q}_{3_{L}}}=6.2 \mathrm{TeV}, \\
& A_{t}=4.4 \mathrm{TeV}, A_{b}=-264 \mathrm{GeV}, A_{\tau}=1.9 \mathrm{TeV}, A_{e, \mu, u, d, c, s}=0, \\
& M_{\tilde{\tau}_{L}}=0.8 \mathrm{TeV}, M_{\tilde{\tau}_{R}}=1.1 \mathrm{TeV}, M_{\tilde{t}_{R}}=4.2 \mathrm{TeV}, M_{\tilde{b}_{R}}=0.8 \mathrm{TeV}
\end{aligned}
$$

We compute the fractional number of events where chargino decays within various distances inside detector as the ratio of number of events within that range divided by the number of events passing trigger criterion. We quote these numbers in table 28 for different $\tilde{\chi}_{1}^{ \pm}$decay lengths. From table 28, it is evident that due to the Lorentz factors, the $\tilde{\chi}_{1}^{ \pm}$decays mostly at larger distances with respect to its decay length. Such highly boosted chargino can improve the sensitivity of the disappearing track searches as they live for longer time in the detector. The existing disappearing track searches fail below a chargino decay length below approximately $3 \mathrm{~mm}$, however the boosted chargino produced via heavy Higgs with such low decay length can lead to an additional handle for such scenarios. 

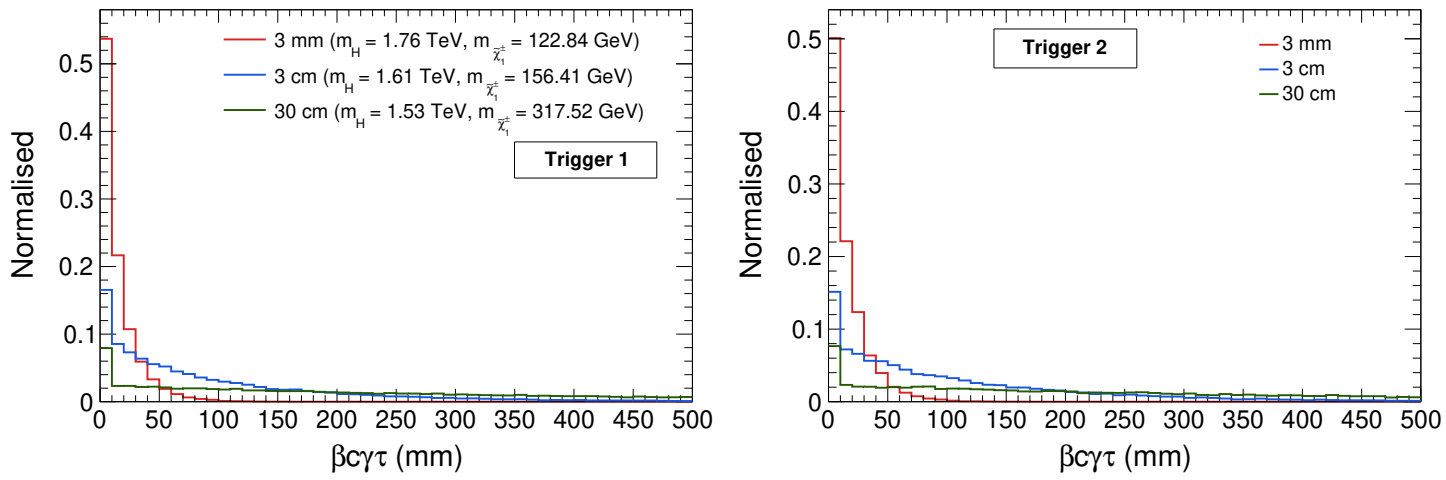

Figure 22. The normalised distribution of mean decay length for the three LLCP scenario after requiring Trigger 1 (left) and Trigger 2 (right) along with additional selection criteria.

\begin{tabular}{|c|c|c|c|c|c|}
\hline \multirow{2}{*}{$\begin{array}{c}\beta c \gamma \tau \text { of } \\
\tilde{\chi}_{1}^{ \pm}\end{array}$} & \multirow{2}{*}{$\begin{array}{c}\text { Cross-section of } \\
g g / b \bar{b} \rightarrow H / A(\mathrm{fb})\end{array}$} & \multirow{2}{*}{$\begin{array}{c}\text { Branching ratio } \\
(\%)\end{array}$} & \multirow[t]{2}{*}{ Trigger cuts } & \multicolumn{2}{|c|}{ Event yield at $3 a b^{-1}$} \\
\hline & & & & before trigger & after trigger \\
\hline \multirow{3}{*}{$3 \mathrm{~mm}$} & \multirow{3}{*}{1.51} & \multirow{3}{*}{$\begin{array}{c}B R\left(H \rightarrow \widetilde{\chi}_{1}^{ \pm} \widetilde{\chi}_{2}^{\mp}\right)=40.23 \\
B R\left(A \rightarrow \widetilde{\chi}_{1}^{ \pm} \widetilde{\chi}_{2}^{\mp}\right)=38.33 \\
B R\left(\widetilde{\chi}_{2}^{ \pm} \rightarrow W^{ \pm} \widetilde{\chi}_{1}^{0}\right)=37.75\end{array}$} & Trigger 1 & 437.56 & 314.52 \\
\hline & & & Trigger 2 & \multirow{2}{*}{905.87} & 403.73 \\
\hline & & & Trigger 3 & & 125.30 \\
\hline \multirow{3}{*}{$3 \mathrm{~cm}$} & \multirow{3}{*}{18.97} & \multirow{3}{*}{$\begin{array}{c}B R\left(H \rightarrow \widetilde{\chi}_{1}^{ \pm} \widetilde{\chi}_{2}^{ \pm}\right)=16.82 \\
B R\left(A \rightarrow \widetilde{\chi}_{1}^{ \pm} \widetilde{\chi}_{2}^{ \pm}\right)=16.42 \\
B R\left(\widetilde{\chi}_{2}^{ \pm} \rightarrow W^{ \pm} \widetilde{\chi}_{1}^{0}\right)=37.13\end{array}$} & Trigger 1 & 2287.66 & 1617.88 \\
\hline & & & Trigger 2 & \multirow{2}{*}{4736.18} & 1796.15 \\
\hline & & & Trigger 3 & & 460.36 \\
\hline \multirow{3}{*}{$30 \mathrm{~cm}$} & \multirow{3}{*}{0.93} & \multirow{3}{*}{$\begin{array}{c}B R\left(H \rightarrow \widetilde{\chi}_{1}^{ \pm} \widetilde{\chi}_{2}^{ \pm}\right)=48.77 \\
B R\left(A \rightarrow \widetilde{\chi}_{1}^{ \pm} \widetilde{\chi}_{2}^{ \pm}\right)=45.07 \\
B R\left(\widetilde{\chi}_{2}^{ \pm} \rightarrow W^{ \pm} \widetilde{\chi}_{1}^{0}\right)=33.72\end{array}$} & Trigger 1 & 287.54 & 221.41 \\
\hline & & & Trigger 2 & \multirow{2}{*}{595.30} & 241.04 \\
\hline & & & Trigger 3 & & 49.49 \\
\hline
\end{tabular}

Table 27. Production cross-section and branching ratios for all the three benchmark points along with the yield after putting trigger cuts at $3 \mathrm{ab}^{-1}$. For the leptonic case, the $\mathrm{W}$ boson from $\tilde{\chi}_{2}^{ \pm}$is decayed leptonically $(\ell=e, \mu, \tau)$, and the $\mathrm{W}$ boson decays to jets for the case of jet trigger.

\begin{tabular}{|c||c|c|c|c|c|c|c|}
\hline Trigger cuts & $\beta c \gamma \tau$ of & \multicolumn{6}{|c|}{ Fraction of events after trigger in \% within } \\
\cline { 2 - 8 } & $\tilde{\chi}_{1}^{ \pm}$ & $0-3 \mathrm{~mm}$ & $3-30 \mathrm{~mm}$ & $30 \mathrm{~mm}-10 \mathrm{~cm}$ & $10-30 \mathrm{~cm}$ & $30-100 \mathrm{~cm}$ & $>100 \mathrm{~cm}$ \\
\hline \multirow{3}{*}{ Trigger 1} & $3 \mathrm{~mm}$ & 26.61 & 59.49 & 13.58 & 0.32 & 0.0 & 0.0 \\
\cline { 2 - 8 } & $3 \mathrm{~cm}$ & 9.83 & 22.56 & 32.53 & 28.48 & 6.57 & 0.03 \\
\cline { 2 - 8 } & $30 \mathrm{~cm}$ & 6.16 & 6.27 & 14.07 & 27.99 & 35.27 & 10.24 \\
\hline \hline \multirow{3}{*}{ Trigger 2} & $3 \mathrm{~mm}$ & 24.07 & 60.49 & 15.10 & 0.34 & 0.0 & 0.0 \\
\cline { 2 - 8 } & $3 \mathrm{~cm}$ & 9.49 & 19.44 & 31.62 & 31.89 & 7.51 & 0.05 \\
\cline { 2 - 8 } & $30 \mathrm{~cm}$ & 5.86 & 6.01 & 13.64 & 27.66 & 36.39 & 10.44 \\
\hline \hline \multirow{3}{*}{ Trigger 3 } & $3 \mathrm{~mm}$ & 23.06 & 60.75 & 15.86 & 0.33 & 0.0 & 0.0 \\
\cline { 2 - 8 } & $3 \mathrm{~cm}$ & 9.03 & 18.01 & 31.58 & 32.79 & 8.53 & 0.06 \\
\cline { 2 - 8 } & $30 \mathrm{~cm}$ & 5.90 & 5.63 & 13.04 & 27.07 & 37.53 & 10.83 \\
\hline
\end{tabular}

Table 28. After triggering event with $p_{T}$ and $\eta$ cut, the fraction of charginos which decay in different regions of tracker (or outside) in detector with decay length of a few mm upto few centimeters. 


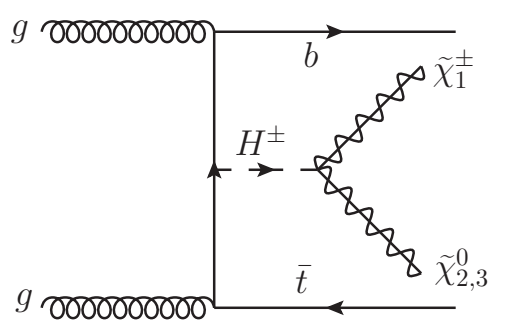

(a)

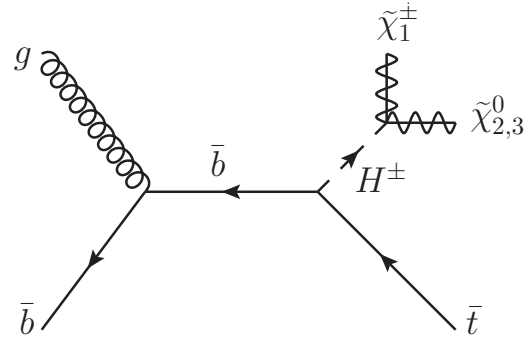

(b)

Figure 23. The Feynman diagram of (a) $4 F$ and (b) $5 F$ charged Higgs production at LO, which decays to electroweakinos yielding LLCP signature at the collider.

\subsection{Probing charged Higgs via LLCP signature}

Finally, we introduce another probe of new physics, namely supersymmetric decays of charged Higgs. The supersummetric final states arising from charged Higgs decays have so far not been analysed in the literature. A complete overview of these decays is beyond the scope of this work. However, we illustrate an example of charged Higgs decays to LLCP. At the LHC, the $H^{ \pm}$is already being searched for by its decay into various Standard Model (SM) particles, viz. $H^{ \pm} \rightarrow \tau^{ \pm} \nu[165-168]$ and $H^{ \pm} \rightarrow t \bar{b}[169,170]$. Below top quark mass, it is mainly produced from the top quark decay in $t \bar{t}$ production, $t \rightarrow H^{+} b$. For the case of $m_{H^{ \pm}}>m_{t}$, the charged Higgs production happens via two processes, viz. four-flavour $(4 F), g g / q \bar{q} \rightarrow t \bar{b} H^{-}$and five-flavour $(5 F), g b \rightarrow t H^{-}$. Then, these two processes are matched to get the total inclusive cross-section, in the same way as we have discussed at the beginning of section 4 .

In the MSSM, the charged Higgs can decay into a pair of electroweakinos, viz. $H^{ \pm} \rightarrow$ $\tilde{\chi}_{1}^{ \pm} \widetilde{\chi}_{2}^{0}$ (figure 23). For a winolike LSP scenario, the $\widetilde{\chi}_{1}^{ \pm}$produced from the decay of $H^{ \pm}$ can be long-lived. Therefore, the charged Higgs can be probed via missing charged track signature. A demonstration of possible analysis is our main goal in this section. For this, we choose the following three benchmark points from our scan, equation (5.4), (5.5) and (5.6) which corresponds to charged Higgs mass around 600,800 and $1000 \mathrm{GeV}$ respectively. The $4 F$ and $5 F$ production cross-section for charged Higgs and various electroweakino branching ratios for these benchmark points are given in table 29 .

$$
\begin{aligned}
& M_{A}=615 \mathrm{GeV}, \tan \beta=13.1, M_{1}=485 \mathrm{GeV}, M_{2}=138 \mathrm{GeV}, \mu=370 \mathrm{GeV}, \\
& M_{3}=4.1 \mathrm{TeV}, M_{\tilde{Q}_{1_{L}}, \tilde{Q}_{2_{L}}}=M_{\tilde{u}_{R}, \tilde{d}_{R}, \tilde{c}_{R}, \tilde{s}_{R}}=M_{\tilde{e}_{L}, \tilde{\mu}_{L}, \tilde{e}_{R}, \tilde{\mu}_{R}}=3 \mathrm{TeV}, M_{\tilde{Q}_{3_{L}}}=7.5 \mathrm{TeV}, \\
& A_{t}=4.5 \mathrm{TeV}, A_{b}=-1.3 \mathrm{TeV}, A_{\tau}=-1.6 \mathrm{TeV}, A_{e, \mu, u, d, c, s}=0, \\
& M_{\tilde{\tau}_{L}}=297 \mathrm{GeV}, M_{\tilde{\tau}_{R}}=1.4 \mathrm{TeV}, M_{\tilde{t}_{R}}=3.5 \mathrm{TeV}, M_{\tilde{b}_{R}}=4.4 \mathrm{TeV} \\
& M_{A}=815 \mathrm{GeV}, \tan \beta=17.5, M_{1}=734 \mathrm{GeV}, M_{2}=105 \mathrm{GeV}, \mu=335 \mathrm{GeV}, \\
& M_{3}=4 \mathrm{TeV}, M_{\tilde{Q}_{1_{L}}, \tilde{Q}_{2_{L}}}=M_{\tilde{u}_{R}, \tilde{d}_{R}, \tilde{c}_{R}, \tilde{s}_{R}}=M_{\tilde{e}_{L}, \tilde{\mu}_{L}, \tilde{e}_{R}, \tilde{\mu}_{R}}=3 \mathrm{TeV}, M_{\tilde{Q}_{3_{L}}}=7.1 \mathrm{TeV}, \\
& A_{t}=695 \mathrm{GeV}, A_{b}=1 \mathrm{TeV}, A_{\tau}=0.2 \mathrm{TeV}, A_{e, \mu, u, d, c, s}=0, \\
& M_{\tilde{\tau}_{L}}=1 \mathrm{TeV}, M_{\tilde{\tau}_{R}}=1.8 \mathrm{TeV}, M_{\tilde{t}_{R}}=4.8 \mathrm{TeV}, M_{\tilde{b}_{R}}=4.3 \mathrm{TeV}
\end{aligned}
$$




\begin{tabular}{|c|c|c|c|c|}
\hline \multirow{2}{*}{$\begin{array}{l}m_{H^{ \pm}} \\
(\mathrm{GeV})\end{array}$} & \multicolumn{3}{|c|}{ Cross-section at NLO (fb) } & \multirow{2}{*}{$\begin{array}{c}\text { Branching ratio } \\
(\%)\end{array}$} \\
\hline & $(4 F)$ & $(5 F)$ & Matched & \\
\hline $\begin{array}{c}620.29 \\
\text { eq. }(5.4)\end{array}$ & 11.92 & 16.72 & 15.48 & $\begin{array}{c}B R\left(H^{ \pm} \rightarrow \widetilde{\chi}_{1}^{ \pm} \widetilde{\chi}_{2}^{0}\right)=21.70, B R\left(H^{ \pm} \rightarrow \widetilde{\chi}_{1}^{ \pm} \widetilde{\chi}_{3}^{0}\right)=19.76 \\
B R\left(H^{ \pm} \rightarrow \widetilde{\chi}_{2}^{ \pm} \widetilde{\chi}_{1}^{0}\right)=19.93, B R\left(\widetilde{\chi}_{2}^{0} \rightarrow Z \widetilde{\chi}_{1}^{0}\right)=5.55 \\
B R\left(\widetilde{\chi}_{2}^{0} \rightarrow h \widetilde{\chi}_{1}^{0}\right)=23.16, B R\left(\widetilde{\chi}_{3}^{0} \rightarrow Z \widetilde{\chi}_{1}^{0}\right)=27.88 \\
B R\left(\widetilde{\chi}_{3}^{0} \rightarrow h \widetilde{\chi}_{1}^{0}\right)=3.80, B R\left(\widetilde{\chi}_{2}^{ \pm} \rightarrow Z \widetilde{\chi}_{1}^{ \pm}\right)=34.89 \\
B R\left(\widetilde{\chi}_{2}^{ \pm} \rightarrow h \widetilde{\chi}_{1}^{ \pm}\right)=27.79\end{array}$ \\
\hline $\begin{array}{c}819.77 \\
\text { eq. }(5.5)\end{array}$ & 6.48 & 9.33 & 8.64 & $\begin{array}{c}B R\left(H^{ \pm} \rightarrow \widetilde{\chi}_{1}^{ \pm} \widetilde{\chi}_{2}^{0}\right)=20.49, B R\left(H^{ \pm} \rightarrow \widetilde{\chi}_{1}^{ \pm} \widetilde{\chi}_{3}^{0}\right)=21.79 \\
B R\left(H^{ \pm} \rightarrow \widetilde{\chi}_{2}^{ \pm} \widetilde{\chi}_{1}^{0}\right)=22.52, B R\left(\widetilde{\chi}_{2}^{0} \rightarrow Z \widetilde{\chi}_{1}^{0}\right)=26.68 \\
B R\left(\widetilde{\chi}_{2}^{0} \rightarrow h \widetilde{\chi}_{1}^{0}\right)=4.54, B R\left(\widetilde{\chi}_{3}^{0} \rightarrow Z \widetilde{\chi}_{1}^{0}\right)=7.00 \\
B R\left(\widetilde{\chi}_{3}^{0} \rightarrow h \widetilde{\chi}_{1}^{0}\right)=21.81, B R\left(\widetilde{\chi}_{2}^{ \pm} \rightarrow Z \widetilde{\chi}_{1}^{ \pm}\right)=35.74 \\
B R\left(\widetilde{\chi}_{2}^{ \pm} \rightarrow h \widetilde{\chi}_{1}^{ \pm}\right)=27.74\end{array}$ \\
\hline $\begin{array}{l}1077.18 \\
\text { eq. }(5.6)\end{array}$ & 2.46 & 3.77 & 3.47 & $\begin{array}{c}B R\left(H^{ \pm} \rightarrow \widetilde{\chi}_{1}^{ \pm} \widetilde{\chi}_{2}^{0}\right)=19.24, B R\left(H^{ \pm} \rightarrow \widetilde{\chi}_{1}^{ \pm} \widetilde{\chi}_{3}^{0}\right)=20.52 \\
B R\left(H^{ \pm} \rightarrow \widetilde{\chi}_{2}^{ \pm} \widetilde{\chi}_{1}^{0}\right)=22.23, B R\left(\widetilde{\chi}_{2}^{0} \rightarrow Z \widetilde{\chi}_{1}^{0}\right)=26.72 \\
B R\left(\widetilde{\chi}_{2}^{0} \rightarrow h \widetilde{\chi}_{1}^{0}\right)=3.55, B R\left(\widetilde{\chi}_{3}^{0} \rightarrow Z \widetilde{\chi}_{1}^{0}\right)=6.59 \\
B R\left(\widetilde{\chi}_{3}^{0} \rightarrow h \widetilde{\chi}_{1}^{0}\right)=20.62, B R\left(\widetilde{\chi}_{2}^{ \pm} \rightarrow Z \widetilde{\chi}_{1}^{ \pm}\right)=36.17 \\
B R\left(\widetilde{\chi}_{2}^{ \pm} \rightarrow h \widetilde{\chi}_{1}^{ \pm}\right)=26.04\end{array}$ \\
\hline
\end{tabular}

Table 29. The production cross-section and branching ratios for the selected benchmark points in charged Higgs analysis.

$$
\begin{aligned}
& M_{A}=1.1 \mathrm{TeV}, \tan \beta=21.4, M_{1}=982 \mathrm{GeV}, M_{2}=119 \mathrm{GeV}, \mu=311 \mathrm{GeV}, \\
& M_{3}=4.4 \mathrm{TeV}, M_{\tilde{Q}_{1}, \tilde{Q}_{2_{L}}}=M_{\tilde{u}_{R}, \tilde{d}_{R}, \tilde{c}_{R}, \tilde{s}_{R}}=M_{\tilde{e}_{L}, \tilde{\mu}_{L}, \tilde{e}_{R}, \tilde{\mu}_{R}}=3 \mathrm{TeV}, M_{\tilde{Q}_{3_{L}}}=5.8 \mathrm{TeV}, \\
& A_{t}=5.3 \mathrm{TeV}, A_{b}=92 \mathrm{GeV}, A_{\tau}=-985 \mathrm{GeV}, A_{e, \mu, u, d, c, s}=0, \\
& M_{\tilde{\tau}_{L}}=1.3 \mathrm{TeV}, M_{\tilde{\tau}_{R}}=1 \mathrm{TeV}, M_{\tilde{t}_{R}}=2.2 \mathrm{TeV}, M_{\tilde{b}_{R}}=1.8 \mathrm{TeV}
\end{aligned}
$$

Again, we use Pythia- 6 to generate charged Higgs production in both the $4 F$ and $5 F$ scheme, whereas we compute the cross-sections at NLO using MadGraph-2.6.5 with the model file [171] made by the authors of [172]. While generating the cross-sections, we set the factorisation and renormalisation scales at $\mu=\left(m_{H^{ \pm}}+m_{t}\right) / 2$. This cross-section depends strongly on the scale variation of the bottom mass which has not been included in the above model file. We take care of it by rescaling the cross-section according to the running of bottom quark mass given in [173].

\subsection{1 $\ell \ell+$ LLCP}

Here, we consider the following decay processes of the $H^{ \pm}$, viz.

$$
\begin{array}{ll}
H^{ \pm} \rightarrow \widetilde{\chi}_{1}^{ \pm} \tilde{\chi}_{2,3}^{0}, & \tilde{\chi}_{2,3}^{0} \rightarrow \widetilde{\chi}_{1}^{0}+(Z \rightarrow \ell \ell), \\
H^{ \pm} \rightarrow \widetilde{\chi}_{2}^{ \pm} \tilde{\chi}_{1}^{0}, & \tilde{\chi}_{2}^{ \pm} \rightarrow \widetilde{\chi}_{1}^{ \pm}+(Z \rightarrow \ell \ell) .
\end{array}
$$

We choose charged Higgs decays with high branching ratio to electroweakino pairs with the long-lived $\widetilde{\chi}_{1}^{ \pm}$. These events are triggered with the $\mathrm{Z}$ decay products arising from the decay of electroweakinos. There should be exactly two same flavour opposite sign leptons with 


\begin{tabular}{|c|c|c|c|}
\hline \multirow{2}{*}{ Trigger Z } & $\begin{array}{c}m_{H^{ \pm}} \\
(\mathrm{GeV})\end{array}$ & $\begin{array}{r}\text { Total event yield from the processes in } \\
\text { equation }(5.7) \text { at } 3 \mathrm{ab}^{-1}\end{array}$ \\
\cline { 3 - 4 } & & before Trigger Z & after Trigger Z \\
\hline$p_{T, \ell_{1,2}}>25 \mathrm{GeV},\left|\eta_{\ell_{1,2}}\right|<2.5$, & 620.29 & 427.04 & 173.12 \\
\cline { 3 - 4 } $76 \mathrm{GeV}<m_{\ell \ell}<106 \mathrm{GeV}$, & 819.77 & 262.40 & 119.54 \\
\cline { 2 - 4 }$p_{T, b}>30 \mathrm{GeV},\left|\eta_{b}\right|<2.5$ & 1077.18 & 101.91 & 47.98 \\
\hline
\end{tabular}

Table 30. The event yield at $3 \mathrm{ab}^{-1}$ from all the processes before and after, applying trigger cuts and $p_{T, \widetilde{\chi}_{1}^{ \pm}}>100 \mathrm{GeV},\left|\eta_{\widetilde{\chi}_{1}^{ \pm}}\right|<2.5$.

\begin{tabular}{|c|c||c|c|c|c|c|c|}
\hline$m_{H^{ \pm}}$ & \multicolumn{2}{|c||}{$\beta c \gamma \tau$ of } & \multicolumn{6}{|c|}{ Fraction of events after Trigger Z in \% within } \\
\cline { 3 - 8 }$(\mathrm{GeV})$ & $\tilde{\chi}_{1}^{ \pm}(\mathrm{cm})$ & $0-3 \mathrm{~mm}$ & $3-30 \mathrm{~mm}$ & $30 \mathrm{~mm}-10 \mathrm{~cm}$ & $10-30 \mathrm{~cm}$ & $30-100 \mathrm{~cm}$ & $>100 \mathrm{~cm}$ \\
\hline \hline 620.29 & 27.66 & 0.91 & 6.90 & 16.22 & 31.31 & 36.09 & 8.57 \\
\hline 819.77 & 18.10 & 0.90 & 7.94 & 16.97 & 32.75 & 33.86 & 7.58 \\
\hline 1077.18 & 3.7 & 4.07 & 28.75 & 37.55 & 24.98 & 4.60 & 0.05 \\
\hline
\end{tabular}

Table 31. The fractional number of events which decay at different parts inside tracker for the $4 F$ production process with $H^{ \pm} \rightarrow \widetilde{\chi}_{2}^{ \pm} \widetilde{\chi}_{1}^{0}$ in $\ell \ell+$ LLCP category.

$p_{T}>25 \mathrm{GeV}$ and $|\eta|<2.5$. The di-lepton invariant mass should be within $15 \mathrm{GeV}$ window around $Z$ boson mass. We also demand at least one b-tagged jet with $p_{T}>30 \mathrm{GeV}$ and $|\eta|<2.5$. In addition, the long-lived charged track must be within $|\eta|<2.5$ with $p_{T}>100 \mathrm{GeV}$. Table 30 summarises these trigger cuts along with the number of events at $3 a b^{-1}$, before and after the cuts. We also calculate the decay length and the decay fractions of the LLCP within different parts of the tracker for all the benchmark points, which are shown in table 31.

\subsection{2 $b \bar{b}+$ LLCP}

In this case we consider the following charged Higgs decay cascades:

$$
\begin{array}{ll}
H^{ \pm} \rightarrow \tilde{\chi}_{1}^{ \pm} \tilde{\chi}_{2,3}^{0}, & \tilde{\chi}_{2,3}^{0} \rightarrow \tilde{\chi}_{1}^{0}+(h \rightarrow b \bar{b}), \\
H^{ \pm} \rightarrow \widetilde{\chi}_{2}^{ \pm} \widetilde{\chi}_{1}^{0}, & \tilde{\chi}_{2}^{ \pm} \rightarrow \widetilde{\chi}_{1}^{ \pm}+(h \rightarrow b \bar{b}) .
\end{array}
$$

This channel has the advantage of having higher event yield because of large $h \rightarrow b \bar{b}$ branching ratio but may also suffer from huge QCD backgrounds. Since the b-jets will have smearing effect from the detector at collider, we simulate the detector effect with Delphes-3.4.1 with the same configuration as discussed in section 4. The events should contain atleast two b-jets with $p_{T}>30 \mathrm{GeV}$ and $|\eta|<2.5$. Since the b-jets are coming from the SM Higgs boson, the invariant mass of the two b-jets must be in the range, $[90,130] \mathrm{GeV}$ with the separation in the $\eta-\phi$ plane as $\Delta R_{b b}=[0.4,2.0]$. As before, we allow only those LLCP track which are within $|\eta|<2.5$ with $p_{T}>100 \mathrm{GeV}$. We show these trigger cuts along with the event yield at $3 a b^{-1}$ in table 32 . In table 33, we list the fractions of charginos which decay at different ranges inside tracker. 


\begin{tabular}{|c|c|c|c|}
\hline \multirow{2}{*}{ Trigger hbb } & $\begin{array}{c}m_{H^{ \pm}} \\
(\mathrm{GeV})\end{array}$ & $\begin{array}{r}\text { Total event yield from the processes in } \\
\text { equation }(5.8) \text { at } 3 \mathrm{ab}^{-1}\end{array}$ \\
\cline { 3 - 4 } & & before Trigger hbb & after Trigger hbb \\
\hline$p_{T, b_{1,2}}>30 \mathrm{GeV},\left|\eta_{b_{1,2}}\right|<2.5$, & 620.29 & 3060.04 & 238.06 \\
\cline { 2 - 4 } $90 \mathrm{GeV}<m_{b b}<130 \mathrm{GeV}$, & 819.77 & 1801.37 & 169.75 \\
\cline { 2 - 4 } $0.4<\Delta R_{b b}<2.0$ & 1077.18 & 649.55 & 68.58 \\
\hline
\end{tabular}

Table 32. Summarising the trigger cuts (additional cut: $p_{T, \widetilde{\chi}_{1}^{ \pm}}>100 \mathrm{GeV}$ and $\left|\eta_{\widetilde{\chi}_{1}^{ \pm}}\right|<2.5$ ) and the event yield at $3 \mathrm{ab}^{-1}$ in the $b \bar{b}+$ LLCP category.

\begin{tabular}{|c|c||c|c|c|c|c|c|}
\hline$m_{H^{ \pm}}$ & \multicolumn{2}{|c||}{$\beta c \gamma \tau$ of } & \multicolumn{6}{c|}{ Fraction of events after Trigger hbb in \% within } \\
\cline { 4 - 8 }$(\mathrm{GeV})$ & $\tilde{\chi}_{1}^{ \pm}(\mathrm{cm})$ & $0-3 \mathrm{~mm}$ & $3-30 \mathrm{~mm}$ & $30 \mathrm{~mm}-10 \mathrm{~cm}$ & $10-30 \mathrm{~cm}$ & $30-100 \mathrm{~cm}$ & $>100 \mathrm{~cm}$ \\
\hline \hline 620.29 & 27.66 & 6.43 & 7.86 & 16.55 & 31.49 & 32.08 & 5.59 \\
\hline 819.77 & 18.10 & 6.09 & 8.96 & 17.59 & 31.27 & 30.00 & 6.09 \\
\hline 1077.18 & 3.7 & 10.55 & 31.13 & 34.07 & 20.67 & 3.54 & 0.04 \\
\hline
\end{tabular}

Table 33. The fractional number of events which decay at different parts inside tracker for the $4 F$ production process with $H^{ \pm} \rightarrow \widetilde{\chi}_{2}^{ \pm} \widetilde{\chi}_{1}^{0}$ in $b \bar{b}+$ LLCP category.

\subsection{3 $\gamma \gamma+$ LLCP}

Finally we consider the following decay chain of the charged Higgs:

$$
\begin{aligned}
& H^{ \pm} \rightarrow \tilde{\chi}_{1}^{ \pm} \tilde{\chi}_{2,3}^{0}, \quad \tilde{\chi}_{2,3}^{0} \rightarrow \widetilde{\chi}_{1}^{0}+(h \rightarrow \gamma \gamma), \\
& H^{ \pm} \rightarrow \tilde{\chi}_{2}^{ \pm} \tilde{\chi}_{1}^{0}, \quad \tilde{\chi}_{2}^{ \pm} \rightarrow \tilde{\chi}_{1}^{ \pm}+(h \rightarrow \gamma \gamma) .
\end{aligned}
$$

This is the cleanest channel because of the photons in the final state at the cost of event yield. In this channel, we demand exactly two photons and at least one b-tagged jet with $p_{T}>30 \mathrm{GeV}$ and $|\eta|<2.5$. The di-photon invariant mass must fall in the range, $[122,128] \mathrm{GeV}$ with the $\Delta R$ separation between the photons, $\Delta R_{\gamma \gamma}=[0.4,2.0]$. Here, the event yield (table 34 ) is negligible even at $14 \mathrm{TeV}$ with $3 \mathrm{ab}^{-1}$ of integrated luminosity. We would like to mention here that the matched cross-sections in table 29 becomes $113.84 \mathrm{fb}$, $75.50 \mathrm{fb}$ and $38.44 \mathrm{fb}$ for $m_{H^{ \pm}}=620.29,819.77$, and $1077.18 \mathrm{GeV}$ respectively at the proposed HE-LHC (High Energy LHC) with $\sqrt{s}=27 \mathrm{TeV}$. This will increase the event yield an order of magnitude higher to have a better prospect of observing charged Higgs in this channel. We show the decay fractions of charginos at different track ranges in table 35 .

Summary LLCP final states. To summarise this section, we find possible interesting signatures for discovering heavy neutral and charged Higgs boson at the collider. Because of the boost received from the heavy Higgs decay, the charginos can travel larger distance inside the detector compared to their decay length. This can improve the existing sensitivity on disappearing track searches and look for possible signature of heavy Higgs production. If the decay length of long-lived particle is less than $1 \mathrm{~cm}$ or a few $\mathrm{mm}$, a short track is formed, called tracklet. Their search has been proposed in the literature [163, 174-176]. ${ }^{16}$ In case

\footnotetext{
${ }^{16}$ In case of disappearing track, one has to measure the lifetime of long-lived particle. This can be looked up for example in ref. [177] and the references therein.
} 


\begin{tabular}{|c|c|c|c|}
\hline \multirow{2}{*}{ Trigger $h \gamma \gamma$} & $\begin{array}{c}m_{H^{ \pm}} \\
(\mathrm{GeV})\end{array}$ & $\begin{array}{c}\text { Total event yield from the processes in } \\
\text { equation }(5.9) \text { at } 3 \mathrm{ab}^{-1}\end{array}$ \\
\cline { 3 - 4 } & & $\begin{array}{c}\text { before Trigger } h \gamma \gamma \\
\text { after Trigger } h \gamma \gamma\end{array}$ \\
\hline$p_{T, \gamma_{1,2}, b}>30 \mathrm{GeV},\left|\eta_{\gamma_{1,2}, b}\right|<2.5$, & 620.29 & 11.93 & 3.65 \\
\cline { 3 - 4 } $122 \mathrm{GeV}<m_{\gamma \gamma}<128 \mathrm{GeV}$, & 819.77 & 7.03 & 2.38 \\
\cline { 3 - 4 } $0.4<\Delta R_{\gamma \gamma}<2.0$ & 1077.18 & 2.53 & 0.87 \\
\hline
\end{tabular}

Table 34. Summarising the trigger cuts (additional cuts applied on $\widetilde{\chi}_{1}^{ \pm}: p_{T, \tilde{\chi}_{1}^{ \pm}}>100 \mathrm{GeV}$ and $\left.\left|\eta_{\widetilde{\chi}_{1}^{ \pm}}\right|<2.5\right)$ and the event yield at $3 \mathrm{ab}^{-1}$ in the $\gamma \gamma+$ LLCP category.

\begin{tabular}{|c|c||c|c|c|c|c|c|}
\hline$m_{H^{ \pm}}$ & \multicolumn{1}{|c|}{$\beta c \gamma \tau$ of } & \multicolumn{6}{|c|}{ Fraction of events after Trigger $h \gamma \gamma$ in \% within } \\
\cline { 3 - 8 }$(\mathrm{GeV})$ & $\tilde{\chi}_{1}^{ \pm}(\mathrm{cm})$ & $0-3 \mathrm{~mm}$ & $3-30 \mathrm{~mm}$ & $30 \mathrm{~mm}-10 \mathrm{~cm}$ & $10-30 \mathrm{~cm}$ & $30-100 \mathrm{~cm}$ & $>100 \mathrm{~cm}$ \\
\hline \hline 620.29 & 27.66 & 0.89 & 7.71 & 16.30 & 31.60 & 35.56 & 7.94 \\
\hline 819.77 & 18.10 & 0.90 & 8.22 & 17.46 & 31.95 & 34.28 & 7.19 \\
\hline 1077.18 & 3.7 & 3.93 & 29.98 & 37.70 & 24.41 & 3.94 & 0.04 \\
\hline
\end{tabular}

Table 35. The fractional number of events which decay at different parts inside tracker for the $4 F$ production process with $H^{ \pm} \rightarrow \widetilde{\chi}_{2}^{ \pm} \widetilde{\chi}_{1}^{0}$ in $\gamma \gamma+$ LLCP category.

of charged Higgs production, one can look for $\ell \ell+\mathbb{E}_{T}, b \bar{b}+\mathbb{E}_{T}$ and $\gamma \gamma+\mathbb{E}_{T}$ final states along with a disappearing charged track with large transverse momentum. Background contamination to these final states mainly comes in the form of fake track signature due to incorrect reconstruction of hits inside tracker, along with SM particles giving rise to similar final states. Data-driven techniques could play an important role in estimating these backgrounds which is beyond the scope of our work. Instead we compute the event yield for such signal processes at the HL-LHC and calculate the fraction of events which decay at different parts of the tracker. In short, the features of these final states opens a new avenue to search for heavy Higgses at the collider.

\section{Conclusion}

In this work, we visited the decays to heavy Higgs to supersymmetric particles, particularly concentrating on the electroweakino sector. After performing a survey of available MSSM parameter space, we chose a few benchmark scenarios and analysed in detail the reach of HL-LHC for these benchmark in the mono-X category, particularly concentrating on mono-Z (dilepton) and mono-h $(b \bar{b}, \gamma \gamma)$ final states. These were further split into analyses depending on the Higgs production mechanisms which corresponded to b-tag or b-veto category analysis. This resulted in a total of six analyses categories.

In order to perform signal optimisation, we considered events originating from SM backgrounds as well as direct susy production at the LHC. We demonstrated by constructing specific kinematic variables, that it is possible to discriminate between direct electroweakino production and electroweakino production via Higgs decays. Such discrimination relies on the fact that electroweakino production via heavy Higgs decays carries 
an imprint of the resonance, while the direct electroweakino production takes place via off-shell SM mediators.

In terms of optimised analysis, we get the largest significances for the dilepton + MET final state due to the cleanliness of the signal at the LHC. Within this category, we demonstrate that exploiting additional b-tagged jet helps improve the significance of the signal. The second most important channel is the $b \bar{b}+\mathbb{E}_{T}$ final state, which results in the mono-h events. In this case, tagging the additional jet actually reduces the significance due to increased backgrounds. The least promising final state is the $\gamma \gamma+\mathbb{E}_{T}$ final state. This is understandable as the SM Higgs to photon branching ratio is very small, therefore even if the channel is clean, it is not helpful at the LHC.

Complementing our analysis in the missing energy final state, we also explored the possibility of heavy Higgs decays to long lived chargino. These chargino travel a finite distance in the detector before decaying, therefore producing the disappearing track signature. Heavy Higgs decays to long lived chargino can involve visible states along with disappearing track in the detector e.g. jets or leptons. We demonstrate that boost gained by the chargino due to on-shell Higgs mediator might be exploited for such searches potentially increasing the reach of LHC searches for disappearing track analyses in addition opening another channel for heavy Higgs searches.

While the existence of susy at the LHC is increasingly being doubted, all attempts should be made to search for a possible signature before abandoning the idea of SUSY. With this in mind, the heavy Higgs decays to susy present an interesting opportunity to search for new physics scenarios at the HL-LHC.

\section{A Random scan results}

In this appendix, we discuss the results of our flat random scan in the plane of various MSSM parameters. In figure 24, we show different mass planes to this effect. They are $m_{A}-\tan \beta$ (top left), $\Omega h^{2}-M_{\widetilde{\chi}_{1}^{0}}$ where $\Omega h^{2}$ is the relic abundance of dark matter (top right), $\mu-M_{2}$ (middle left), $M_{\widetilde{\chi}_{1}^{ \pm}}-M_{\widetilde{\chi}_{1}^{0}}$ (middle right), $M_{\tilde{b_{1}}}-M_{\tilde{t_{1}}}$ (bottom left) and $M_{\tilde{b_{2}}}-M_{\tilde{t_{2}}}$ (bottom right) plane. We plot the allowed points after each of the experimental constraints i.e. SM Higgs boson mass range $(122,128) \mathrm{GeV}$, LEP constraints (grey), the flavour physics constraint (blue), Higgs signal strengths and heavy Higgs searches (green), dark matter direct detection constraint (yellow) and finally LHC constraints (red). The exact codes tools and experimental constraints used here, has been mentioned in section 3 , In general there are a few take home messages here. First, we see that in general points with light pseudo-scalar Higgs are ruled out primarily by the heavy Higgs searches in combination with the SM Higgs signal strength measurements. Second, in general light electroweakinos are in general allowed at the LHC, such points are either strongly winolike or strongly higgsino-like if at least one of the electroweakino is to be light. For heavier electroweakinos in general an arbitrary combination can be obtained. Such an observation has important consequences at the LHC, as a wino-like LSP is often accompanied with a long lived chargino. This is reflected in our benchmark points. Finally, we also see that generally it is difficult to obtain light stop and sbottoms at the LHC. 

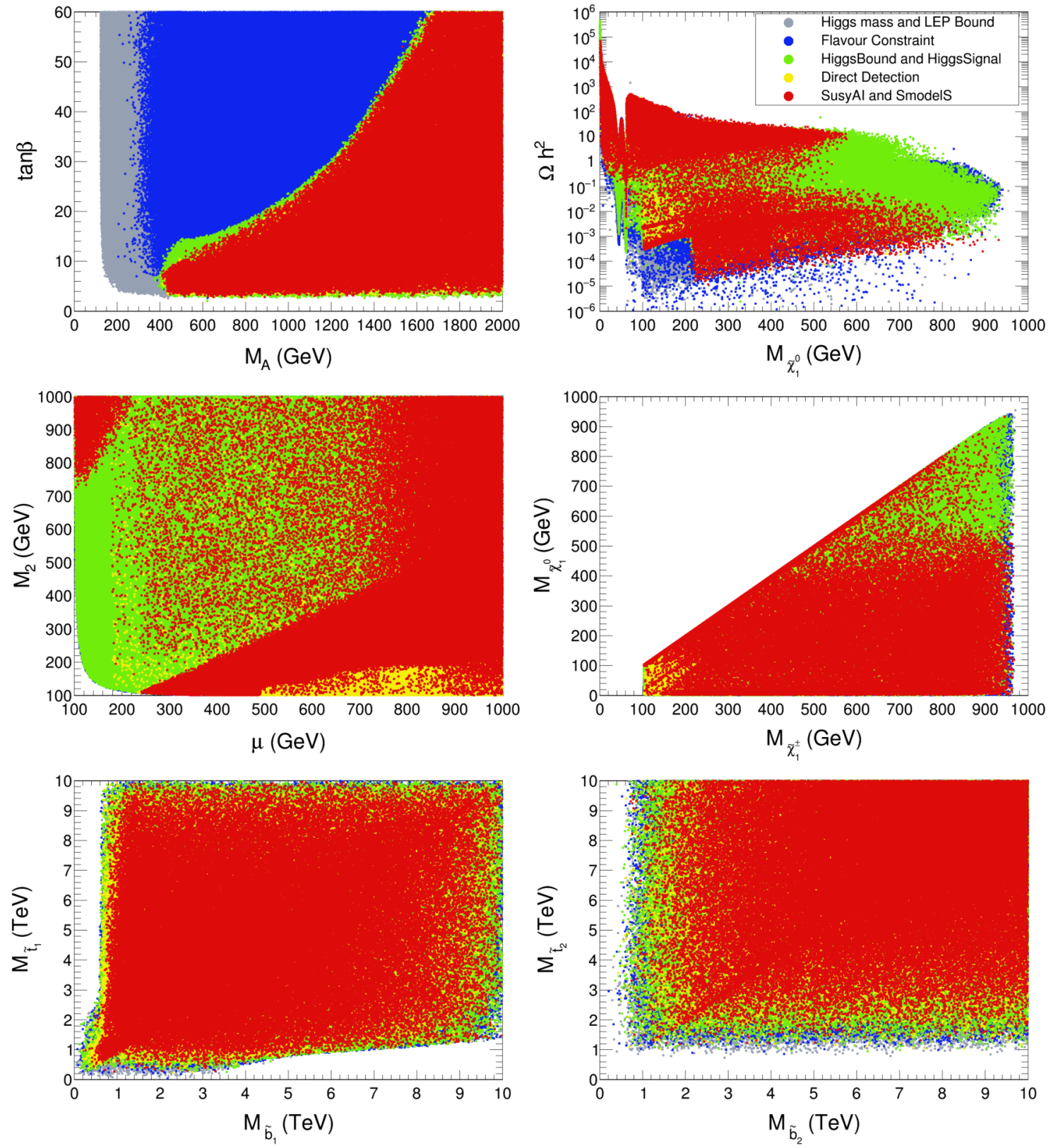

Figure 24. Scatter plots in the plane of various MSSM parameters and masses. 


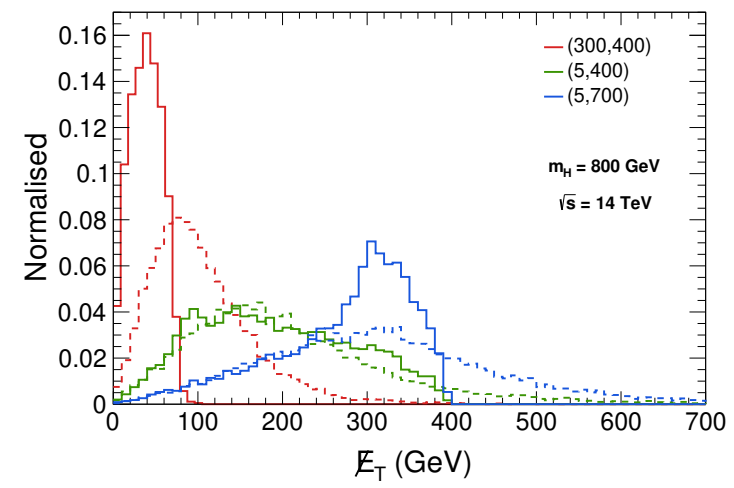

Figure 25. Normalised distribution of $\mathbb{E}_{T}$ at the parton level. The other details are same as the left plot of figure 10 .

\section{B Parton level kinematics}

At the parton level, the heavy Higgs is produced almost at rest in case of resonant production and the leptons from $\mathrm{Z}$ boson gets boost only from the mass difference between neutralinos $\left(m_{\widetilde{\chi}_{2}^{0}}-m_{\widetilde{\chi}_{1}^{0}}\right)$. In case of direct production, the neutralinos are produced with large transverse momentum $\left(p_{T}\right)$ which goes into the final state leptons along with the contribution coming from the mass gap between neutralinos. Therefore, the direct susy production creates more missing transverse momentum $\left(\mathbb{E}_{T}\right)$ compared to the resonant production. Figure 25 describes this feature which has an endpoint for the resonant susy production. Now, at the detector level the whole system of neutralinos gets recoiled against jets. The heavy Higgs in the resonant production is now produced with some boost as compared with parton level case and give similar $\mathbb{E}_{T}$ distribution from direct susy production for $\left(m_{\widetilde{\chi}_{2}^{0}}, m_{\widetilde{\chi}_{1}^{0}}\right)=(400,300)$ and $(400,5) \mathrm{GeV}$ cases which is depicted in figure 10. 


\section{Summarising the cross sections and generator level cuts for the SM backgrounds}

Here, we give a summary of the production cross section of the SM backgrounds and the cuts used while generating these backgrounds in MadGraph-2.6.5.

\begin{tabular}{|c|c|c|c|}
\hline Process & Backgrounds & $\begin{array}{l}\text { Generation-level cuts }\left(\ell=e^{ \pm}, \mu^{ \pm}, \tau^{ \pm}\right) \\
\text {(NA: Not Applied) }\end{array}$ & Cross section $(\mathrm{fb})$ \\
\hline \multicolumn{4}{|c|}{$p p \rightarrow H \rightarrow \widetilde{\chi}_{1}^{0}+\left(\widetilde{\chi}_{2,3}^{0}\right) \rightarrow \widetilde{\chi}_{1}^{0}+\left(\widetilde{\chi}_{1}^{0}+Z, Z \rightarrow \ell \ell\right)$ final state } \\
\hline \multirow{9}{*}{$\ell \ell+\mathbb{E}_{T}$} & $Z Z+$ jets & $\begin{array}{c}p_{T, j / b}>20 \mathrm{GeV},\left|\eta_{j}\right|<5.0,\left|\eta_{b}\right|<3.0 \\
\Delta R_{b, j},{ }^{17}>0.2\end{array}$ & 11427.77 \\
\hline & $W Z+$ jets & same as $Z Z+$ jets & 39684.46 \\
\hline & $V V V$ & NA & 266.61 \\
\hline & $t \bar{t} Z$ & NA & 851.86 \\
\hline & $t \bar{t}$ leptonic & $\begin{aligned} p_{T, j / b} & >20 \mathrm{GeV}, p_{T, \ell}>15 \mathrm{GeV},\left|\eta_{j}\right|<5.0 \\
\left|\eta_{b / \ell}\right| & <3.0, \Delta R_{b, j, \ell}>0.2, \mathbb{E}_{T}>100 \mathrm{GeV}\end{aligned}$ & 12013.93 \\
\hline & $\ell \ell+$ jets & same as $t \bar{t}$ leptonic & 3154714.02 \\
\hline & $t \bar{t} h$ & NA & 611.30 \\
\hline & $t \bar{t} W$ & NA & 520.03 \\
\hline & $W W+$ jets & $\begin{array}{c}p_{T, j / b}>20 \mathrm{GeV},\left|\eta_{j}\right|<5.0,\left|\eta_{b}\right|<3.0 \\
\Delta R_{b, j}>0.2\end{array}$ & 478670.32 \\
\hline
\end{tabular}

Table 36. Generation level cuts and cross-sections for the various Standard Model backgrounds used in the analyses.

\footnotetext{
${ }^{17} \Delta R_{b, j}$ means $\Delta R$ between all possible combination of $b$ and light jet.
} 


\begin{tabular}{|c|c|c|c|}
\hline Process & Backgrounds & $\begin{array}{c}\text { Generation-level cuts }\left(\ell=e^{ \pm}, \mu^{ \pm}, \tau^{ \pm}\right) \\
\text {(NA: Not Applied) }\end{array}$ & Cross section $(\mathrm{fb})$ \\
\hline \multicolumn{4}{|c|}{$p p \rightarrow H \rightarrow \widetilde{\chi}_{1}^{0}+\widetilde{\chi}_{2,3}^{0} \rightarrow \widetilde{\chi}_{1}^{0}+\left(\widetilde{\chi}_{1}^{0}+h, h \rightarrow b \bar{b} / \gamma \gamma\right)$ final states } \\
\hline \multirow{9}{*}{$b \bar{b}+\mathbb{E}_{T}$} & $t \bar{t}$ hadronic & $\begin{array}{c}p_{T, j / b}>20 \mathrm{GeV},\left|\eta_{j}\right|<5.0,\left|\eta_{b}\right|<3.0 \\
\Delta R_{b, j, \ell}>0.2, m_{b b}>30 \mathrm{GeV}, \mathbb{E}_{T}>100 \mathrm{GeV}\end{array}$ & 165994.96 \\
\hline & $t \bar{t}$ semi-leptonic & same as $t \bar{t}$ hadronic & 32282.76 \\
\hline & $t \bar{t}$ leptonic & same as $t \bar{t}$ hadronic & 16340.61 \\
\hline & $Z b \bar{b}, Z \rightarrow \nu \nu$ & $\begin{array}{c}p_{T, b}>20 \mathrm{GeV},\left|\eta_{b}\right|<3.0, \Delta R_{b b}>0.2 \\
m_{b b}>30 \mathrm{GeV}, \notin_{T}>100 \mathrm{GeV}\end{array}$ & 2158.69 \\
\hline & $Z h+$ jets & $\begin{array}{c}p_{T, j / b}>20 \mathrm{GeV},\left|\eta_{j}\right|<5.0,\left|\eta_{b}\right|<3.0 \\
\Delta R_{j, b}>0.2\end{array}$ & 969.00 \\
\hline & $W h+$ jet, $W \rightarrow \ell \nu, h \rightarrow b \bar{b}$ & same as $t \bar{t}$ hadronic & 0.55 \\
\hline & $t \bar{t} h$ & NA & 611.30 \\
\hline & $t \bar{t} Z$ & NA & 851.86 \\
\hline & $t \bar{t} W$ & $\mathrm{NA}$ & 520.03 \\
\hline \multirow{4}{*}{$\gamma \gamma+\mathbb{E}_{T}$} & $Z h+$ jet, $h \rightarrow \gamma \gamma$ & $\begin{array}{l}p_{T, j / b / \gamma}>20 \mathrm{GeV},\left|\eta_{j}\right|<5.0,\left|\eta_{b / \gamma}\right|<3.0 \\
\Delta R_{j / b / \gamma}>0.2,110 \mathrm{GeV}<m_{\gamma \gamma}<140 \mathrm{GeV}\end{array}$ & 1.65 \\
\hline & $W h+$ jet, $h \rightarrow \gamma \gamma$ & same as $Z h+$ jet & 2.54 \\
\hline & $Z \gamma \gamma+$ jet, $Z \rightarrow \nu \nu$ & same as $Z h+$ jet & 1.87 \\
\hline & $t \bar{t} h, h \rightarrow \gamma \gamma$ & same as $Z h+$ jet & 1.19 \\
\hline
\end{tabular}

Table 37. Generation level cuts and cross-sections for the various Standard Model backgrounds used in the analyses.

\section{Acknowledgments}

We thank Rahool kumar Barman, Aravind Vijay, Alberto Escalante del Valle, Nishita Desai, Jean-Loic Kneur and Dirk Zerwas for helpful discussions at various stages of the work. The work of BB is supported by the Department of Science and Technology, Government of India, under the Grant Agreement number IFA13-PH-75 (INSPIRE Faculty Award). The work of RMG is supported by the Department of Science and Technology, India under Grant No. SR/S2/JCB-64/2007. The work of Najimuddin Khan is supported by the Department of Science and Technology, Government of INDIA under the SERB-Grant PDF/2017/00372. SK is supported by Elise-Richter grant project number V592-N27. We acknowledge Austrian-India WTZ-DAE exchange project number IN 15/2018. S.K. thanks IISc CHEP department for hospitality and WHEPP workshop, IIT Guwahati for hospitality where part of this work was completed.

Open Access. This article is distributed under the terms of the Creative Commons Attribution License (CC-BY 4.0), which permits any use, distribution and reproduction in any medium, provided the original author(s) and source are credited. 


\section{References}

[1] R.G.M. Drees and P. Roy, Theory and Phenomenology of Sparticles, World Scientific, (2004).

[2] H. Baer and X. Tata, Weak Scale Supersymmetry, Cambridge University Press, (2006).

[3] S.P. Martin, A supersymmetry primer, Adv. Ser. Direct. High Energy Phys. 18 (1998) 1 [hep-ph/9709356] [INSPIRE].

[4] A. Djouadi, The anatomy of electro-weak symmetry breaking. II. The Higgs bosons in the minimal supersymmetric model, Phys. Rept. 459 (2008) 1 [hep-ph/0503173] [InSPIRE].

[5] ATLAS collaboration, Search for additional heavy neutral Higgs and gauge bosons in the ditau final state produced in $36 \mathrm{fb}^{-1}$ of pp collisions at $\sqrt{\mathrm{s}}=13 \mathrm{TeV}$ with the ATLAS detector, JHEP 01 (2018) 055 [arXiv:1709.07242] [INSPIRE].

[6] CMS collaboration, Search for Higgs boson pair production in the final state containing two photons and two bottom quarks in proton-proton collisions at $\sqrt{s}=13 \mathrm{TeV}$, Tech. Rep. CMS-PAS-HIG-17-008, CERN, Geneva (2017).

[7] CMS collaboration, Search for resonant pair production of Higgs bosons decaying to bottom quark-antiquark pairs in proton-proton collisions at $13 \mathrm{TeV}$, Tech. Rep. CMS-PAS-HIG-17-009, CERN, Geneva (2017).

[8] CMS collaboration, Search for Higgs boson pair production in events with two bottom quarks and two tau leptons in proton-proton collisions at $\sqrt{s}=13$ TeV, Phys. Lett. B 778 (2018) 101 [arXiv:1707.02909] [INSPIRE].

[9] ATLAS collaboration, Search for Heavy Higgs Bosons A/H Decaying to a Top Quark Pair in pp Collisions at $\sqrt{s}=8 \mathrm{TeV}$ with the ATLAS Detector, Phys. Rev. Lett. 119 (2017) 191803 [arXiv:1707.06025] [INSPIRE].

[10] ATLAS collaboration, Search for heavy particles decaying into top-quark pairs using lepton-plus-jets events in proton-proton collisions at $\sqrt{s}=13$ TeV with the ATLAS detector, Eur. Phys. J. C 78 (2018) 565 [arXiv: 1804.10823] [INSPIRE].

[11] A. Adhikary, S. Banerjee, R. Kumar Barman and B. Bhattacherjee, Resonant heavy Higgs searches at the HL-LHC, JHEP 09 (2019) 068 [arXiv: 1812.05640] [INSPIRE].

[12] M. Bisset, J. Li and N. Kersting, How to Detect 'Decoupled' Heavy Supersymmetric Higgs Bosons, arXiv:0709.1031 [INSPIRE].

[13] M. Bisset, J. Li, N. Kersting, R. Lu, F. Moortgat and S. Moretti, Four-lepton LHC events from MSSM Higgs boson decays into neutralino and chargino pairs, JHEP 08 (2009) 037 [arXiv:0709.1029] [INSPIRE].

[14] A. Arhrib, R. Benbrik, M. Chabab and C.-H. Chen, Pair production of neutralinos and charginos at the LHC: the role of Higgs bosons exchange, Phys. Rev. D 84 (2011) 115012 [arXiv:1109.0518] [INSPIRE].

[15] J.F. Gunion et al., Decays of Higgs Bosons to Neutralinos and Charginos in the Minimal Supersymmetric Model: Calculation and Phenomenology, Int. J. Mod. Phys. A 2 (1987) 1035 [INSPIRE].

[16] J.F. Gunion and H.E. Haber, Higgs Bosons in Supersymmetric Models. 3. Decays Into Neutralinos and Charginos, Nucl. Phys. B 307 (1988) 445 [Erratum ibid. 402 (1993) 569] [INSPIRE]. 
[17] A. Djouadi, P. Janot, J. Kalinowski and P.M. Zerwas, SUSY decays of Higgs particles, Phys. Lett. B 376 (1996) 220 [hep-ph/9603368] [INSPIRE].

[18] G. Bélanger, F. Boudjema, F. Donato, R. Godbole and S. Rosier-Lees, SUSY Higgs at the LHC: Effects of light charginos and neutralinos, Nucl. Phys. B 581 (2000) 3 [hep-ph/0002039] [INSPIRE].

[19] M. Bisset, M. Guchait and S. Moretti, Signatures of MSSM charged Higgs bosons via chargino neutralino decay channels at the LHC, Eur. Phys. J. C 19 (2001) 143 [hep-ph/0010253] [INSPIRE].

[20] S.Y. Choi, M. Drees, J.S. Lee and J. Song, Supersymmetric Higgs boson decays in the MSSM with explicit CP-violation, Eur. Phys. J. C 25 (2002) 307 [hep-ph/0204200] [InSPIRE].

[21] C. Charlot, R. Salerno and Y. Sirois, Observability of the heavy neutral SUSY Higgs bosons decaying into neutralinos, J. Phys. G 34 (2007) N1 [INSPIRE].

[22] T. Li, Decoupling MSSM Higgs Sector and Heavy Higgs Decay, Phys. Lett. B 728 (2014) 77 [arXiv:1309.6713] [INSPIRE].

[23] G. Bélanger, D. Ghosh, R. Godbole and S. Kulkarni, Light stop in the MSSM after LHC Run 1, JHEP 09 (2015) 214 [arXiv: 1506.00665] [InSPIRE].

[24] B. Ananthanarayan, J. Lahiri and P.N. Pandita, Invisible decays of the heavier Higgs boson in the minimal supersymmetric standard model, Phys. Rev. D 91 (2015) 115025 [arXiv: 1507.01747] [INSPIRE].

[25] A. Djouadi, L. Maiani, A. Polosa, J. Quevillon and V. Riquer, Fully covering the MSSM Higgs sector at the LHC, JHEP 06 (2015) 168 [arXiv: 1502.05653] [INSPIRE].

[26] R.K. Barman, B. Bhattacherjee, A. Chakraborty and A. Choudhury, Study of MSSM heavy Higgs bosons decaying into charginos and neutralinos, Phys. Rev. D 94 (2016) 075013 [arXiv: 1607.00676] [INSPIRE].

[27] S. Gori, Z. Liu and B. Shakya, Heavy Higgs as a Portal to the Supersymmetric Electroweak Sector, JHEP 04 (2019) 049 [arXiv: 1811.11918] [INSPIRE].

[28] S. Baum, N.R. Shah and K. Freese, The NMSSM is within Reach of the LHC: Mass Correlations \& Decay Signatures, JHEP 04 (2019) 011 [arXiv: 1901.02332] [INSPIRE].

[29] S. Profumo, T. Stefaniak and L. Stephenson Haskins, The Not-So-Well Tempered Neutralino, Phys. Rev. D 96 (2017) 055018 [arXiv:1706.08537] [INSPIRE].

[30] E. Bagnaschi et al., MSSM Higgs Boson Searches at the LHC: Benchmark Scenarios for Run 2 and Beyond, Eur. Phys. J. C 79 (2019) 617 [arXiv:1808.07542] [INSPIRE].

[31] H. Bahl, S. Liebler and T. Stefaniak, MSSM Higgs benchmark scenarios for Run 2 and beyond: the low $\tan \beta$ region, Eur. Phys. J. C 79 (2019) 279 [arXiv:1901. 05933] [inSPIRE].

[32] CMS collaboration, Search for new physics in events with a leptonically decaying $Z$ boson and a large transverse momentum imbalance in proton-proton collisions at $\sqrt{s}=13 \mathrm{TeV}$, Eur. Phys. J. C $\mathbf{7 8}$ (2018) 291 [arXiv:1711.00431] [InSPIRE].

[33] G.F. Giudice, M.A. Luty, H. Murayama and R. Rattazzi, Gaugino mass without singlets, JHEP 12 (1998) 027 [hep-ph/9810442] [INSPIRE].

[34] L. Randall and R. Sundrum, Out of this world supersymmetry breaking, Nucl. Phys. B 557 (1999) 79 [hep-th/9810155] [INSPIRE].

[35] B.C. Allanach, A. Djouadi, J.L. Kneur, W. Porod and P. Slavich, Precise determination of the neutral Higgs boson masses in the MSSM, JHEP 09 (2004) 044 [hep-ph/0406166] [INSPIRE]. 
[36] A. Djouadi, J.-L. Kneur and G. Moultaka, SuSpect: A Fortran code for the supersymmetric and Higgs particle spectrum in the MSSM, Comput. Phys. Commun. 176 (2007) 426 [hep-ph/0211331] [INSPIRE].

[37] D. Chowdhury, R.M. Godbole, K.A. Mohan and S.K. Vempati, Charge and Color Breaking Constraints in MSSM after the Higgs Discovery at LHC, JHEP 02 (2014) 110 [Erratum ibid. 03 (2018) 149] [arXiv:1310.1932] [INSPIRE].

[38] T. Gherghetta, G.F. Giudice and J.D. Wells, Phenomenological consequences of supersymmetry with anomaly induced masses, Nucl. Phys. B 559 (1999) 27 [hep-ph/9904378] [INSPIRE].

[39] M. Ibe, S. Matsumoto and R. Sato, Mass Splitting between Charged and Neutral Winos at Two-Loop Level, Phys. Lett. B $\mathbf{7 2 1}$ (2013) 252 [arXiv:1212.5989] [INSPIRE].

[40] A.V. Gladyshev, D.I. Kazakov and M.G. Paucar, Long-lived Charginos in the Focus-point Region of the MSSM Parameter Space, J. Phys. G 36 (2009) 125009 [arXiv:0811.2911] [INSPIRE].

[41] S. Kulkarni and L. Lechner, Characterizing simplified models for heavy Higgs decays to supersymmetric particles, arXiv:1711.00056 [INSPIRE].

[42] A. Arbey, M. Battaglia and F. Mahmoudi, Supersymmetric Heavy Higgs Bosons at the LHC, Phys. Rev. D 88 (2013) 015007 [arXiv:1303.7450] [inSPIRE].

[43] XENON collaboration, Dark Matter Search Results from a One Ton-Year Exposure of XENON1T, Phys. Rev. Lett. 121 (2018) 111302 [arXiv:1805.12562] [INSPIRE].

[44] HFLAV collaboration, Averages of b-hadron, c-hadron, and $\tau$-lepton properties as of summer 2016, Eur. Phys. J. C $\mathbf{7 7}$ (2017) 895 [arXiv: 1612.07233] [INSPIRE].

[45] LHCb collaboration, Measurement of the $B_{s}^{0} \rightarrow \mu^{+} \mu^{-}$branching fraction and effective lifetime and search for $B^{0} \rightarrow \mu^{+} \mu^{-}$decays, Phys. Rev. Lett. 118 (2017) 191801 [arXiv: 1703. 05747] [INSPIRE].

[46] OPAL collaboration, Search for chargino and neutralino production at $\sqrt{s}=192 \mathrm{GeV}$ to $209 \mathrm{GeV}$ at LEP, Eur. Phys. J. C 35 (2004) 1 [hep-ex/0401026] [InSPIRE].

[47] G. Bélanger, F. Boudjema, A. Goudelis, A. Pukhov and B. Zaldivar, MicrOMEGAs5.0: Freeze-in, Comput. Phys. Commun. 231 (2018) 173 [arXiv:1801.03509] [InSPIRE].

[48] P. Bechtle et al., HiggsBounds-4: Improved Tests of Extended Higgs Sectors against Exclusion Bounds from LEP, the Tevatron and the LHC, Eur. Phys. J. C 74 (2014) 2693 [arXiv:1311.0055] [INSPIRE].

[49] P. Bechtle et al., Recent Developments in HiggsBounds and a Preview of HiggsSignals, PoS CHARGED2012 (2012) 024 [arXiv:1301.2345] [INSPIRE].

[50] P. Bechtle, O. Brein, S. Heinemeyer, G. Weiglein and K.E. Williams, HiggsBounds 2.0.0: Confronting Neutral and Charged Higgs Sector Predictions with Exclusion Bounds from LEP and the Tevatron, Comput. Phys. Commun. 182 (2011) 2605 [arXiv:1102.1898] [INSPIRE].

[51] P. Bechtle, O. Brein, S. Heinemeyer, G. Weiglein and K.E. Williams, HiggsBounds: Confronting Arbitrary Higgs Sectors with Exclusion Bounds from LEP and the Tevatron, Comput. Phys. Commun. 181 (2010) 138 [arXiv:0811.4169] [INSPIRE].

[52] P. Bechtle, S. Heinemeyer, O. Stål, T. Stefaniak and G. Weiglein, HiggsSignals: Confronting arbitrary Higgs sectors with measurements at the Tevatron and the LHC, Eur. Phys. J. C 74 (2014) 2711 [arXiv: 1305.1933] [INSPIRE]. 
[53] S. Caron, J.S. Kim, K. Rolbiecki, R. Ruiz de Austri and B. Stienen, The BSM-AI project: SUSY-AI-generalizing LHC limits on supersymmetry with machine learning, Eur. Phys. J. $C 77$ (2017) 257 [arXiv:1605.02797] [INSPIRE].

[54] S. Kraml et al., SModelS: a tool for interpreting simplified-model results from the LHC and its application to supersymmetry, Eur. Phys. J. C 74 (2014) 2868 [arXiv:1312.4175] [INSPIRE].

[55] F. Ambrogi et al., SModelS v1.1 user manual: Improving simplified model constraints with efficiency maps, Comput. Phys. Commun. 227 (2018) 72 [arXiv:1701.06586] [INSPIRE].

[56] F. Ambrogi et al., SModelS v1.2: long-lived particles, combination of signal regions, and other novelties, Comput. Phys. Commun. 251 (2020) 106848 [arXiv:1811.10624] [INSPIRE].

[57] J. Heisig, S. Kraml and A. Lessa, Constraining new physics with searches for long-lived particles: Implementation into SModelS, Phys. Lett. B 788 (2019) 87 [arXiv:1808.05229] [INSPIRE].

[58] J. Dutta, S. Kraml, A. Lessa and W. Waltenberger, SModelS extension with the CMS supersymmetry search results from Run 2, LHEP 1 (2018) 5 [arXiv: 1803.02204] [INSPIRE].

[59] ATLAS collaboration, Search for bottom squark pair production in proton-proton collisions at $\sqrt{s}=13 \mathrm{TeV}$ with the ATLAS detector, Eur. Phys. J. C 76 (2016) 547 [arXiv: 1606.08772] [INSPIRE].

[60] ATLAS collaboration, Search for top squarks in final states with one isolated lepton, jets, and missing transverse momentum in $\sqrt{s}=13 \mathrm{TeV}$ pp collisions with the ATLAS detector, Phys. Rev. D 94 (2016) 052009 [arXiv: 1606.03903] [InSPIRE].

[61] ATLAS collaboration, Search for squarks and gluinos in final states with jets and missing transverse momentum at $\sqrt{s}=13 \mathrm{TeV}$ with the ATLAS detector, Eur. Phys. J. C 76 (2016) 392 [arXiv: 1605.03814] [INSPIRE].

[62] ATLAS collaboration, Search for supersymmetry at $\sqrt{s}=13 \mathrm{TeV}$ in final states with jets and two same-sign leptons or three leptons with the ATLAS detector, Eur. Phys. J. C 76 (2016) 259 [arXiv: 1602.09058] [inSPIRE].

[63] ATLAS collaboration, Search for top squarks decaying to tau sleptons in pp collisions at $\sqrt{s}=13 \mathrm{TeV}$ with the ATLAS detector, Phys. Rev. D 98 (2018) 032008 [arXiv: 1803.10178] [INSPIRE].

[64] ATLAS collaboration, Search for supersymmetry in final states with charm jets and missing transverse momentum in $13 \mathrm{TeV}$ pp collisions with the ATLAS detector, JHEP 09 (2018) 050 [arXiv: 1805.01649] [inSPIRE].

[65] ATLAS collaboration, Search for new phenomena using the invariant mass distribution of same-flavour opposite-sign dilepton pairs in events with missing transverse momentum in $\sqrt{s}=13 \mathrm{TeV}$ pp collisions with the ATLAS detector, Eur. Phys. J. C 78 (2018) 625 [arXiv: 1805.11381] [INSPIRE].

[66] ATLAS collaboration, Search for chargino-neutralino production using recursive jigsaw reconstruction in final states with two or three charged leptons in proton-proton collisions at $\sqrt{s}=13 \mathrm{TeV}$ with the ATLAS detector, Phys. Rev. D 98 (2018) 092012 [arXiv: 1806. 02293] [INSPIRE].

[67] ATLAS collaboration, Search for strongly produced superpartners in final states with two same sign leptons with the ATLAS detector using $21 \mathrm{fb}-1$ of proton-proton collisions at $\sqrt{s}=8 \mathrm{TeV}$, Tech. Rep. ATLAS-CONF-2013-007, CERN, Geneva (Mar, 2013). 
[68] Search for strong production of supersymmetric particles in final states with missing transverse momentum and at least three b-jets using $20.1 \mathrm{fb}^{-1}$ of pp collisions at $\sqrt{\mathrm{s}}=8 \mathrm{TeV}$ with the ATLAS Detector, Tech. Rep. ATLAS-CONF-2013-061, CERN, Geneva (Jun, 2013).

[69] Search for strongly produced supersymmetric particles in decays with two leptons at $\sqrt{s}=8 \mathrm{TeV}$, Tech. Rep. ATLAS-CONF-2013-089, CERN, Geneva (Aug, 2013).

[70] ATLAS collaboration, Search for squarks and gluinos with the ATLAS detector in final states with jets and missing transverse momentum using $\sqrt{s}=8$ TeV proton-proton collision data, JHEP 09 (2014) 176 [arXiv:1405.7875] [INSPIRE].

[71] ATLAS collaboration, Search for new phenomena in final states with large jet multiplicities and missing transverse momentum at $\sqrt{s}=8 \mathrm{TeV}$ proton-proton collisions using the ATLAS experiment, JHEP 10 (2013) 130 [Erratum ibid. 01 (2014) 109] [arXiv:1308.1841] [INSPIRE].

[72] ATLAS collaboration, Search for direct third-generation squark pair production in final states with missing transverse momentum and two b-jets in $\sqrt{s}=8 \mathrm{TeV}$ pp collisions with the ATLAS detector, JHEP 10 (2013) 189 [arXiv:1308.2631] [INSPIRE].

[73] ATLAS collaboration, Search for direct top squark pair production in events with a $Z$ boson, b-jets and missing transverse momentum in $\sqrt{s}=8 \mathrm{TeV}$ pp collisions with the ATLAS detector, Eur. Phys. J. C $\mathbf{7 4}$ (2014) 2883 [arXiv:1403.5222] [INSPIRE].

[74] ATLAS collaboration, Search for supersymmetry at $\sqrt{s}=8 \mathrm{TeV}$ in final states with jets and two same-sign leptons or three leptons with the ATLAS detector, JHEP 06 (2014) 035 [arXiv: 1404.2500] [INSPIRE].

[75] ATLAS collaboration, Search for direct production of charginos, neutralinos and sleptons in final states with two leptons and missing transverse momentum in pp collisions at $\sqrt{s}=8 \mathrm{TeV}$ with the ATLAS detector, JHEP 05 (2014) 071 [arXiv:1403.5294] [INSPIRE].

[76] ATLAS collaboration, Search for direct production of charginos and neutralinos in events with three leptons and missing transverse momentum in $\sqrt{s}=8 \mathrm{TeV}$ pp collisions with the ATLAS detector, JHEP 04 (2014) 169 [arXiv:1402.7029] [INSPIRE].

[77] ATLAS collaboration, Search for top squark pair production in final states with one isolated lepton, jets, and missing transverse momentum in $\sqrt{s}=8 \mathrm{TeV}$ pp collisions with the ATLAS detector, JHEP 11 (2014) 118 [arXiv:1407.0583] [INSPIRE].

[78] ATLAS collaboration, Search for direct pair production of the top squark in all-hadronic final states in proton-proton collisions at $\sqrt{s}=8 \mathrm{TeV}$ with the ATLAS detector, JHEP 09 (2014) 015 [arXiv: 1406.1122] [INSPIRE].

[79] ATLAS collaboration, Search for strong production of supersymmetric particles in final states with missing transverse momentum and at least three b-jets at $\sqrt{s}=8 \mathrm{TeV}$ proton-proton collisions with the ATLAS detector, JHEP 10 (2014) 024 [arXiv:1407.0600] [INSPIRE].

[80] ATLAS collaboration, Search for direct top-squark pair production in final states with two leptons in pp collisions at $\sqrt{s}=8 \mathrm{TeV}$ with the ATLAS detector, JHEP 06 (2014) 124 [arXiv: 1403.4853] [INSPIRE].

[81] ATLAS collaboration, Search for pair-produced third-generation squarks decaying via charm quarks or in compressed supersymmetric scenarios in pp collisions at $\sqrt{s}=8 \mathrm{TeV}$ with the ATLAS detector, Phys. Rev. D 90 (2014) 052008 [arXiv: 1407.0608] [InSPIRE]. 
[82] ATLAS collaboration, Search for direct pair production of a chargino and a neutralino decaying to the $125 \mathrm{GeV}$ Higgs boson in $\sqrt{s}=8 \mathrm{TeV}$ pp collisions with the ATLAS detector, Eur. Phys. J. C $\mathbf{7 5}$ (2015) 208 [arXiv:1501.07110] [InSPIRE].

[83] ATLAS collaboration, Search for Scalar Charm Quark Pair Production in pp Collisions at $\sqrt{s}=8 \mathrm{TeV}$ with the ATLAS Detector, Phys. Rev. Lett. 114 (2015) 161801 [arXiv: 1501.01325] [INSPIRE].

[84] CMS collaboration, Search for heavy stable charged particles with $12.9 \mathrm{fb}^{-1}$ of 2016 data, Tech. Rep. CMS-PAS-EXO-16-036, CERN, Geneva (2016).

[85] CMS collaboration, Search for SUSY with multileptons in 13 TeV data, Tech. Rep. CMS-PAS-SUS-16-022, CERN, Geneva (2016).

[86] CMS collaboration, Search for supersymmetry in events with at least one soft lepton, low jet multiplicity, and missing transverse momentum in proton-proton collisions at $\sqrt{s}=13 \mathrm{TeV}$, Tech. Rep. CMS-PAS-SUS-16-052, CERN, Geneva (2017).

[87] CMS collaboration, Combined search for electroweak production of charginos and neutralinos in pp collisions at $\sqrt{s}=13 \mathrm{TeV}$, Tech. Rep. CMS-PAS-SUS-17-004, CERN, Geneva (2017).

[88] CMS collaboration, Search for the pair production of third-generation squarks with two-body decays to a bottom or charm quark and a neutralino in proton-proton collisions at $\sqrt{s}=13$ TeV, Phys. Lett. B 778 (2018) 263 [arXiv:1707.07274] [INSPIRE].

[89] CMS collaboration, Search for supersymmetry in multijet events with missing transverse momentum in proton-proton collisions at 13 TeV, Phys. Rev. D 96 (2017) 032003 [arXiv: 1704.07781] [INSPIRE].

[90] A. Nakamula, S. Sasaki and K. Takesue, Atiyah-Manton Construction of Skyrmions in Eight Dimensions, JHEP 03 (2017) 076 [arXiv: 1612.06957] [rnSPIRE].

[91] CMS collaboration, Search for physics beyond the standard model in events with two leptons of same sign, missing transverse momentum, and jets in proton-proton collisions at $\sqrt{s}=13 \mathrm{TeV}$, Eur. Phys. J. C 77 (2017) 578 [arXiv:1704.07323] [INSPIRE].

[92] CMS collaboration, Search for new phenomena with the $M_{\mathrm{T} 2}$ variable in the all-hadronic final state produced in proton-proton collisions at $\sqrt{s}=13$ TeV, Eur. Phys. J. C 77 (2017) 710 [arXiv: 1705.04650] [INSPIRE].

[93] CMS collaboration, Search for Supersymmetry in pp Collisions at $\sqrt{s}=13 \mathrm{TeV}$ in the Single-Lepton Final State Using the Sum of Masses of Large-Radius Jets, Phys. Rev. Lett. 119 (2017) 151802 [arXiv:1705.04673] [INSPIRE].

[94] S. Grozdanov and A.O. Starinets, Second-order transport, quasinormal modes and zero-viscosity limit in the Gauss-Bonnet holographic fluid, JHEP 03 (2017) 166 [arXiv: 1611.07053] [INSPIRE].

[95] W. Song, Q. Wen and J. Xu, Modifications to Holographic Entanglement Entropy in Warped CFT, JHEP 02 (2017) 067 [arXiv: 1610.00727] [INSPIRE].

[96] CMS collaboration, Search for supersymmetry in events with one lepton and multiple jets exploiting the angular correlation between the lepton and the missing transverse momentum in proton-proton collisions at $\sqrt{s}=13 \mathrm{TeV}$, Phys. Lett. B 780 (2018) 384

[arXiv: 1709.09814] [INSPIRE]. 
[97] CMS collaboration, Search for electroweak production of charginos and neutralinos in WH events in proton-proton collisions at $\sqrt{s}=13 \mathrm{TeV}$, JHEP 11 (2017) 029 [arXiv: 1706. 09933] [INSPIRE].

[98] CMS collaboration, Search for supersymmetry with Higgs boson to diphoton decays using the razor variables at $\sqrt{s}=13 \mathrm{TeV}$, Phys. Lett. B 779 (2018) 166 [arXiv:1709.00384] [INSPIRE].

[99] CMS collaboration, Search for gauge-mediated supersymmetry in events with at least one photon and missing transverse momentum in pp collisions at $\sqrt{s}=13 \mathrm{TeV}$, Phys. Lett. B 780 (2018) 118 [arXiv:1711.08008] [INSPIRE].

[100] CMS collaboration, Search for supersymmetry in events with at least one photon, missing transverse momentum, and large transverse event activity in proton-proton collisions at $\sqrt{s}=13 \mathrm{TeV}$, JHEP 12 (2017) 142 [arXiv:1707.06193] [INSPIRE].

[101] CMS collaboration, Search for direct production of supersymmetric partners of the top quark in the all-jets final state in proton-proton collisions at $\sqrt{s}=13 \mathrm{TeV}, J H E P 10$ (2017) 005 [arXiv: 1707.03316] [INSPIRE].

[102] CMS collaboration, Search for supersymmetry in proton-proton collisions at $13 \mathrm{TeV}$ using identified top quarks, Phys. Rev. D 97 (2018) 012007 [arXiv:1710.11188] [InSPIRE].

[103] CMS collaboration, Search for top squark pair production in pp collisions at $\sqrt{s}=13 \mathrm{TeV}$ using single lepton events, JHEP 10 (2017) 019 [arXiv: 1706.04402] [INSPIRE].

[104] CMS collaboration, Search for top squarks and dark matter particles in opposite-charge dilepton final states at $\sqrt{s}=13 \mathrm{TeV}$, Phys. Rev. D 97 (2018) 032009 [arXiv:1711.00752] [INSPIRE].

[105] CMS collaboration, Searches for Long-Lived Charged Particles in pp Collisions at $\sqrt{s}=7$ and $8 \mathrm{TeV}$, JHEP 07 (2013) 122 [arXiv:1305.0491] [INSPIRE].

[106] CMS collaboration, Constraints on the pMSSM, AMSB model and on other models from the search for long-lived charged particles in proton-proton collisions at $\operatorname{sqrt}(s)=8 \mathrm{TeV}$, Eur. Phys. J. C $\mathbf{7 5}$ (2015) 325 [arXiv: 1502 .02522] [INSPIRE].

[107] CMS collaboration, Search for top squarks in multijet events with large missing momentum in proton-proton collisions at $8 \mathrm{TeV}$, Tech. Rep. CMS-PAS-SUS-13-015, CERN, Geneva (2013).

[108] CMS collaboration, Search for supersymmetry in pp collisions at $\sqrt{s}=8 \mathrm{TeV}$ in events with two opposite sign leptons, large number of jets, b-tagged jets, and large missing transverse energy, Tech. Rep. CMS-PAS-SUS-13-016, CERN, Geneva (2013).

[109] CMS collaboration, Search for direct production of bottom squark pairs, Tech. Rep. CMS-PAS-SUS-13-018, CERN, Geneva (2014).

[110] CMS collaboration, A Search for Scalar Top Quark Production and Decay to All Hadronic Final States in pp Collisions at $\sqrt{s}=8 \mathrm{TeV}$, Tech. Rep. CMS-PAS-SUS-13-023, CERN, Geneva (2015).

[111] CMS collaboration, Search for Gluino Mediated Bottom- and Top-Squark Production in Multijet Final States in pp Collisions at 8 TeV, Phys. Lett. B 725 (2013) 243 [arXiv:1305.2390] [INSPIRE].

[112] CMS collaboration, Search for Supersymmetry in Hadronic Final States with Missing Transverse Energy Using the Variables $\alpha_{T}$ and b-Quark Multiplicity in pp collisions at $\sqrt{s}=8 \mathrm{TeV}$, Eur. Phys. J. C 73 (2013) 2568 [arXiv:1303.2985] [INSPIRE]. 
[113] CMS collaboration, Search for anomalous production of events with three or more leptons in pp collisions at $\sqrt{s}=8 \mathrm{TeV}$, Phys. Rev. D 90 (2014) 032006 [arXiv:1404.5801] [INSPIRE].

[114] CMS collaboration, Search for Supersymmetry Using Razor Variables in Events with b-Tagged Jets in pp Collisions at $\sqrt{s}=8$ TeV, Phys. Rev. D 91 (2015) 052018 [arXiv: 1502.00300] [INSPIRE].

[115] CMS collaboration, Searches for electroweak production of charginos, neutralinos, and sleptons decaying to leptons and W, Z, and Higgs bosons in pp collisions at 8 TeV, Eur. Phys. J. C 74 (2014) 3036 [arXiv:1405.7570] [InSPIRE].

[116] CMS collaboration, Search for Supersymmetry in pp collisions at $8 \mathrm{TeV}$ in events with a single lepton, multiple jets and b-tags, Tech. Rep. CMS-PAS-SUS-13-007, CERN, Geneva (Mar, 2013).

[117] CMS collaboration, Search for Top-Squark Pair Production in the Single-Lepton Final State in pp Collisions at $\sqrt{s}=8 \mathrm{TeV}$, Eur. Phys. J. C 73 (2013) 2677 [arXiv:1308.1586] [INSPIRE].

[118] CMS collaboration, Search for new physics in the multijet and missing transverse momentum final state in proton-proton collisions at $\sqrt{s}=8 \mathrm{TeV}$, JHEP 06 (2014) 055 [arXiv:1402.4770] [INSPIRE].

[119] P. Schweitzer, M. Strikman and C. Weiss, Intrinsic transverse momentum and parton correlations from dynamical chiral symmetry breaking, JHEP 01 (2013) 163 [arXiv: 1210.1267] [INSPIRE].

[120] CMS collaboration, Searches for Supersymmetry using the $M_{T 2}$ Variable in Hadronic Events Produced in pp Collisions at 8 TeV, JHEP 05 (2015) 078 [arXiv:1502.04358] [INSPIRE].

[121] CMS collaboration, Searches for supersymmetry based on events with b jets and four $W$ bosons in pp collisions at $8 \mathrm{TeV}$, Phys. Lett. B 745 (2015) 5 [arXiv:1412.4109] [InSPIRE].

[122] CMS collaboration, Search for supersymmetry in events with soft leptons, low jet multiplicity, and missing transverse energy in proton-proton collisions at $\sqrt{s}=8$ TeV, Phys. Lett. B 759 (2016) 9 [arXiv:1512.08002] [INSPIRE].

[123] T. Sjöstrand, S. Mrenna and P.Z. Skands, PYTHIA 6.4 Physics and Manual, JHEP 05 (2006) 026 [hep-ph/0603175] [INSPIRE].

[124] T. Sjöstrand et al., An introduction to PYTHIA 8.2, Comput. Phys. Commun. 191 (2015) 159 [arXiv: 1410.3012] [INSPIRE].

[125] A. Djouadi, M.M. Muhlleitner and M. Spira, Decays of supersymmetric particles: The Program SUSY-HIT (SUspect-SdecaY-HDECAY-InTerface), Acta Phys. Polon. B 38 (2007) 635 [hep-ph/0609292] [INSPIRE].

[126] W. Beenakker, R. Hopker, M. Spira and P.M. Zerwas, Squark and gluino production at hadron colliders, Nucl. Phys. B 492 (1997) 51 [hep-ph/9610490] [InSPIRE].

[127] W. Beenakker, M. Krämer, T. Plehn, M. Spira and P.M. Zerwas, Stop production at hadron colliders, Nucl. Phys. B 515 (1998) 3 [hep-ph/9710451] [InSPIRE].

[128] A. Kulesza and L. Motyka, Threshold resummation for squark-antisquark and gluino-pair production at the LHC, Phys. Rev. Lett. 102 (2009) 111802 [arXiv:0807.2405] [INSPIRE].

[129] A. Kulesza and L. Motyka, Soft gluon resummation for the production of gluino-gluino and squark-antisquark pairs at the LHC, Phys. Rev. D 80 (2009) 095004 [arXiv:0905.4749] [INSPIRE]. 
[130] W. Beenakker, S. Brensing, M. Krämer, A. Kulesza, E. Laenen and I. Niessen, Soft-gluon resummation for squark and gluino hadroproduction, JHEP 12 (2009) 041 [arXiv: 0909.4418] [INSPIRE].

[131] W. Beenakker, S. Brensing, M. Krämer, A. Kulesza, E. Laenen and I. Niessen, Supersymmetric top and bottom squark production at hadron colliders, JHEP 08 (2010) 098 [arXiv: 1006.4771] [INSPIRE].

[132] W. Beenakker et al., Squark and Gluino Hadroproduction, Int. J. Mod. Phys. A 26 (2011) 2637 [arXiv: 1105.1110] [inSPIRE].

[133] ATLAS collaboration, Summary of the ATLAS experiment's sensitivity to supersymmetry after LHC Run 1 - interpreted in the phenomenological MSSM, JHEP 10 (2015) 134 [arXiv: 1508.06608] [INSPIRE].

[134] Planck collaboration, Planck 2018 results. VI. Cosmological parameters, Astron. Astrophys. 641 (2020) A6 [arXiv:1807.06209] [INSPIRE].

[135] R.V. Harlander, S. Liebler and H. Mantler, SusHi Bento: Beyond NNLO and the heavy-top limit, Comput. Phys. Commun. 212 (2017) 239 [arXiv:1605.03190] [InSPIRE].

[136] http://suspect.in2p3.fr/.

[137] DELPHES 3 collaboration, DELPHES 3, A modular framework for fast simulation of a generic collider experiment, JHEP 02 (2014) 057 [arXiv:1307.6346] [INSPIRE].

[138] D. Binosi, J. Collins, C. Kaufhold and L. Theussl, JaxoDraw: A graphical user interface for drawing Feynman diagrams. Version 2.0 release notes, Comput. Phys. Commun. 180 (2009) 1709 [arXiv:0811.4113] [INSPIRE].

[139] CRESST collaboration, Results on light dark matter particles with a low-threshold CRESST-II detector, Eur. Phys. J. C 76 (2016) 25 [arXiv:1509.01515] [InSPIRE].

[140] S. Dittmaier, M. Krämer and M. Spira, Higgs radiation off bottom quarks at the Tevatron and the CERN LHC, Phys. Rev. D 70 (2004) 074010 [hep-ph/0309204] [InSPIRE].

[141] S. Dawson, C.B. Jackson, L. Reina and D. Wackeroth, Higgs production in association with bottom quarks at hadron colliders, Mod. Phys. Lett. A 21 (2006) 89 [hep-ph/0508293] [INSPIRE].

[142] S. Dawson, C.B. Jackson, L. Reina and D. Wackeroth, Higgs boson production with bottom quarks at hadron colliders, Int. J. Mod. Phys. A 20 (2005) 3353 [hep-ph/0409345] [INSPIRE].

[143] S. Dawson, C.B. Jackson, L. Reina and D. Wackeroth, Exclusive Higgs boson production with bottom quarks at hadron colliders, Phys. Rev. D 69 (2004) 074027 [hep-ph/0311067] [INSPIRE].

[144] R.V. Harlander and W.B. Kilgore, Higgs boson production in bottom quark fusion at next-to-next-to leading order, Phys. Rev. D 68 (2003) 013001 [hep-ph/0304035] [INSPIRE].

[145] S. Dawson, C.B. Jackson, L. Reina and D. Wackeroth, Higgs boson production with one bottom quark jet at hadron colliders, Phys. Rev. Lett. 94 (2005) 031802 [hep-ph/0408077] [INSPIRE].

[146] S. Dawson and P. Jaiswal, Weak Corrections to Associated Higgs-Bottom Quark Production, Phys. Rev. D 81 (2010) 073008 [arXiv:1002.2672] [INSPIRE].

[147] F. Maltoni, Z. Sullivan and S. Willenbrock, Higgs-Boson Production via Bottom-Quark Fusion, Phys. Rev. D 67 (2003) 093005 [hep-ph/0301033] [INSPIRE]. 
[148] E. Boos and T. Plehn, Higgs boson production induced by bottom quarks, Phys. Rev. D 69 (2004) 094005 [hep-ph/0304034] [INSPIRE].

[149] T. Plehn, Charged Higgs boson production in bottom gluon fusion, Phys. Rev. D 67 (2003) 014018 [hep-ph/0206121] [INSPIRE].

[150] R. Harlander, M. Krämer and M. Schumacher, Bottom-quark associated Higgs-boson production: reconciling the four- and five-flavour scheme approach, arXiv:1112.3478 [INSPIRE].

[151] J. Alwall et al., The automated computation of tree-level and next-to-leading order differential cross sections, and their matching to parton shower simulations, JHEP $\mathbf{0 7}$ (2014) 079 [arXiv: 1405.0301] [InSPIRE].

[152] CMS collaboration, Identification of heavy-flavour jets with the CMS detector in $p p$ collisions at $13 \mathrm{TeV}, 2018$ JINST 13 P05011 [arXiv:1712.07158] [INSPIRE].

[153] M. Cacciari, G.P. Salam and G. Soyez, FastJet User Manual, Eur. Phys. J. C 72 (2012) 1896 [arXiv:1111.6097] [INSPIRE].

[154] W. Beenakker, R. Hopker and M. Spira, PROSPINO: A program for the production of supersymmetric particles in next-to-leading order QCD, hep-ph/9611232 [INSPIRE].

[155] M. Wiesemann, R. Frederix, S. Frixione, V. Hirschi, F. Maltoni and P. Torrielli, Higgs production in association with bottom quarks, JHEP 02 (2015) 132 [arXiv:1409.5301] [INSPIRE].

[156] M.L. Mangano, M. Moretti, F. Piccinini and M. Treccani, Matching matrix elements and shower evolution for top-quark production in hadronic collisions, JHEP 01 (2007) 013 [hep-ph/0611129] [INSPIRE].

[157] A. Lazopoulos, T. McElmurry, K. Melnikov and F. Petriello, Next-to-leading order QCD

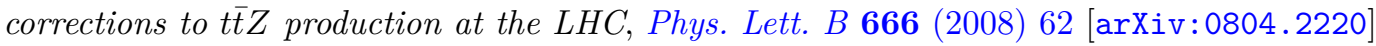
[INSPIRE].

[158] https://twiki.cern.ch/twiki/bin/view/LHCPhysics/TtbarNNLO.

[159] M. Bonvini, A.S. Papanastasiou and F.J. Tackmann, Matched predictions for the $b \bar{b} H$ cross section at the $13 \mathrm{TeV} L H C$, JHEP 10 (2016) 053 [arXiv:1605.01733] [INSPIRE].

[160] A. Adhikary, S. Banerjee, R.K. Barman, B. Bhattacherjee and S. Niyogi, Revisiting the non-resonant Higgs pair production at the HL-LHC, JHEP 07 (2018) 116 [arXiv: 1712.05346] [INSPIRE].

[161] https://twiki.cern.ch/twiki/bin/view/LHCPhysics/CERNYellowReportPageAt1314TeV2014.

[162] J.M. Campbell and R.K. Ellis, $t \bar{t} W^{+-}$production and decay at NLO, JHEP 07 (2012) 052 [arXiv: 1204.5678] [INSPIRE].

[163] ATLAS collaboration, Search for long-lived charginos based on a disappearing-track signature in pp collisions at $\sqrt{s}=13 \mathrm{TeV}$ with the ATLAS detector, JHEP 06 (2018) 022 [arXiv: 1712.02118] [INSPIRE].

[164] CMS collaboration, Search for disappearing tracks as a signature of new long-lived particles in proton-proton collisions at $\sqrt{s}=13 \mathrm{TeV}, \mathrm{JHEP} 08$ (2018) 016 [arXiv:1804.07321] [INSPIRE].

[165] ATLAS collaboration, Search for charged Higgs bosons decaying via $H^{ \pm} \rightarrow \tau^{ \pm} \nu$ in fully hadronic final states using pp collision data at $\sqrt{s}=8 \mathrm{TeV}$ with the ATLAS detector, JHEP 03 (2015) 088 [arXiv: 1412.6663] [INSPIRE]. 
[166] CMS collaboration, Search for a charged Higgs boson in pp collisions at $\sqrt{s}=8 \mathrm{TeV}$, JHEP 11 (2015) 018 [arXiv: 1508.07774] [INSPIRE].

[167] CMS collaboration, Search for charged Higgs bosons with the $\mathrm{H}^{ \pm} \rightarrow \tau^{ \pm} \nu_{\tau}$ decay channel in the fully hadronic final state at $\sqrt{s}=13 \mathrm{TeV}$, Tech. Rep. CMS-PAS-HIG-16-031, CERN, Geneva (2016).

[168] ATLAS collaboration, Search for charged Higgs bosons produced in association with a top quark and decaying via $H^{ \pm} \rightarrow \tau \nu$ using pp collision data recorded at $\sqrt{s}=13 \mathrm{TeV}$ by the ATLAS detector, Phys. Lett. B 759 (2016) 555 [arXiv: 1603.09203] [INSPIRE].

[169] ATLAS collaboration, Search for charged Higgs bosons in the $H^{ \pm} \rightarrow t b$ decay channel in $p p$ collisions at $\sqrt{s}=8 \mathrm{TeV}$ using the ATLAS detector, JHEP 03 (2016) 127 [arXiv: 1512.03704] [INSPIRE].

[170] ATLAS collaboration, Search for charged Higgs bosons in the $H^{ \pm} \rightarrow$ tb decay channel in pp collisions at $\sqrt{s}=13 \mathrm{TeV}$ using the ATLAS detector, Tech. Rep. ATLAS-CONF-2016-089, CERN, Geneva (Aug, 2016).

[171] https://cp3.irmp.ucl.ac.be/projects/madgraph/wiki/chargedHiggs\#no1.

[172] C. Degrande, M. Ubiali, M. Wiesemann and M. Zaro, Heavy charged Higgs boson production at the LHC, JHEP 10 (2015) 145 [arXiv:1507.02549] [INSPIRE].

[173] A.V. Bednyakov, B.A. Kniehl, A.F. Pikelner and O.L. Veretin, On the b-quark running mass in QCD and the SM, Nucl. Phys. B 916 (2017) 463 [arXiv:1612.00660] [InSPIRE].

[174] H. Fukuda, N. Nagata, H. Oide, H. Otono and S. Shirai, Cornering Higgsinos Using Soft Displaced Tracks, Phys. Rev. Lett. 124 (2020) 101801 [arXiv:1910.08065] [INSPIRE].

[175] H. Fukuda, N. Nagata, H. Otono and S. Shirai, Higgsino Dark Matter or Not: Role of Disappearing Track Searches at the LHC and Future Colliders, Phys. Lett. B 781 (2018) 306 [arXiv: 1703.09675] [INSPIRE].

[176] B. Bhattacherjee, B. Feldstein, M. Ibe, S. Matsumoto and T.T. Yanagida, Pure gravity mediation of supersymmetry breaking at the Large Hadron Collider, Phys. Rev. D 87 (2013) 015028 [arXiv: 1207.5453] [INSPIRE].

[177] S. Banerjee, B. Bhattacherjee, A. Goudelis, B. Herrmann, D. Sengupta and R. Sengupta, Determining the lifetime of long-lived particles at the HL-LHC, Eur. Phys. J. C $\mathbf{8 1}$ (2021) 172 [arXiv: 1912.06669] [INSPIRE]. 